Bull. Soc. math. France

133 (2), 2005, p. 205-257

\title{
THÉORIE DE VORONOÏ GÉOMÉTRIQUE. PROPRIÉTÉS DE FINITUDE POUR LES FAMILLES DE RÉSEAUX ET ANALOGUES
}

\author{
par Christophe Bavard
}

RÉSumÉ. - Nous développons une théorie de Voronoï géométrique. En l'appliquant aux familles classiques de réseaux euclidiens (par exemple symplectiques ou orthogonaux), nous obtenons notamment de nouveaux résultats de finitude concernant les configurations de vecteurs minimaux et les réseaux particuliers (par exemple parfaits) de ces familles. Les méthodes géométriques introduites sont également illustrées par l'étude d'objets voisins (formes de Humbert) ou analogues (surfaces de Riemann).

ABSTRACT (Voronoï's geometric theory. Finiteness properties for families of lattices and similar objects)

A geometric Voronoï's theory is developed and applied to classical families of euclidean lattices (such as symplectic or orthogonal lattices). In particular, new finiteness results are obtained concerning configurations of minimal vectors and special lattices (for example the perfect ones) in these families. The geometric methods introduced are also illustrated by the study of related objects (Humbert forms) or similar ones (Riemann surfaces).

Texte reçu le 21 mars 2003, accepté le 22 octobre 2003

Christophe Bavard, Laboratoire Bordelais d'Analyse et Géométrie, U.M.R. 5467 C.N.R.S., Université Bordeaux-I, 351, cours de la Libération, F-33405 Talence Cedex (France)

E-mail : Christophe.Bavard@math.u-bordeaux.fr

Classification mathématique par sujets (2000). — 11H55.

Mots clefs. - Théorie de Voronoï, réseaux euclidiens. 


\section{Introduction}

Nous nous intéressons ici à deux aspects traditionnels de la théorie des réseaux euclidiens : premièrement les réseaux particuliers liés à des propriétés variationnelles d'invariants classiques (réseaux extrêmes, parfaits, ... ), et deuxièmement les configurations de vecteurs minimaux. L'idée de considérer ces objets s'est imposée naturellement, en particulier dans l'étude de la constante d'Hermite. On sait par exemple depuis Voronoï [37] que la norme des réseaux euclidiens de déterminant 1 n'admet qu'un nombre fini de maxima locaux. De même, l'utilisation des configurations de vecteurs minimaux, formulées en termes de formes quadratiques, est assez ancienne; on la retrouve notamment dans [27] (tables d'entiers représentant le minimum). Les résultats de finitude concernant ces réseaux particuliers et ces configurations sont préliminaires à toute classification. Ils jouent donc un rôle fondamental, aussi bien dans la théorie classique que dans ses extensions récentes (voir [7, 9, 10, 11, 26, 28]).

Les concepts précédents, issus de la théorie des réseaux, ont été définis dans le contexte général et entièrement géométrique des systoles généralisées de [5]. Nous proposons ici de nouveaux développements de ce point de vue : introduction de la notion de point non dégénéré, étude systématique des points particuliers, des configurations et de leurs relations mutuelles (première partie de l'article). Dans le cadre unificateur de cette théorie de Voronoï géométrique, les principaux résultats de finitude (relatifs à ces notions) connus pour les réseaux découlent essentiellement d'un petit nombre de propriétés géométriques ou topologiques élémentaires des espaces de paramètres. L'application aux familles de réseaux de ces techniques occupe une grande part de la seconde partie. Elle s'effectue en rapprochant description géométrique et étude algébrique. Ainsi, nous obtenons d'abord de nouveaux résultats de finitude, concernant notamment les réseaux autoduaux, par exemple orthogonaux ou symplectiques ; parallèlement, afin de compléter les propriétés de finitude et en vue de futures classifications, nous mettons en évidence des contraintes algébriques satisfaites par les réseaux autoduaux particuliers. De nouveaux résultats sont également établis dans le cadre géométrique pour les invariants de Bergé-Martinet et d'HermiteHumbert attaché à un corps de nombre. Enfin, la portée de nos méthodes est aussi illustrée dans un contexte différent, celui des surfaces de Riemann. Voici maintenant quelques commentaires plus détaillés sur le contenu de l'article.

Les notions générales de perfection et d'eutaxie sont rappelées au $\S 1$.1. Le cadre de [5] a été sensiblement élargi : on travaille avec une variété lisse $V$ munie d'une connexion et de fonctions longueur $f_{s}$ convexoïdales (définition 1.4), mais pas forcément convexes. Cette propriété a l'avantage d'être invariante par changement de paramétrage et composition au but par un $\mathcal{C}^{1}$-difféomorphisme 
croissant (voir son intérêt aux $\S \S 2.3$ et 2.4). Les configurations minimales interviennent via les classes minimales, terminologie introduite dans [8] pour les réseaux. La classe minimale associée à une configuration donnée $S$ est l'ensemble des points de $V$ qui admettent $\left(f_{s}\right)_{s \in S}$ comme famille de fonctions minimales.

Le cadre de [5] a aussi été enrichi. Nous introduisons tout d'abord la notion de point non dégénéré (définition 1.5) ; par exemple un réseau dont les vecteurs minimaux engendrent l'espace est non dégénéré relativement à toute famille de réseaux. Elle permet premièrement d'établir une caractérisation du théorème de Voronoï par la non dégénérescence des points extrêmes (proposition 1.5) qui clarifie l'approche de [10] pour le cas particulier des réseaux orthogonaux ou symplectiques. On ne donnait dans [5] qu'une condition suffisante assurant le théorème de Voronoï, la «condition $(C)$ ». Deuxièmement, les points eutactiques non dégénérés constituent une généralisation satisfaisante des formes eutactiques de la théorie usuelle. Ainsi pour les familles de réseaux autoduaux $(\S 2.7)$, en particulier orthogonaux ou symplectiques, ces points ont de très bonnes propriétés : finitude (théorème 1, 2c), algébricité (corollaire 1.12), rang maximal des vecteurs minimaux dans le cas symplectique (corollaire 2.15). Bien entendu, ces propriétés sont généralement fausses pour les points eutactiques, même dans les meilleures situations (réseaux symplectiques par exemple). Ensuite, les relations entre perfection, eutaxie et classes minimales sont systématiquement explorées : caractérisation variationnelle des points eutactiques dans une classe (proposition 1.6), propriétés d'isolement des points parfaits ou eutactiques dans une classe (proposition 1.8 et 1.9) en vue de résultats de finitude et d'algébricité (lemme 1.11 et corollaire 1.12).

Les problèmes de finitude dans $V$ se posent toujours modulo l'action d'un certain groupe discret naturel de difféomorphismes $\Gamma$ qui préserve toutes les structures en jeu (géométriques et algébriques). Typiquement, $V$ est un espace «d'objets marqués » (espace de formes quadratiques, espace de Teichmüller,...) tandis que $\Gamma \backslash V$ paramètre les «modules» de ces objets (réseaux, surfaces de Riemann,...). Pour traiter les questions de finitude nous procédons selon le schéma général suivant. Nous cherchons tout d'abord un théorème de compacité de type Mahler dans l'espace des modules $\Gamma \backslash V$. Pour les familles de réseaux, ces propriétés de compacité traduisent ou remplacent très souvent des arguments de nature combinatoire. Nous établissons ensuite un résultat de finitude pour un certain type de classes minimales liées à la nature des objets étudiés et qui restent loin de l'infini dans $\Gamma \backslash V$, par exemple les classes «non isotropes » pour les réseaux autoduaux (proposition 2.13) ou pour les surfaces de Riemann ( $\S 2.12)$. Puis nous montrons que les points particuliers (parfaits,...), grâce à leurs propriétés algébriques, appartiennent aux classes en question (exemple : théorème 1,1). Il reste enfin à établir qu'ils sont en nombre fini dans chaque classe minimale, soit par un argument 
variationnel pour les points eutactiques (proposition 1.6), soit par un lemme de géométrie algébrique (lemme 1.11) pour les points parfaits.

Cette démarche est mise en œuvre dans la deuxième partie de l'article pour les familles de réseaux, que nous paramétrons par des sous-variétés totalement géodésiques et algébriques $V$ de l'espace symétrique riemannien $P_{n}^{\mathbb{R}}$ des formes quadratiques. Dans les situations naturelles, $V$ apparait aussi comme une orbite d'un groupe de Lie réel $G(\mathbb{R})$. Les propriétés de compacité de $G(\mathbb{Z}) \backslash G(\mathbb{R})$ pour certains groupes algébriques complexes $G$ (par exemple réductifs) sont classiques dans l'étude des groupes arithmétiques. Des techniques correspondantes, nous avons extrait une condition élémentaire et facilement vérifiable (la «pseudo-algébricité») qui assure la compacité dans les familles de réseaux (proposition 2.1).

Les familles de réseaux les plus simples sont les réseaux usuels muni de l'action d'un groupe fini (П-réseaux). Leur comportement est totalement analogue au cas usuel où $\Pi$ est trivial (voir $[9,12,26,11]$ ). Munies d'une carte affine globale et d'une connexion naturelle $(\S 2.3)$, elles ont des propriétés géométriques très fortes, par exemple les classes minimales sont géodésiques; nous donnons ainsi une description précise de ce cas (proposition 2.5) qui contient les résultats de finitude connus (voir corollaire 2.6 et note 2.5 ). Plus généralement il est important de considérer une famille quelconque $V$ munie d'une action $\rho$ d'un groupe fini préservant la caractérisation, en général algébrique, des éléments de $V$. Cette situation est décrite par le lieu des points fixes $V^{\rho}$ dont les propriétés (géométriques, algébriques et topologiques) sont établies à la proposition 2.7. L'étude pratique de $V^{\rho}$ quand il est de petite dimension permet souvent de mettre en évidence des réseaux intéressants; c'est une source importante d'exemples (voir [6] pour le cas symplectique). Dans le même esprit, l'eutaxie relativement à $V$ se lit dans $V^{\rho}$ (lemme 2.8). Le contexte équivariant permet également de donner une interprétation symplectique de l'invariant de Bergé-Martinet (§ 2.10).

Nous proposons ensuite une étude unifiée des autres familles naturelles de réseaux (orthogonaux, symplectiques,... ) sous le terme de réseaux autoduaux pour une forme bilinéaire ou sesquilinéaire $b$ (§ 2.7). Comme les réseaux hermitiens complexes et quaternioniens correspondent aussi à un espace symétrique $P_{m}^{k}(\boldsymbol{k}=\mathbb{C}$ ou $\mathbb{H})$ muni de longueurs analogues au cas réel $(\S 2.6)$, il n'y avait pas de raison de se limiter à ce cas. D'autre part, compte tenu des motivations évoquées plus haut, nous nous sommes placés dans le contexte équivariant. À type algébrique fixé (définition 2.2), les réseaux $b$-autoduaux sont paramétrés par un espace symétrique (proposition 2.9, exemple 2.11) avec de bonnes propriétés algébriques et topologiques (proposition 2.11 et corollaire 2.12). Nous exploitons systématiquement la structure algébrique définie par $b$. Ainsi, une fois la notion centrale de classes non isotropes définie, la finitude de ces classes 
(proposition 2.13) résulte facilement de l'inégalité de Cauchy-Schwarz, qui dans notre cadre remplace avantageusement celle de Hadamard.

Dans le cas où $b$ est réflexive (§2.8), qui recouvre les principales familles classiques (par exemple associées aux espaces symétriques irréductibles, exemple 2.11), les résultats sont plus précis. Nous obtenons tout d'abord le fait que les réseaux faiblement eutactiques sont non isotropes, d'où découle la finitude des réseaux parfaits ou eutactiques non dégénérés (théorème 1). Parallèlement, en comparant les caractéristiques algébriques de $b$ (isotropie) avec celles de l'action (types), nous mettons en évidence des contraintes fortes sur les vecteurs minimaux de ces réseaux, notamment sur leur rang (théorème 2 , 3 et corollaire 2.16, 2.17). Par exemple, dans le cas symplectique, le rang des vecteurs minimaux est maximal pour un réseau parfait si l'action est isotypique et symplectique, ou pour un réseau eutactique non dégénéré si l'action est diagonalisable. Enfin, une étude algébrique des classes minimales voisines de l'infini (i.e. isotropes) permet d'établir la finitude des classes minimales dans le cas réel ou complexe sans action (théorème 4 ).

L'interprétation symplectique équivariante de l'invariant de Bergé-Martinet $\gamma_{m, 1}^{\prime}(\S 2.10)$ fournit une définition naturelle des couples parfaits de [7] (proposition 2.20) et permet, en particulier avec la finitude des classes non isotropes, de retrouver les propriétés de finitude liées à $\gamma_{m, 1}^{\prime}$ (voir note 2.18). À l'aide d'un invariant $\nu_{m, k}$ très voisin de l'invariant de Bergé-Martinet-Rankin $\gamma_{m, k}^{\prime}$ $(1 \leq k \leq m-1)$, nous établissons une caractérisation des maxima locaux de $\gamma_{m, k}^{\prime}$, répondant ainsi au problème 6.10 de $[28$, ch. X] et à son analogue complexe (théorème 5).

Une autre illustration des méthodes géométriques est donnée par l'étude des formes de Humbert associées à un corps de nombre (§2.11). Nous prouvons notamment que l'invariant d'Hermite-Humbert vérifie la condition $(C)$ (proposition 2.22), donc le théorème de Voronoï (voir [19]), et que les formes de Humbert non dégénérées restent loin de l'infini, avec comme corollaire la finitude des formes de Humbert eutactiques non dégénérées (proposition 2.24).

La systole des surfaces de Riemann est examinée au $\S 2.12$. Inspirée du cas des réseaux, la notion de surface isotrope nous permet de prouver des résultats analogues : les surfaces de Riemann parfaites ou eutactiques pour la systole sont non isotropes et en nombre fini; de plus les longueurs de leurs géodésiques fermées sont des logarithmes de nombres algébriques (théorème 6).

\section{Plan de l'article}

Introduction .......................................206

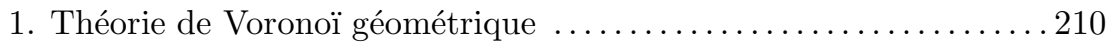

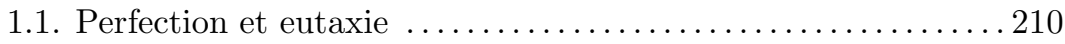

1.2. Propriétés géométriques. Points non dégénérés et théorème de

Voronoï ......................................213

BULLETIN DE LA SOCIÉTÉ MATHÉMATIQUE DE FRANCE 


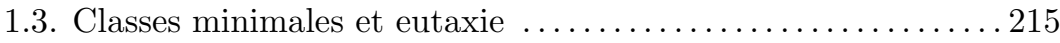

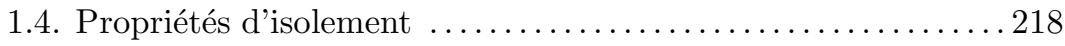

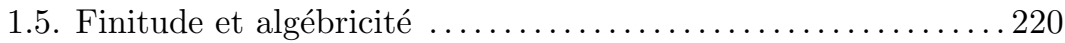

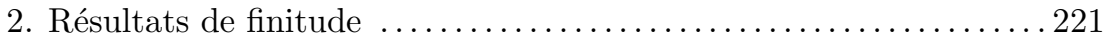

2.1. Compacité dans l'espace des réseaux . . . . . . . . . . . 221

2.2. Non dégénérescence dans les familles de réseaux . . . . . . . . . 224

2.3. Une connexion naturelle sur l'espace des formes . . . . . . . . 225

2.4. Réseaux avec action d'un groupe fini .................226

2.5. Familles de réseaux avec action d'un groupe fini . . . . . . . . 228

2.6. Réseaux hermitiens complexes et quaternioniens . . . . . . . 231

2.7. Autodualité : propriétés générales ...................233

2.8. Autodualité : le cas réflexif . . . . . . . . . . . . . . . . . 237

2.9. Finitude des classes minimales pour les réseaux autoduaux . 243

2.10. Interprétation symplectique de l'invariant de Bergé-Martinet 245

2.11. Invariant d'Hermite-Humbert . . . . . . . . . . . . . . . . . 249

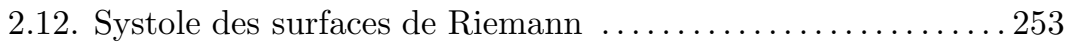

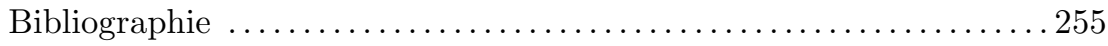

\section{Théorie de Voronoï géométrique}

1.1. Perfection et eutaxie. - Voronoï [37, p. 126] caractérise les maxima locaux de l'invariant d'Hermite par deux propriétés : perfection et eutaxie. Ces notions ont été étendues plus récemment aux familles de réseaux (voir [10]) et à l'invariant de Rankin (voir [18]), puis sous une forme géométrique et générale dans [5]. Afin de rappeler la terminologie introduite dans [5], formulons d'abord une définition dans le cadre de l'algèbre linéaire élémentaire.

DÉFInition 1.1. - Soit $E$ un $\mathbb{R}$-espace vectoriel de dimension finie et soit $\mathcal{F}$ une famille finie de vecteurs de $E$. Notons $K$ l'enveloppe convexe de $\mathcal{F}$.

1) $\mathcal{F}$ est parfaite si elle engendre affinement $E$.

2) $\mathcal{F}$ est eutactique (resp. semi-eutactique, resp. faiblement eutactique) si le vecteur nul 0 de $E$ appartient à l'intérieur affine de $K$ (resp. à $K$, resp. au sous-espace affine engendré par $K$ ).

N.B. - Notre définition ne coïncide pas avec celle de [28, III, déf. 2.2]. Nous adoptons cette terminologie qui est pratique et bien adaptée au cas général (voir notamment les lemmes 1.1, 1.2 et 1.10).

Considérons maintenant une variété lisse et connexe $V$, un ensemble $C$ et une famille de fonctions $f_{s}: V \rightarrow \mathbb{R}(s \in C)$ de classe $\mathcal{C}^{1}$. Nous dirons que $\left(f_{s}\right)_{s \in C}$ est un système de fonctions longueur indexé par $C$ (courbes) si pour tout point $p$ de $V$ et pour tout réel $L$, il existe un voisinage ouvert $U$ de $p$ tel que 
l'ensemble $C \backslash\left\{s \in C ; f_{s \mid U}>L\right\}$ soit fini. Autrement dit, localement, il n'y a qu'un nombre fini de $f_{s}$ pouvant prendre une valeur petite. On s'intéresse alors à la borne inférieure $\mu$ des $\left(f_{s}\right)_{s \in C}$, ou systole généralisée, qui est localement le minimum d'un nombre fini de $f_{s}$, donc continue:

$$
\mu(p)=\min _{s \in C} f_{s}(p) \quad(p \in V) .
$$

Remarque 1.1. - La finitude locale ci-dessus résulte très souvent de la finitude ponctuelle et d'un contrôle sur les gradients des $f_{s}$ (en supposant $V$ riemannienne). Par exemple, s'il existe $a>0$ tel que $\left\|\nabla f_{s}\right\| \leq a f_{s}$ pour tout élément $s \in C$ (c'est le cas pour les réseaux), alors pour $p \in V$ et $\operatorname{dist}(p, q) \leq r$ $\left(r>0\right.$ petit), l'inégalité $f_{s}(q) \leq L$ entraîne $f_{s}(p) \leq L+\exp (a r)$.

L'hypothèse donnée ici pour les $f_{s}$ est vérifiée pour tous les exemples naturels. Elle est légèrement plus forte que celle de [5] qui n'implique pas formellement la continuité de $\mu$ (remarque due à P. Buser) implicitement utilisée dans cet article. Posons $S_{p}=\left\{s \in C ; f_{s}(p)=\mu(p)\right\}$, ensemble des « courbes minimales » au point $p$.

DÉfinition 1.2. - Un point $p$ de $V$ est parfait (resp. eutactique, semi-eutactique, faiblement eutactique) si la famille des différentielles $\left(\mathrm{d} f_{s}(p)\right)_{s \in S_{p}}$ est parfaite (resp. eutactique, semi-eutactique, faiblement eutactique) dans l'espace cotangent $T_{p}^{*} V$.

D'après [5] et proposition $2.22,1$ ), cette définition généralise la terminologie classique ainsi que ses extensions récentes dans la théorie des réseaux (voir $[10,18,19])$.

Remarques 1.2. - 1) Quand $V$ est munie d'une métrique riemannienne, perfection et eutaxie s'expriment naturellement avec les gradients $\nabla f_{s}(p)$ dans l'espace tangent $T_{p} V$.

2) La nature des points de $V$ (perfection,...) est inchangée si on compose toutes les $f_{s}$ par un même $\mathcal{C}^{1}$-difféomorphisme croissant.

DÉfinition 1.3. - Soit $(E,\langle.,\rangle$.$) un \mathbb{R}$-espace vectoriel euclidien de dimension finie et soit $\mathcal{F}=\left(X_{s}\right)_{s \in S}$ une famille finie de vecteurs de $E$. Soit $X \in E$.

1) $X$ est positif (relativement à $\mathcal{F}$ ) si $\left\langle X, X_{s}\right\rangle>0$ pour tout $s \in S$.

2) $X$ est semi-positif si $\left\langle X, X_{s}\right\rangle \geq 0$ pour tout $s \in S$ et $\sum_{s \in S}\left\langle X, X_{s}\right\rangle=1$.

3) $X$ est faiblement positif si $\left\langle X, X_{s}\right\rangle \geq 0$ pour tout $s \in S$.

L'existence d'un vecteur positif (resp. semi-positif, faiblement positif) relativement à $\mathcal{F}$ est évidemment indépendante du produit scalaire sur $E$. Le lemme élémentaire suivant permet de caractériser par dualité perfection et eutaxie dans un espace vectoriel.

BULlETIN DE LA SOCIÉTÉ MATHÉMATIQUE DE FRANCE 
Lemme 1.1. - Soient $E$ et $\mathcal{F}$ comme dans la définition 1.3.

1) $\mathcal{F}$ est parfaite et eutactique si et seulement s'il n'existe pas de vecteur faiblement positif (relativement à $\mathcal{F}$ ) non nul.

2) $\mathcal{F}$ est eutactique si et seulement s'il n'existe pas de vecteur semi-positif.

3) $\mathcal{F}$ est semi-eutactique si et seulement s'il n'existe pas de vecteur positif.

4) $\mathcal{F}$ est faiblement eutactique si et seulement si tout $X$ tel que $\left\langle X, X_{s}\right\rangle$ ne dépend pas de $s \in S$ est orthogonal à $\mathcal{F}$.

Preuve. - Soit $K$ l'enveloppe convexe de $\mathcal{F}$.

2) Si $\mathcal{F}$ satisfait une relation d'eutaxie, il ne peut exister de vecteur semipositif. Si $\mathcal{F}$ n'est pas eutactique, on obtient par séparation un vecteur positif ou semi-positif, suivant que $0 \notin K$ ou que 0 appartient à une face stricte de $K$.

$1)$ Si $\mathcal{F}$ est parfaite et eutactique, alors $\mathcal{F}$ engendre vectoriellement $E$. Tout vecteur faiblement positif est orthogonal à $\mathcal{F}$ par 2), donc nul. Si $\mathcal{F}$ n'est pas eutactique, il existe un vecteur semi-positif par 2). Si $\mathcal{F}$ est eutactique non parfaite, il existe un vecteur non nul orthogonal à $\mathcal{F}$.

Les assertions 3) et 4) sont encore plus évidentes que 1) et 2).

Remarquons enfin que si un groupe fini agit sur la situation, les propriétés d'eutaxie se lisent sur le sous-espace des points fixes.

Lemme 1.2. - Soient $E$ et $\mathcal{F}$ comme dans la définition 1.3 , et soit $\Pi$ un groupe fini d'isométries de $E$ qui laisse stable $\mathcal{F}$. On note $F^{\Pi}$ le sous-espace de $E$ des vecteurs fixes de $\Pi$ et $p$ la projection orthogonale sur $F^{\Pi}$. Alors $\mathcal{F}=\left(X_{s}\right)_{s \in S}$ est eutactique (resp. semi-eutactique, faiblement eutactique) si et seulement si la famille $\left(p \cdot X_{s}\right)_{s \in S}$ est eutactique (resp. semi-eutactique, faiblement eutactique) dans $F^{\Pi}$. Par exemple si $F^{\Pi}=\{0\}$, alors $\mathcal{F}$ est eutactique.

Preuve. - Il est clair que $p \cdot x=|\Pi|^{-1} \sum_{\pi \in \Pi} \pi \cdot x$ pour tout $x \in E$. Par suite, sachant que $\mathcal{F}$ est stable par $\Pi$, toute relation d'eutaxie (resp. de semieutaxie, d'eutaxie faible) entre les $\left(p \cdot X_{s}\right)_{s \in S}$ donne une relation analogue entre les $\left(X_{s}\right)_{s \in S}$. Inversement, les relations d'eutaxie se projettent toujours sur les sous-espaces.

Corollaire 1.3. - Soit $V$ une variété riemannienne munie d'un système de fonctions longueur $\left(f_{s}\right)$ stable (pour la composition) par l'action d'un groupe fini $\Pi$ d'isométries de $V$. Alors tout point fixe isolé $p$ de $\Pi$ est eutactique.

Preuve. - L'action linéaire tangente de $\Pi$ sur $T_{p} V$ (qui laisse stable les gradients $\nabla f_{s}(p)$ pour $\left.s \in S_{p}\right)$ est conjuguée à l'action de $\Pi$ au voisinage de $p$, donc ne peut fixer que l'origine de $T_{p} V$. 
1.2. Propriétés géométriques. Points non dégénérés et théorème de Voronoï. - Perfection et eutaxie ne dépendent que de la structure différentielle de $V$ et des dérivées premières des fonctions longueur. Pour exploiter des propriétés d'ordre plus élevé, il est souvent utile d'introduire sur $V$ des structures géométriques additionnelles. À partir d'ici nous supposerons donc que $V$ est munie d'une connexion $\nabla$ sur $T V$, par exemple la connexion de Levi-Civita d'une métrique riemannienne. Dans toute la suite, les connexions $\nabla$ seront supposées géodésiques (deux points quelconques de $V$ peuvent toujours être reliés par une géodésique de $\nabla)$, mais pas forcément complètes. Par exemple la métrique de Weil-Petersson sur l'espace de Teichmüller $\mathcal{T}_{g}(g>1)$ est géodésique non complète. Une fonction $f: V \rightarrow \mathbb{R}$ est (strictement) convexe si pour toute géodésique $\gamma$ de la connexion, $f \circ \gamma$ est (strictement) convexe. Nous introduisons maintenant une propriété plus générale et plus souple que la convexité.

DÉFinition 1.4. - Soit $I$ un intervalle de $\mathbb{R}$. Une fonction $f: I \rightarrow \mathbb{R}$ de classe $\mathcal{C}^{1}$ est convexö̈dale (resp. strictement convexö̈dale) si tout point critique de $f$ (i.e. zéro de $f^{\prime}$ ) est un minimum local (resp. un minimum local strict).

Soit $V$ une variété munie d'une connexion. Une fonction $f: V \rightarrow \mathbb{R}$ de classe $\mathcal{C}^{1}$ est (strictement) convexö̈dale si la fonction $f \circ \gamma$ est (strictement) convexoïdale pour toute géodésique $\gamma$.

Contrairement à la convexité, cette propriété est invariante par changement de paramétrage (composition à la source par un $\mathcal{C}^{1}$-difféomorphisme de $I$ ) et par composition au but par un $\mathcal{C}^{1}$-difféomorphisme croissant. Dans le cas d'une variété, on a donc une notion qui ne dépend que des images des géodésiques (« lieux géométriques ») et non de leur paramétrage (voir $\S 2.3$ pour un exemple naturel).

Soit $f: I \rightarrow \mathbb{R}$ de classe $\mathcal{C}^{1}$ convexoïdale et soit $[a, b] \subset I$. On a l'alternative suivante : ou bien $f$ est constante sur $[a, b]$, ou bien $f(t)<\max \{f(a), f(b)\}$ pour tout $t \in] a, b\left[\right.$. De là il résulte que l'ensemble $K_{f}$ des points critiques de $f$, s'il est non vide, est connexe et relativement fermé dans $I$, et réduit à un point dans le cas strict; de plus $f$ est constante sur $K_{f}$, et si on pose $f\left(K_{f}\right)=\{m\}$, on a $f>m$ sur $I \backslash K_{f}$. Si $f$ est analytique, elle est soit constante, soit strictement convexoïdale.

Remarque 1.3. - En remplaçant dans la définition 1.4 «point critique» par « extrémum local intérieur à $I$ » on pourrait se contenter de prendre $f$ continue. Les propriétés énoncées ci-dessus restent valables. Cependant, la définition 1.4 prend en compte tous les points critiques (dans le cas $\mathcal{C}^{1}$ ), ce qui est nécessaire pour la preuve de la proposition fondamentale 1.6. 
DÉfinition 1.5. - Soit $V$ une variété munie d'une connexion et d'une famille de fonctions longueur $\left(f_{s}\right)_{s \in C}$ convexoïdales. Un point $p$ de $V$ est dit non dégénéré si pour tout rayon géodésique $\gamma$ issu de $p$ ( $\gamma$ est définie sur un sousintervalle $I$ de $\left[0, \infty[\right.$ avec $0 \in I$ et $\gamma(0)=p)$, l'une des fonctions $f_{s} \circ \gamma\left(s \in S_{p}\right)$ est strictement convexoïdale au voisinage de 0 (c'est-à-dire non constante au voisinage de 0 ).

Cette propriété dépend évidemment de la connexion choisie. Si les $f_{s}$ sont strictement convexö̈dales, tous les points sont automatiquement non dégénérés (voir par exemple les $\S \S 2.3$ et 2.12). Quand les $f_{s}$ sont convexes, ce qui est le cas le plus courant, il suffit (avec les notations de la définition) que $\sum_{s \in S_{p}} f_{s} \circ \gamma$ soit strictement convexe.

EXemple 1.4 (réseaux et familles réseaux). - Soit $P_{n}$ l'espace des matrices de Gram de déterminant 1 (voir $\S 2.2$ ). Si $A \in P_{n}$ et $s \in \mathbb{Z}^{n}$, on pose $f_{s}(A)=$ $A[s]$. Pour la connexion introduite au $\S 2.3$ qui rend les $f_{s}$ strictement convexö̈dales, tous les points sont non dégénérés. Pour la structure riemannienne de $P_{n}$, on voit facilement qu'un point $A$ est non dégénéré si et seulement si le rang de l'ensemble $S_{A}$ de ses vecteurs minimaux est supérieur ou égal à $n-1$. Cette condition entraîne d'ailleurs (proposition 2.3) la non dégénérescence de $A$ relativement à toute sous-variété $V$ totalement géodésique de $P_{n}$, ou famille de réseaux; mais elle n'est pas nécessaire : il existe par exemple un réseau orthogonal non dégénéré avec $S_{A}$ de rang 1 (exemple 2.15). La traduction algébrique de la non dégénérescence relativement à $V$ est donnée à la proposition 2.3 (voir également le théorème $2, \S 2.8$ pour un critère concernant les réseaux autoduaux).

Proposition 1.4. - Soit $V$ munie de fonctions longueur convexoïdales. Alors tout point parfait est non dégénéré.

Preuve. - Si $p$ est un point dégénéré, il existe un germe de géodésique $\gamma$ issu de $p$ sur lequel l'invariant $\mu$ défini par (1.1) est constant. D'où l'existence d'un vecteur non nul orthogonal à toutes les $\left(d f_{s}\right)_{s \in S_{p}}$ et $p$ ne peut être parfait.

La notion de point non dégénéré nous sera utile pour préciser certains résultats de finitude concernant les points eutactiques. Elle est aussi liée à la caractérisation des maxima locaux de $\mu=\min _{s \in C} f_{s}$. Un maximum local (strict) de $\mu$ est appelé point (strictement) extrême. Rappelons (cf. [5]) que si les fonctions longueur sont convexes (convexoïdales suffit), un point est strictement extrême si et seulement s'il est parfait et eutactique. Nous dirons que le théorème de Voronoï est vérifié si extrême équivaut à parfait et eutactique. Dans ce cas les maxima locaux de $\mu$ sont tous stricts.

Proposition 1.5. - Soit $V$ munie de fonctions longueur convexö̈dales. Alors les propriétés suivantes sont équivalentes : 
a) le théorème de Voronoï est vérifié,

ß) tout point extrême est non dégénéré,

$\gamma)$ tout point extrême est parfait.

Le théorème de Voronoï est donc vérifié dès que les fonctions longueur sont strictement convexoïdales.

Preuve. - Il est clair que $\alpha$ ) implique $\gamma$ ), et (proposition 1.4) que $\gamma$ ) implique $\beta$ ). Soit $p$ extrême qui n'est pas à la fois parfait et eutactique. Alors il existe un vecteur $X \in T_{p} V$ non nul faiblement positif pour $\left(\mathrm{d} f_{s}(p)\right)_{s \in S_{p}}$ (lemme 1.1, assertion 1). Comme $p$ est extrême $X$ ne peut être positif, i.e. l'ensemble $T_{1}=\left\{s \in S_{p} ; \mathrm{d} f_{s}(p) \cdot X=0\right\}$ est non vide. Soit $\gamma$ la géodésique issue de $p$ de vitesse initiale $X$. Les $f_{s}$ étant convexoïdales et $p$ extrême, il existe un sous-ensemble non vide $T_{2}$ de $T_{1}$ tel que $f_{s} \circ \gamma(t)=\mu(p)\left(s \in T_{2}, t>0\right.$ petit $)$ et $S_{\gamma(t)}=T_{2}$; les points $\gamma(t)$ correspondants sont extrêmes et dégénérés.

Notre preuve s'inspire du théorème 4.5, (ii) de [10] et donne dans ce cas particulier des familles de réseaux un résultat plus précis (voir proposition 2.3) puisqu'une forme extrême peut être un point non dégénéré sans que ses vecteurs minimaux engendrent $\mathbb{R}^{n}$, ni même un hyperplan (un exemple est donné au $\S 2.2$ ). Cependant cette propriété algébrique plus forte que la non dégénérescence est satisfaite pour les réseaux extrêmes orthogonaux ou symplectiques $[10$, th. 7.6$]$.

Remarques 1.5. - 1) Le théorème de Voronoï ne dépend que de la structure différentielle de $V$. Quand il est satisfait, on voit donc (proposition 1.5) que les points extrêmes sont non dégénérés pour toute connexion sur $V$ qui rend les fonctions longueur convexoïdales. La caractérisation $\gamma$ ) a l'avantage de ne pas faire intervenir explicitement la structure géométrique, mais $\beta$ ) est plus faible et plus facilement vérifiable.

2) L'équivalence entre $\alpha$ ) et $\gamma$ ), de formulation purement différentielle, n'est plus vraie en dehors du cadre géométrique. Ainsi pour $V=\mathbb{R}, f_{1}(x)=\exp (x)$ et $f_{2}(x)=\left(\frac{1}{2} x^{2}+x+1\right) \exp (-x)$, l'unique point extrême $p=0$ est parfait mais pas eutactique.

3) Même dans le cadre géométrique, extrême et parfait n'entraîne pas eutactique (exemple: $V=\mathbb{R}, f_{1}(x)=x^{2}, f_{2}(x)=1$ et $p=1$ ).

1.3. Classes minimales et eutaxie. - Dans la théorie classique, on décompose l'espace des réseaux en « classes d'équivalence minimales » définies par les ensembles de vecteurs minimaux, décomposition très utile pour les questions de classification et de finitude (voir [28, IX]). Il est immédiat d'étendre cette idée au cadre général considéré ici. On associe à tout point $p$ de $V$ le sousensemble $S_{p}$ de $C$ (voir $\S 1.1$ ). On a ainsi une partition de $V: p$ et $q$ sont par définition dans la même classe si et seulement si $S_{p}=S_{q}$. Cette partition est 
localement finie (d'après les hypothèses faites sur les fonctions longueur) et ses classes forment un ensemble partiellement ordonné par l'inclusion de $C$.

Nous supposerons de plus, comme c'est le cas dans la plupart des situations naturelles, qu'il existe un groupe discret $\Gamma$ de difféomorphismes de $V$ préservant la connexion de $V$ (ou agissant isométriquement si $V$ est riemannienne) et agissant à droite sur $C$ de sorte que $f_{s} \circ \gamma=f_{s . \gamma}(s \in C, \gamma \in \Gamma)$. Alors la partition précédente de $V$ est $\Gamma$-invariante et induit une partition de $\Gamma \backslash V$ dont les classes forment également un ensemble partiellement ordonné. Nous adopterons la terminologie suivante : les classes de $V$ seront appelées classes minimales, celles de $\Gamma \backslash V$ classes d'équivalence minimales. Par exemple pour les réseaux, les vecteurs minimaux induisent une partition $\mathrm{SL}_{n}(\mathbb{Z})$-invariante de l'espace des formes $P_{n}$ et donc une partition de l'espace des réseaux $\mathrm{SL}_{n}(\mathbb{Z}) \backslash P_{n}$.

Soit $\mathcal{C}$ une classe minimale définie par un sous-ensemble $S$ de $C$. On pose

$$
\mathcal{C}^{\vee}=\left\{p \in V ; S_{p} \supset S\right\} .
$$

Il s'agit d'une partie fermée de $V$ qui est réunion de classes minimales et qui contient l'adhérence $\overline{\mathcal{C}}$ de $\mathcal{C}$. En général $\mathcal{C}^{\vee}$ ne coïncide pas avec $\overline{\mathcal{C}}$ (exemple : $V=\mathbb{R}, f_{1}(x)=\left(\frac{1}{2} x\right)^{2}, f_{2}(x)=(x-1)^{2}, f_{3}(x)=(x-3)^{2}$ et $\left.S=\{1\}\right)$.

Proposition 1.6. - Soit $\mathcal{C}$ une classe minimale et soit $p$ un point de $\mathcal{C}$.

1) On suppose que les $f_{s}$ sont strictement convexö̈dales (par exemple strictement convexes).

1a) Si $p$ est semi-eutactique, alors $p$ est un minimum strict de $\mu_{\mid \mathcal{C}^{v}}$.

1b) $S_{i} \mathcal{C}^{\vee}$ (resp. $\left.\mathcal{C}\right)$ est géodésique et $p$ faiblement eutactique, alors $p$ est un minimum strict de $\mu_{\mid \mathcal{C} \vee}\left(\right.$ resp. de $\left.\mu_{\mid \mathcal{C}}\right)$.

En particulier toute classe minimale (resp. toute classe minimale géodésique) contient au plus un point semi-eutactique (resp. faiblement eutactique).

2) On suppose que les $f_{s}$ sont convexö̈dales (par exemple convexes).

2a) Si p est semi-eutactique (resp. eutactique et non dégénéré), alors $p$ est un minimum (resp. un minimum strict) de $\mu_{\mid \mathcal{C}^{\vee}}$.

2b) Si $\mathcal{C}^{\vee}$ est géodésique et $p$ faiblement eutactique (resp. faiblement eutactique et non dégénéré), alors $p$ est un minimum (resp. un minimum strict) de $\mu_{\mid \mathcal{C}^{\vee}}$. On a le même énoncé avec $\mathcal{C}$ à la place de $\mathcal{C}^{\vee}$.

En particulier si une classe minimale (resp. une classe minimale géodésique) contient un point eutactique et non dégénéré (resp. faiblement eutactique et non dégénéré), c'est l'unique point semi-eutactique (resp. faiblement eutactique) de cette classe.

Preuve. - Notons $S$ le sous-ensemble de $C$ qui définit la classe $\mathcal{C}$. Dans toute la suite de la preuve, on pourrait remplacer $\mathcal{C}^{\vee}$ par $\mathcal{C}$. Soit $q \in \mathcal{C}^{\vee}$ distinct de $p$ avec $\mu(q) \leq \mu(p)$. Il existe une géodésique $\gamma$ telle que $\gamma(0)=q$ et $\gamma(1)=p$ (connexion géodésique). Comme $f_{s}$ est convexoïdale, on doit avoir ou bien $f_{s} \circ \gamma$ constante sur $[0,1]$, ou bien $\left(f_{s} \circ \gamma\right)^{\prime}(1)>0(s \in S)$. Si $\mathcal{C}^{\vee}$ est géodésique, on 
peut supposer que $\gamma$ est incluse dans $\mathcal{C}^{\vee}$ et toutes les $f_{s} \circ \gamma$ sont égales $(s \in S)$; posons dans ce cas $c=\left(f_{s} \circ \gamma\right)^{\prime}(1)$ indépendant de $s \in S$.

1a) On a cette fois $\left(f_{s} \circ \gamma\right)^{\prime}(1)>0$ pour tout $s \in S$ (cas strict), donc le vecteur $\gamma^{\prime}(1)$ est positif pour $\left(\mathrm{d} f_{s}(p)\right)_{s \in S}$ et $p$ ne peut être semi-eutactique (lemme 1.1,3).

1b) Cette assertion résulte de $2 \mathrm{~b})$ car tout point est non dégénéré.

2a) Si $p$ est non dégénéré, l'une des $f_{s} \circ \gamma(s \in S)$ est strictement convexö̈dale près de $p$, donc $\gamma^{\prime}(1)$ est semi-positif et $p$ ne peut être eutactique (lemme 1.1,2). Par ailleurs si $\mu(q)<\mu(p)$, alors $\gamma^{\prime}(1)$ est positif et $p$ est non semi-eutactique (lemme 1.1,3).

2b) Supposons $p$ faiblement eutactique. Si $\mu(q)<\mu(p)$ ou si $p$ est non dégénéré, on a $c>0$ en contradiction avec le lemme 1.1,4). D'où le résultat.

Note 1.6. - Les familles de réseaux étudiées dans [10] sont paramétrées dans $P_{n}$ par des orbites $V$ de sous-groupes de Lie connexes $G$ de $\mathrm{SL}_{n}(\mathbb{R})$ stables par transposition; associées à des images isométriques de $G G^{t}$ (voir [5, 2.4]), elles sont totalement géodésiques dans $P_{n}$ (voir [20, p.131]). Noter que les formes parfaites de $V$ et celles dont les vecteurs minimaux engendrent $\mathbb{R}^{n}$ sont des cas particuliers de points non dégénérés relativement à $V$ (proposition 1.4 et 2.3). Mais il existe aussi des familles naturelles avec $A \in V$ eutactique non dégénéré et $\operatorname{rang}\left(S_{A}\right)=1$ (voir exemple 2.15 pour les réseaux orthogonaux). En appliquant 2a) on retrouve donc le théorème 5.2 de [10]. On voit que ce résultat reflète des propriétés géométriques et n'est pas lié à la nature algébrique particulière des familles de réseaux.

Exemple 1.7. - La propriété d'avoir des classes minimales géodésiques paraît plutôt exceptionnelle. Cependant elle est vérifiée pour certains exemples intéressants : les П-réseaux avec la connexion introduite au $\S 2.3$, l'invariant d'Hermite isotrope des réseaux orthogonaux lorentziens (voir [4]), les familles hyperboliques de réseaux symplectiques (voir [6]). Dans ces trois cas les fonctions longueur sont strictement convexoïdales. De plus elles sont déterminées le long des géodésiques par leur jet d'ordre 1 en un point. On en déduit qu'un extrémum local $p$ de $\mu_{\mid \mathcal{C}}(\mathcal{C}$ classe minimale) est nécessairement faiblement eutactique : voir l'argument dans la preuve de la proposition 2.5, 3). Avec 1b), on a donc que $p$ est un minimum de $\mu_{\mid \mathcal{C}}$ si et seulement si $p$ est faiblement eutactique (pour les réseaux usuels, c'est le théorème 3.4 de [11]).

Corollaire 1.7. - Si les $f_{s}$ sont strictement convexö̈dales (resp. et si les classes minimales sont géodésiques), alors l'ensemble des points semi-eutactiques (resp. faiblement eutactiques) est discret.

Si les $f_{s}$ sont convexoïdales (resp. et si les classes minimales sont géodésiques), alors l'ensemble des points eutactiques et non dégénérés (resp. faiblement 
eutactiques et non dégénérés) est discret. En particulier l'ensemble des points parfaits et eutactiques est discret.

Preuve. - Les classes minimales forment une partition localement finie. Il suffit donc, dans chaque cas, d'appliquer les résultats d'unicité de la proposition 1.6.

1.4. Propriétés d'isolement. - Nous établissons ici que tous les points remarquables (parfaits, eutactiques, ...) sont des points isolés de certains sousensembles de $V$. Dans les applications il s'agira de sous-ensembles de nature algébrique, d'où des résultats de finitude et d'algébricité (voir lemme 1.11).

Pour tout sous-ensemble $S$ de $C$, notons $\widetilde{\mathcal{C}}_{S}$ l'ensemble des points $x$ de $V$ tels que $f_{s}(x)$ soit indépendant de $s \in S$. Quand $\underset{\widetilde{C}}{S}=S_{p}$, la classe minimale de $p$ est un sous-ensemble relativement ouvert de $\widetilde{\mathcal{C}}_{S}$.

Proposition 1.8. - Soient $p$ un point parfait et $S=S_{p}$. Alors $p$ est un point isolé de $\widetilde{\mathcal{C}}_{S}$ et de sa classe minimale $\mathcal{C}_{S}$. Si $\mathcal{C}_{S}$ est connexe, on a donc $\mathcal{C}_{S}=\{p\}$.

Preuve. - On munit $V$ d'une métrique riemannienne et on note $\exp _{p}$ l'exponentielle en $p\left(\exp _{p}(0)=p\right.$ et $\left.\mathrm{d}_{\exp }(0)=\mathrm{Id}\right)$. Soit $p_{n}=\exp _{p}\left(t_{n} X_{n}\right)$ une suite de points de $\widetilde{\mathcal{C}}_{S}$ avec $\left|X_{n}\right|=1 t_{n}>0$ qui tend vers $0(n \rightarrow \infty)$. On peut supposer de plus que $X_{n}$ converge vers $X \in T_{p} V,|X|=1$. Comme $f_{s}$ est de classe $\mathcal{C}^{1}$, on a

$$
f_{s} \circ \exp _{p}(h)=f_{s}(p)+\mathrm{d} f_{s}(p) \cdot h+|h| \epsilon_{s}(h) \quad \text { avec } \quad \lim _{h \rightarrow 0} \epsilon_{s}(h)=0 .
$$

Par suite

$$
\frac{1}{t_{n}}\left(f_{s}\left(p_{n}\right)-f_{s}(p)\right)=\mathrm{d} f_{s}(p) \cdot X_{n}+\epsilon_{s}\left(t_{n} X_{n}\right) .
$$

D'où l'on voit $(n \rightarrow \infty)$ que $\mathrm{d} f_{s}(p) \cdot X$ est indépendant de $s \in S$, en contradiction avec la perfection de $p$.

Remarques 1.8. - 1) Il existe des points isolés dans $\mathcal{C}_{S}$ non parfaits (exemple : $V=\mathbb{R}, f_{1}(x)=x^{2}, f_{2}(x)=x^{4}$ et $p=0$ ).

2) Il est possible que des points parfaits d'une classe $\mathcal{C}_{S}$ s'accumulent sur un point parfait d'une autre classe $\mathcal{C}_{T}$ ( $T$ contenant strictement $S$ ). On peut construire des exemples où $V$ est un intervalle de $\mathbb{R}$ avec trois fonctions longueur (que l'on peut choisir strictement convexes).

Nous appellerons rang (vectoriel) d'un point $p$ de $V$ par rapport à $S$, noté $\operatorname{rgv}^{S}(p)$, le rang vectoriel de la famille $\left(\mathrm{d} f_{s}(p)\right)_{s \in S}$ dans l'espace cotangent $T_{p}^{*}$, et rang de $p$ le rang par rapport à $S_{p}$. Pour $0 \leq r \leq \operatorname{dim} V$, on pose

$$
\mathcal{R}_{\leq r}^{S}=\left\{x \in V ; \operatorname{rgv}^{S}(x) \leq r\right\} .
$$

TOME $133-2005-\mathrm{N}^{\mathrm{O}} 2$ 
Proposition 1.9. - On suppose que les $f_{s}$ sont convexö̈dales (resp. et que les classes minimales sont géodésiques). Soit $p \in V$ de rang $r$ et soit $S=S_{p}$. Si $p$ est eutactique et non dégénéré (resp. faiblement eutactique et non dégénéré), alors il est isolé dans $\widetilde{\mathcal{C}}_{S} \cap \mathcal{R}_{\leq r}^{S}$ (si les $f_{s}$ sont strictement convexoïdales, on peut bien sûr omettre non dégénéré).

Lemme 1.10. - Soit E un $\mathbb{R}$-espace vectoriel de dimension finie et soit $S$ un ensemble fini. Posons :

$$
\begin{aligned}
A_{\leq r} & =\left\{\mathcal{F} \in E^{S} ; \operatorname{rang}(\mathcal{F}) \leq r\right\} \quad(0 \leq r \leq \operatorname{dim} E), \\
F E_{r} & =\left\{\mathcal{F} \in E^{S} ; \mathcal{F} \text { faiblement eutactique de } \operatorname{rang} r\right\}, \\
E_{r} & =\left\{\mathcal{F} \in E^{S} ; \mathcal{F} \text { eutactique de rang } r\right\} .
\end{aligned}
$$

Alors $F E_{r}$ et $E_{r}$ sont ouverts dans $A_{\leq r}$.

Preuve de la proposition. - Soit $\mathcal{C}_{S}$ la classe minimale de $p$ (ouverte dans $\widetilde{\mathcal{C}}_{S}$ ) et soit $\mathcal{E} u t_{r}$ (resp. $\mathcal{F E} u t_{r}$ ) l'ensemble des points eutactiques (resp. faiblement eutactiques) de rang $r$ de $V$. D'après le lemme, et comme les $f_{s}$ sont $\mathcal{C}^{1}$, les ensembles $\mathcal{C}_{S} \cap \mathcal{E} u t_{r}$ et $\mathcal{C}_{S} \cap \mathcal{F} \mathcal{E} u t_{r}$ sont ouverts dans $\widetilde{\mathcal{C}}_{S} \cap \mathcal{R}_{\leq r}^{S}$. Autrement dit, si $p$ est eutactique (resp. faiblement eutactique) tous les points de $\widetilde{\mathcal{C}}_{S} \cap \mathcal{R}_{\leq r}^{S}$ voisins de $p$ le sont aussi et sont dans $\mathcal{C}_{S}$. Pour conclure il suffit d'appliquer (dans chaque cas) les résultats d'unicité de la proposition 1.6.

Preuve du lemme. - On prendra $E$ eulidien et on identifiera $E^{S}$ avec $E^{N}$ où $N$ est le cardinal de $S$. Si $\mathcal{F}=\left(X_{1}, \ldots, X_{N}\right) \in A_{\leq r}$ est de rang $r$, on peut supposer que ses $r$ premiers vecteurs forment une base du sous-espace vectoriel engendré par $\mathcal{F}$. Il en est alors de même pour toute famille $\mathcal{G} \in A_{\leq r}$ voisine de $\mathcal{F}$. Soit $\left(\mathcal{F}^{n}\right)_{n \in \mathbb{N}}$ une suite de $A_{\leq r}$ qui converge vers $\mathcal{F}$ faiblement eutactique de rang $r$. Le rang affine de $\mathcal{F}$ vaut aussi $r$, de sorte que $N \geq r+1$.

Supposons que les $\mathcal{F}^{n}=\left(X_{1}^{n}, \ldots, X_{N}^{n}\right)$ ne sont pas faiblement eutactiques, i.e. il n'existe pas de relation $\sum_{1 \leq j \leq N} \alpha_{j}^{n} X_{j}^{n}=0$ avec $\sum_{1 \leq j \leq N} \alpha_{j}^{n} \neq 0$. On a donc, pour $j>r$,

$$
X_{j}^{n}=\sum_{1 \leq k \leq r} \lambda_{j, k}^{n} X_{k}^{n} \quad \text { avec } \quad \sum_{1 \leq k \leq r} \lambda_{j, k}^{n}=1 .
$$

On a aussi $X_{j}=\sum_{1 \leq k \leq r} \lambda_{j, k} X_{k}$ et nécessairement la suite $\left(\lambda_{j, k}^{n}\right)_{n \in \mathbb{N}}$ converge vers $\lambda_{j, k}$. Par suite

$$
\sum_{1 \leq k \leq r} \lambda_{j, k}=1 \quad(j>r)
$$

Cela étant, considérons une relation $\sum_{1 \leq j \leq N} \alpha_{j} X_{j}=0$, c'est-à-dire

$$
\sum_{1 \leq j \leq r} \alpha_{j} X_{j}+\alpha_{r+1}\left(\sum_{1 \leq k \leq r} \lambda_{r+1, k} X_{k}\right)+\cdots+\alpha_{N}\left(\sum_{1 \leq k \leq r} \lambda_{N, k} X_{k}\right)=0 .
$$

BULlETIN DE LA SOCIÉTÉ MATHÉMATIQUE DE FRANCE 
Alors on a $\alpha_{k}+\sum_{j>r} \alpha_{j} \lambda_{j, k}=0$ pour tout $k=1, \ldots, r$. D'où

$$
\sum_{k \leq r} \alpha_{k}+\sum_{k \leq r} \sum_{j>r} \alpha_{j} \lambda_{j, k}=\sum_{1 \leq j \leq N} \alpha_{j}=0
$$

ce qui est absurde puisque $\mathcal{F}$ est faiblement eutactique. On conclut que $F E_{r}$ est ouvert dans $A_{\leq r}$.

Supposons maintenant que les $\mathcal{F}^{n}$ ne sont pas eutactiques, i.e. (lemme 1.1) il existe un vecteur $X^{n}$ semi-positif pour $\mathcal{F}^{n}$ :

$$
\left\langle X_{j}^{n}, X^{n}\right\rangle \geq 0 \quad(1 \leq j \leq N) \quad \text { et } \sum_{1 \leq j \leq N}\left\langle X_{j}^{n}, X^{n}\right\rangle=1 .
$$

On peut choisir $X^{n}$ de la forme $X^{n}=\sum_{1 \leq k \leq r} \lambda_{k}^{n} X_{k}^{n}$ en projetant orthogonalement. Les $\left(\lambda_{k}^{n}\right)_{1 \leq k \leq r}$ sont déterminés par le système linéaire

$$
\sum_{1 \leq k \leq r} \lambda_{k}^{n}\left\langle X_{k}^{n}, X_{j}^{n}\right\rangle=\left\langle X^{n}, X_{j}^{n}\right\rangle \quad(1 \leq j \leq r),
$$

d'où l'on voit que les suites $\left(\lambda_{k}^{n}\right)_{n \in \mathbb{N}}$ sont bornées (le déterminant de Gram de $\left(X_{j}^{n}\right)_{1 \leq j \leq r}$ est minoré par un réel $>0$ et $\left(\left\langle X^{n}, X_{j}^{n}\right\rangle\right)_{n \in \mathbb{N}}$ est bornée par (1.3)). On peut donc supposer que les suites $\left(\lambda_{k}^{n}\right)_{n \in \mathbb{N}}$ convergent, d'où un vecteur semi-positif pour $\mathcal{F}$. En conclusion, $E_{r}$ est ouvert dans $A_{\leq r}$.

1.5. Finitude et algébricité. — Les propriétés d'isolement du $\S 1.4$ (propositions 1.8 et 1.9) serviront à établir la finitude et l'algébricité des points remarquables (parfaits, eutactiques, ...). Nous rappelons ici un résultat couramment utilisé pour ce type de question. On note $\overline{\mathbb{Q}}$ la clôture algébrique de $\mathbb{Q}$ dans $\mathbb{C}$.

Lemme 1.11. - Soit $X(\mathbb{R}) \subset \mathbb{R}^{N}$ un ensemble algébrique défini par des polynômes à coefficients dans $\overline{\mathbb{Q}} \cap \mathbb{R}$. Alors les points isolés de $X(\mathbb{R})$ forment un ensemble fini inclus dans $X(\overline{\mathbb{Q}} \cap \mathbb{R})$.

Preuve. - Je remercie Q. Liu pour la preuve qui suit (voir une autre approche dans $[7, \S 3]$ ). Chaque point isolé est une composante connexe de $X(\mathbb{R})$ (pour la topologie ordinaire), d'où la finitude (voir [13, th. 2.4.5]). Soit $K=\overline{\mathbb{Q}} \cap \mathbb{R}$, soit $x=\left(x_{1}, \ldots, x_{N}\right)$ un point isolé de $X(\mathbb{R})$ n'appartenant pas à $K^{N}$ et soit $L=K\left(x_{1}, \ldots, x_{N}\right)$. On peut supposer, quitte à permuter les coordonnées, que l'on a $L=K\left(x_{1}, \ldots, x_{p}\right)$ ou que $L=K\left(x_{1}, \ldots, x_{p}\right)[\theta](1 \leq p<N)$ avec $x_{1}, \ldots, x_{p}$ algébriquement indépendants sur $K$ et $\theta$ racine simple d'un polynôme à coefficients dans $K\left(x_{1}, \ldots, x_{p}\right)$ :

$$
\theta^{m}+f_{1}\left(x_{1}, \ldots, x_{p}\right) \theta^{m-1}+\cdots+f_{m}\left(x_{1}, \ldots, x_{p}\right)=0
$$

où $f_{j} \in K\left(X_{1}, \ldots, X_{p}\right)$ pour $j=1, \ldots, m$. Pour $\alpha=\left(\alpha_{1}, \ldots, \alpha_{p}\right)$ voisin de $\left(x_{1}, \ldots, x_{p}\right)$, le polynôme $t^{m}+f_{1}(\alpha) t^{m-1}+\cdots+f_{m}(\alpha)$ admet une racine réelle $\theta(\alpha)$ qui tend vers $\theta$ quand $\alpha$ tend vers $\left(x_{1}, \ldots, x_{p}\right)$ (utiliser le théorème 
des fonctions implicites). On peut donc définir un morphisme de $K$-algèbres $\varphi_{\alpha}: L \rightarrow \mathbb{R}$ en posant $\varphi_{\alpha}\left(x_{i}\right)=\alpha_{i}$ pour $i=1, \ldots, p$ et $\varphi_{\alpha}(\theta)=\theta(\alpha)$ si $L=K\left(x_{1}, \ldots, x_{p}\right)[\theta]$. Il est clair que le point $\left(\varphi_{\alpha}\left(x_{1}\right), \ldots, \varphi_{\alpha}\left(x_{N}\right)\right)$ appartient à $X(\mathbb{R})$ et tend vers $x$ quand $\alpha$ tend vers $\left(x_{1}, \ldots, x_{p}\right)$, ce qui est absurde.

Soit $P_{n}$ l'espace des matrices de Gram unimodulaires (voir $\S 2.2$ ) et soit $D$ un sous-ensemble non vide de $\mathbb{Z}^{n} \backslash\{0\}$. Pour $A \in P_{n}$ on pose

$$
\mu^{D}(A)=\min _{s \in D} A[s] .
$$

Corollaire 1.12. - Soit $V$ une sous-variété lisse de l'espace $P_{n}$ définie par des équations polynômiales à coefficients dans $\overline{\mathbb{Q}} \cap \mathbb{R}$. Alors :

1) les points parfaits pour $\mu^{D}$ (relativement à $V$ ) sont algébriques sur $\mathbb{Q}$ et en nombre fini dans chaque classe minimale,

2) si $V$ est de plus complète totalement géodésique (resp. et si les classes minimales sont géodésiques), tout point eutactique non dégénéré (resp. faiblement eutactique non dégénéré) pour $\mu^{D}$ est algébrique sur $\mathbb{Q}$.

Preuve. - Soit $A \in V$ et soit $S=S_{A}\left(S \subset D \subset \mathbb{Z}^{n}\right.$ ). Alors $\widetilde{\mathcal{C}}_{S}$ (pris dans $V$ ) est un ensemble algébrique de $\mathbb{R}^{n(n-1) / 2}$ défini par des équations à coefficients dans $\overline{\mathbb{Q}} \cap \mathbb{R}$.

1) Si $A$ est parfait, il est isolé dans $\widetilde{\mathcal{C}}_{S}$ (proposition 1.8), d'où le résultat (lemme 1.11).

2) Notons $K_{A}$ le sous-corps de $\mathbb{R}$ engendré par $\overline{\mathbb{Q}} \cap \mathbb{R}$ et les coefficients de $A$. L'espace tangent $T_{A} V$ et le produit scalaire $\langle., .\rangle_{A}(2.4)$ sont définis sur $K_{A}$, de même que la projection $\langle., .\rangle_{A}$-orthogonale $\pi_{A}$ de $\operatorname{Sym}_{n}(\mathbb{R})$ (voir 2.3) sur $T_{A} V$ (prendre une base orthogonale de $T_{A} V$ formée de matrices à coefficients dans $\left.K_{A}\right)$. Comme le gradient en $A$ de la fonction $f_{s \mid V}\left(s \in \mathbb{Z}^{n}\right)$ vaut $\pi_{A}\left(s s^{t}\right)$, on en déduit que les ensembles $\mathcal{R}_{\leq r}^{S}(V)$ (1.4) sont algébriques définis sur $\overline{\mathbb{Q}} \cap \mathbb{R}$, ce qui permet de conclure (lemme 1.11 et proposition 1.9).

Remarque 1.9. - Ce corollaire s'applique à toutes les familles naturelles et intéressantes de réseaux, notamment aux réseaux autoduaux (symplectiques,

orthogonaux, ...), voir corollaire $2.12,2$ ). On prend généralement $D$ invariant par un sous-groupe de $\mathrm{SL}_{n}(\mathbb{Z})$ agissant sur $V$.

\section{Résultats de finitude}

2.1. Compacité dans l'espace des réseaux. - On rappelle que $P_{n}$ désigne l'espace des matrice de Gram $n \times n$ de déterminant 1 et que $\mathrm{SL}_{n}(\mathbb{R})$ opère sur $P_{n}$ par

$$
P \cdot A=P A P^{t} \quad\left(P \in \mathrm{SL}_{n}(\mathbb{R}), A \in P_{n}\right) .
$$

BULlETIN DE LA SOCIÉTÉ MATHÉMATIQUE DE FRANCE 
Le critère de compacité de Mahler affirme que l'invariant d'Hermite défini sur l'espace des réseaux (considérés à similitude directe près) $\mathrm{SL}_{n}(\mathbb{Z}) \backslash P_{n}$ est propre comme fonction à valeurs dans $] 0, \infty[$. Nous nous intéressons ici au critère de Mahler relatif pour des sous-variétés $V$ de $P_{n}$. Dans de nombreux cas intéressants, $V$ est une orbite d'un groupe algébrique $G(\mathbb{R}) \subset \mathrm{SL}_{n}(\mathbb{R})$. En utilisant les techniques usuelles d'étude des groupes arithmétiques, on établit un critère de compacité relatif.

DÉFINITION 2.1. - Soit $\varphi: \mathrm{SL}_{n}(\mathbb{C}) \rightarrow \mathrm{GL}_{N}(\mathbb{C})$ une représentation continue et soit $v$ un vecteur de $\mathbb{R}^{N}$. On suppose qu'il existe un réseau $L$ de $\mathbb{R}^{N}$ contenant $v$ et stable par $\varphi\left(\mathrm{SL}_{n}(\mathbb{Z})\right)$. Alors le groupe

$$
G(\mathbb{C})=\left\{P \in \mathrm{SL}_{n}(\mathbb{C}) ; \varphi(P) \cdot v=v\right\}
$$

sera appelé pseudo-algébrique, de même que $G(\mathbb{R})=G(\mathbb{C}) \cap \mathrm{SL}_{n}(\mathbb{R})$.

Par exemple les $\mathbb{Q}$-groupes algébriques réductifs ou ne possédant pas de caractère défini sur $\mathbb{Q}$ non trivial satisfont cette définition avec $\varphi$ algébrique sur $\mathbb{Q}$ et $L$ inclus dans $\mathbb{Q}^{N}$.

Proposition 2.1. - Soit $G(\mathbb{R})$ un groupe pseudo-algébrique. On pose

$$
G(\mathbb{Z})=G(\mathbb{R}) \cap \mathrm{SL}_{n}(\mathbb{Z}) .
$$

Alors, pour tout $A \in P_{n}$, l'application

$$
G(\mathbb{Z}) \backslash G(\mathbb{R}) \cdot A \longrightarrow \mathrm{SL}_{n}(\mathbb{Z}) \backslash P_{n}
$$

est propre et à fibres finies. En particulier on a un critère de Mahler relatif pour $V=G(\mathbb{R}) \cdot A$.

Remarque 2.1. - Si $A=P P^{t} \in P_{n}$ est la matrice de Gram du réseau $\Lambda=P^{t} \mathbb{Z}^{n}$ et $G$ un sous-groupe de $\mathrm{SL}_{n}(\mathbb{R})$, l'orbite $G \cdot A$ dans $P_{n}$ paramètre l'orbite $\left(P^{-1} G P\right)^{t}(\Lambda)=(G P)^{t} \mathbb{Z}^{n}$ dans l'espace des réseaux.

Preuve de la proposition. - Tout d'abord, l'application

$$
G(\mathbb{Z}) \backslash G(\mathbb{R}) \longrightarrow \mathrm{SL}_{n}(\mathbb{Z}) \backslash \mathrm{SL}_{n}(\mathbb{R})
$$

est propre. Cet énoncé est classique pour certains $\mathbb{Q}$-groupes algébriques (par exemple réductifs) qui sont pseudo-algébriques au sens de la définition 2.1. Mais c'est uniquement la description (2.2) (avec l'existence d'un réseau $L$ ) qui est utile et qui sera facilement et directement vérifiable pour les cas qui nous intéressent. Rappelons brièvement les arguments; on pourra par exemple consulter la preuve de la proposition 10.15 dans [31]. Posons provisoirement $\Gamma=\mathrm{SL}_{n}(\mathbb{Z})$. L'application (2.3) est la composée de la bijection continue

$$
h: G(\mathbb{Z}) \backslash G(\mathbb{R}) \longrightarrow \Gamma \backslash \Gamma G(\mathbb{R})
$$

suivie du plongement topologique

$$
j: \Gamma \backslash \Gamma G(\mathbb{R}) \longrightarrow \Gamma \backslash \mathrm{SL}_{n}(\mathbb{R})
$$

TOME $133-2005-\mathrm{N}^{\mathrm{O}} 2$ 
$(\Gamma G(\mathbb{R})$ est saturé). De l'existence de $L$, on déduit d'abord que $\varphi(\Gamma) \cdot v$ est fermé dans $\mathbb{R}^{N}$, donc $\Gamma G(\mathbb{R})=\left\{P \in \mathrm{SL}_{n}(\mathbb{R}) ; \varphi(P) \cdot v \in \varphi(\Gamma) \cdot v\right\}$ est fermé dans $\mathrm{SL}_{n}(\mathbb{R})$ et l'image de $j$ est fermée. Toujours avec l'existence de $L$, on voit que l'injection $G(\mathbb{R}) \rightarrow \Gamma G(\mathbb{R})$ est ouverte et par suite $h$ est un homéomorphisme. Finalement (2.3) est un plongement topologique d'image fermée.

Il reste à passer aux orbites des groupes dans $P_{n}$. Pour tout $A \in P_{n}$ on a un diagramme commutatif d'applications continues :

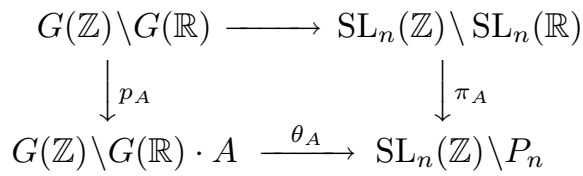

où $\pi_{A}\left(\mathrm{SL}_{n}(\mathbb{Z}) P\right)=\mathrm{SL}_{n}(\mathbb{Z}) \cdot(P \cdot A)$. Sachant que $p_{A}$ est surjective et que $(2.3)$ est propre, la propreté de $\theta_{A}$ résultera de celle de $\pi_{A}$. Or la propreté de $\pi_{A}$ équivaut à celle de $\pi_{I}$ que l'on établit sans problème. Enfin, les fibres de $\theta_{A}$ sont discrètes et compactes, donc finies.

ExEMPle 2.2 (familles hyperboliques de réseaux symplectiques, [6])

Soit $g \in \mathbb{N}^{*}$ et soit $M$ une matrice symétrique $g \times g$ définie positive. On considère dans $P_{2 g}$ la famille $\mathcal{F}_{M}$ des matrices $\left(\begin{array}{cc}a M & b I \\ b I & d M^{-1}\end{array}\right)$ avec $a>0, d>0$ et $a d-b^{2}=1$. Il s'agit d'une sous-variété totalement géodésique isométrique à un facteur près au demi-plan de Poincaré. Il est facile de voir que le groupe $G$ formé des matrices $\left(\begin{array}{cc}\alpha I & \beta M \\ \gamma M^{-1} & \delta I\end{array}\right)$ avec $\alpha \delta-\beta \gamma=1$ (isomorphe à $\mathrm{SL}_{2}(\mathbb{R})$ ) agit transitivement sur $\mathcal{F}_{M}$. Montrons que $G$ est de la forme $(2.2)$. Soit $\left(E_{j}\right)_{1 \leq j \leq g^{2}}$ une base du groupe abélien $M_{g}(\mathbb{Z})$ (donc du $\mathbb{C}$-espace vectoriel $M_{g}(\mathbb{C})$ ). Posons

$$
\varphi(P) \cdot\left(X_{0}, X_{1}, \ldots, X_{g^{2}}\right)=\left(P X_{0} P^{t}, P X_{1} P^{-1}, \ldots, P X_{g^{2}} P^{-1}\right)
$$

pour $P \in \mathrm{SL}_{2 g}(\mathbb{C})$ et $\left(X_{j}\right)_{0 \leq j \leq g^{2}} \in M_{2 g}(\mathbb{C})^{1+g^{2}}$, et

$$
v=\left(\left(\begin{array}{cc}
0 & I \\
-I & 0
\end{array}\right),\left(\begin{array}{cc}
M E_{1} & 0 \\
0 & E_{1} M
\end{array}\right), \ldots,\left(\begin{array}{cc}
M E_{g^{2}} & 0 \\
0 & E_{g^{2}} M
\end{array}\right)\right) .
$$

Alors on a $G(\mathbb{R})=G$ pour ces choix (poser $P=\left(\begin{array}{ll}A & B \\ C & D\end{array}\right) \cdots$ ). Si $M$ est entière, le réseau $L=M_{2 g}(\mathbb{Z})^{1+g^{2}}$ vérifie les hypothèses et on a un critère de compacité pour $G(\mathbb{Z}) \backslash \mathcal{F}_{M}$. Noter que dans ce cas $G(\mathbb{Z})$ est isomorphe à un sous-groupe de congruence de $\mathrm{SL}_{2}(\mathbb{Z})$ de type $\Gamma_{0}(m)$.

Corollaire 2.2. - Soit $G(\mathbb{R})$ pseudo-algébrique et soit $V=G(\mathbb{R}) \cdot A$ $\left(A \in P_{n}\right)$. On note $\mu$ l'invariant d'Hermite. Alors pour tout $\epsilon>0$, il n'existe qu'un nombre fini de classes d'équivalence minimales dans $G(\mathbb{Z}) \backslash V$ qui rencontrent l'ensemble $(G(\mathbb{Z}) \backslash V)_{\geq \epsilon}=\{\Lambda \in G(\mathbb{Z}) \backslash V ; \mu(\Lambda) \geq \epsilon\}$.

Preuve. - La partition de $G(\mathbb{Z}) \backslash V$ est localement finie et d'après la proposition 2.1 l'ensemble $(G(\mathbb{Z}) \backslash V)_{\geq \epsilon}$ est compact. 
2.2. Non dégénérescence dans les familles de réseaux. - Soit $P_{n}$ l'espace des matrices réelles symétriques positives $n \times n$ de déterminant 1 (matrices de Gram). Nous considérons ici $P_{n}$ comme espace symétrique riemannien, c'està-dire muni de la métrique

$$
\langle X, Y\rangle_{A}=\operatorname{Tr}\left(A^{-1} X A^{-1} Y\right) \quad\left(A \in P_{n}, X, Y \in T_{A} P_{n}\right)
$$

invariante par l'action $(2.1)$ de $\mathrm{SL}_{n}(\mathbb{R})$. On rappelle également que la géodésique issue de $A$ et de vitesse initiale $X$ est donnée par $\gamma_{X}(t)=A \exp \left(t A^{-1} X\right)$. La symétrie centrée en $A$ s'exprime par $\sigma_{A}(B)=A B^{-1} A\left(B \in P_{n}\right)$ et $\sigma_{I}$ correspond à la dualité des réseaux ( $I$ désigne la matrice identité).

Les fonctions longueur s'écrivent

$$
f_{s}(A)=A[s]=s^{t} A s \quad\left(A \in P_{n}, s \in \mathbb{Z}^{n}\right) .
$$

La finitude locale des $f_{s}$ 《petites » est immédiate à partir de l'inégalité $\left(u^{t} s\right)^{2} \leq A^{-1}[u] A[s]$ pour $u, s \in \mathbb{R}^{n}$ (voir aussi remarque 1.1). Un petit calcul permet d'expliciter le hessien $H f_{s}$ de $f_{s}$ :

$$
H f_{s}(A) \cdot(X, X)=A^{-1}[X s] \quad\left(A \in P_{n}, X \in T_{A} P_{n}\right) .
$$

Les $f_{s}$ sont donc convexes. Plus précisément, si $\gamma$ est une géodésique de $P_{n}$, alors $f_{s} \circ \gamma$ est soit strictement convexe, soit constante. En effet si $H f_{s}(A) \cdot(X, X)=0$ pour $X \neq 0$, on doit avoir $X s=0$ et $f_{s} \circ \gamma_{X}$ est constante d'après l'expression des géodésiques.

Proposition 2.3. - Soit $V$ une sous-variété totalement géodésique de $P_{n}$ et soit $A \in V$. Les propriétés suivantes sont équivalentes :

(i) Le point $A$ est non dégénéré relativement à $V$.

(ii) $\sum_{s \in S_{A}} f_{s}$ est radialement strictement convexe en $A$ sur $V$.

(iii) Si le noyau de $X \in T_{A} V$ contient $S_{A}$, alors $X=0$.

En particulier, si le rang des vecteurs minimaux de $A$ est supérieur ou égal à $n-1$, alors $A$ est non dégénéré relativement à $V$.

Preuve. - Le point $A \in V$ est dégénéré si et seulement s'il existe un rayon géodésique $\gamma$ de $V$ issu de $A$ tel que les $f_{s} \circ \gamma\left(s \in S_{A}\right)$ sont toutes constantes au voisinage de zéro. D'après ce qui précède, cela revient à dire que $\sum_{s \in S_{A}} f_{s} \circ \gamma$ n'est pas strictement convexe, ou encore qu'il existe une direction $X$ tangente à $V$ en $A$ dont le noyau contient $S_{A}$. Bien sûr $X(\neq 0)$ ne peut exister si $\operatorname{rang}\left(S_{A}\right) \geq n-1$ puisque $\operatorname{Tr}\left(A^{-1} X\right)=0$.

On se convainc facilement que la condition $\operatorname{rang}\left(S_{A}\right) \geq n-1$ n'est pas nécessaire pour la non dégénérescence dans $V$ (voir exemple 2.15). Voici un exemple avec $A$ extrême dans $V$ (et $\operatorname{dim} V>0$ !) : on prend $V=\mathcal{F}_{M}(\S 2.1)$ pour $M=[2,0,-1 ; 0,2,0 ;-1,0,2]$ et $A=3^{-1 / 2}\left(\begin{array}{ll}M & I \\ I & 4 M^{-1}\end{array}\right)\left(n=6\right.$ et $\left.\operatorname{rang}\left(S_{A}\right)=4\right)$. 
2.3. Une connexion naturelle sur l'espace des formes. - Nous introduisons ici une connexion sur $P_{n}$ qui constituera un outil techniquement bien adapté à l'étude des ПI-réseaux $(\S 2.4)$. On note $\operatorname{Sym}_{n}(\mathbb{R})\left(\operatorname{resp} . \operatorname{Sym}_{n}^{+}(\mathbb{R})\right)$ l'ensemble des matrices symétriques (resp. symétriques définies positives) de $M_{n}(\mathbb{R})$ et

$$
T P_{n}=\left\{(A, X) \in P_{n} \times \operatorname{Sym}_{n}(\mathbb{R}) ; \operatorname{Tr}\left(A^{-1} X\right)=0\right\}
$$

le fibré tangent de $P_{n}$. Fixons une forme linéaire $\ell \operatorname{sur}^{\operatorname{Sym}_{n}}(\mathbb{R})$ qui prend des valeurs strictement positives sur le cône $\operatorname{Sym}_{n}^{+}(\mathbb{R})$ (par exemple la trace Tr) et considérons la connexion $\nabla$ définie par le champ géodésique suivant sur $T P_{n}$ (on écrit uniquement la composante dans la fibre $T_{(A, X)} T P_{n}$ ) :

$$
\mathcal{Z}(A, X)=\left(X, \frac{1}{n} \operatorname{Tr}\left[\left(A^{-1} X\right)^{2}\right] A+2 \frac{\ell(X)}{\ell(A)} X\right) \quad(A, X) \in T P_{n} .
$$

Il est immédiat de vérifier que $\mathcal{Z}$ est bien un champ de vecteurs tangents à $T P_{n}$. Les géodésiques de $\nabla$ sont les solutions de l'équation différentielle

$$
A^{\prime \prime}=\frac{1}{n} \operatorname{Tr}\left[\left(A^{-1} A^{\prime}\right)^{2}\right] A+2 \frac{\ell\left(A^{\prime}\right)}{\ell(A)} A^{\prime} .
$$

La connexion $\nabla$ est naturelle car elle provient d'une carte affine globale de $P_{n}$. En effet, soit $\mathcal{D}=\left\{B \in \operatorname{Sym}_{n}^{+}(\mathbb{R}) ; \ell(B)=1\right\}$ et soit $\Phi$ le difféomorphisme de $P_{n}$ sur $\mathcal{D}$ défini par $\Phi(A)=A / \ell(A)$. Un petit calcul montre que $A$ est solution de $(2.6)$ si et seulement si $(A / \ell(A))^{\prime \prime}=0$, d'où l'on voit que les géodésiques de $\nabla$ correspondent via $\Phi$ aux segments affinement paramétrés de $\mathcal{D}$. La connexion $\nabla$ est donc uniquement géodésique (D est convexe) et a priori non complète (par exemple, $\mathcal{D}$ est borné si $\ell=\mathrm{Tr}$ ). Le comportement des fonctions longueur de $P_{n}$ sur les géodésiques de $\nabla$ est le suivant.

Lemme 2.4. - 1) Les fonctions longueur de $P_{n}$ sont strictement convexö̈dales pour $\nabla$.

2) Soient $f$ et $g$ les restrictions de deux fonctions longueur le long d'une géodésique de $\nabla$. Si $f$ et $g$ coïncident en deux points distincts, ou si $f$ et $g$ ont le même jet d'ordre 1 en un point, alors $f=g$.

Preuve. - Soit $A=A(t)$ une géodésique fixée de $\nabla$ et soit $\lambda=\frac{1}{n} \operatorname{Tr}\left[\left(A^{-1} A^{\prime}\right)^{2}\right]$. Les fonctions longueur $f_{s} \circ A(t)=A(t)[s]\left(s \in \mathbb{Z}^{n}\right)$ sont toutes solutions de la même équation différentielle

$$
f^{\prime \prime}=\lambda f+2 \frac{(\ell \circ A)^{\prime}}{\ell \circ A} f^{\prime}
$$

Si $t_{0}$ est un point critique de $f=f_{s} \circ A$, alors $f^{\prime \prime}\left(t_{0}\right)=\lambda\left(t_{0}\right) f\left(t_{0}\right)$ est strictement positif, d'où l'assertion 1$)$. Par ailleurs, deux solutions quelconques $f$ et $g$ de (2.7) sont liées par la relation

$$
\left(\frac{f^{\prime} g-g^{\prime} f}{(\ell \circ A)^{2}}\right)^{\prime}=0
$$

BULlETIN DE LA SOCIÉTÉ MATHÉMATIQUE DE FRANCE 
d'où $f^{\prime} g-g^{\prime} f=C_{f, g}(\ell \circ A)^{2}\left(C_{f, g} \in \mathbb{R}\right)$. Si $g>0$, la fonction $f / g$ est donc strictement croissante, strictement décroissante ou constante suivant que $C_{f, g}>0$, $C_{f, g}<0$ ou $C_{f, g}=0$, ce qui prouve l'assertion 2).

Noter que $\ell \circ A$ est solution de $\varphi^{\prime \prime}=\lambda \varphi+2 \varphi^{\prime 2} / \varphi$, c'est-à-dire

$$
\varphi\left(-\frac{1}{\varphi}\right)^{\prime \prime}=\lambda>0
$$

La fonction $-1 / \ell$ est donc strictement convexe pour $\nabla$, en particulier la forme linéaire $\ell\left(\right.$ sur $\left.P_{n}\right)$ est strictement log-convexe pour $\nabla$. Remarquer aussi que pour toute solution $f$ de (2.7) (notamment pour toute fonction longueur), on a $(f / \ell \circ A)^{\prime \prime}=0$ et $f / \ell \circ A$ est une fonction affine.

2.4. Réseaux avec action d'un groupe fini. - Soit $\rho: \Pi \rightarrow \mathrm{GL}_{n}(\mathbb{Z})$ une représentation intégrale d'un groupe fini $\Pi$. On pose $\rho^{\vee}=\left(\rho^{t}\right)^{-1}$ et on note $\mathbb{Z}_{\rho^{\vee}}^{n}$ le $\mathbb{Z}[\Pi]$-module associé. Considérons l'espace $P_{n}^{\rho}$ des formes $\rho$-invariantes :

$$
P_{n}^{\rho}=\left\{A \in P_{n} ; \rho(\pi) A \rho(\pi)^{t}=A, \forall \pi \in \Pi\right\} .
$$

Si $A=P P^{t} \in P_{n}^{\rho}$, la représentation $P^{-1} \rho P$ (à valeurs dans $\mathbb{O}_{n}(\mathbb{R})$ ) induit sur le réseau $\Lambda=P^{t} \mathbb{Z}^{n}$ une structure de $\mathbb{Z}[\Pi]$-module isomorphe à $\mathbb{Z}_{\rho^{\vee}}^{n}$ par $\left(P^{t}\right)^{-1}$. Les formes $\rho$-invariantes correspondent donc aux réseaux $\Lambda$ (de déterminant 1 , à isométrie directe près) munis d'une action isométrique de $\Pi$ (« П-réseaux ») et d'un isomorphisme de $\mathbb{Z}[\Pi]$-modules de $\mathbb{Z}_{\rho^{\vee}}^{n}$ sur $\Lambda$.

Exemple 2.3. - Les réseaux hermitiens complexes dans $\mathbb{C}^{m}$ ou hermitiens quaternioniens dans $\mathbb{H}^{m}$ pour $n=2 m, n=4 m$, voir remarque 2.8 .

Les propriétés générales des familles de formes munies de l'action d'un groupe fini sont rassemblées au $\S 2.5$. On voit en particulier (proposition 2.7) que $P_{n}^{\rho}$ est une sous-variété connexe non vide et totalement géodésique de l'espace symétrique $P_{n}$, que c'est une orbite du commutant $\mathrm{SL}_{n}^{\rho}(\mathbb{R})$ de $\rho$ dans $\mathrm{SL}_{n}(\mathbb{R})$, et que l'on a un critère de Mahler relatif pour $\mathrm{SL}_{n}^{\rho}(\mathbb{Z}) \backslash P_{n}^{\rho}$. Noter que d'après la preuve de la proposition 2.7, 3), $\mathrm{SL}_{n}^{\rho}$ est un groupe algébrique vérifiant (2.2).

Rappelons que les vecteurs minimaux d'une forme faiblement eutactique dans $P_{n}^{\rho}$ engendrent $\mathbb{R}^{n}$. En fait, l'eutaxie faible dans $P_{n}^{\rho}$ équivaut à l'eutaxie faible dans $P_{n}$ (voir [28, XI, prop. 3.3] ou plus généralement lemme 2.8) et de même pour la semi-eutaxie ou l'eutaxie.

Bien que $P_{n}^{\rho}$ possède de bonnes propriétés vis-à-vis de la connexion riemannienne de $P_{n}$, il est plus utile ici de munir $P_{n}$ de la connexion $\nabla$ définie au $\S 2.3$. Il est clair que $T P_{n}^{\rho}$ est stable par flot de $\mathcal{Z}$ (eq. (2.5)), autrement dit $P_{n}^{\rho}$ est totalement géodésique pour $\nabla$. Les fonctions longueur $f_{s \mid P_{n}^{\rho}}$ sont donc strictement convexö̈dales et on retrouve avec la proposition 1.5 le théorème de Voronoï pour $P_{n}^{\rho}$ (voir $[9$, th. 2.10]). 
Proposition 2.5. - Soit $\mathcal{C}$ une classe minimale de $P_{n}^{\rho}$ et soit $A \in \mathcal{C}$.

1) $\mathcal{C}$ et $\mathcal{C}^{\vee}$ (voir (1.2)) sont géodésiques pour $\nabla$.

2) $\mathcal{C}^{\vee}$ est l'adhérence de $\mathcal{C}$.

3) A est un extrémum local de $\mu_{\mid \mathcal{C}}$ si et seulement si $A$ est faiblement eutactique, si et seulement si $A$ est un minimum strict de $\mu_{\mid \mathcal{C} \vee}$.

4) $A$ est un maximum local de $\mu_{\mid \mathcal{C}}$ si et seulement si $A$ est parfait, si et seulement si $\mathcal{C}=\mathcal{C}^{\vee}=\{A\}$.

5) Soient $M=\sup \mu_{\mid \mathcal{C}}$ et $m=\inf \mu_{\mid \mathcal{C}}$. Alors $M$ est atteint dans $\mathcal{C}^{\vee}$, de même que $m$ si $m \neq 0$. On a $M \geq 1$ et $m=0$ ou $m \geq 1$.

6) $\mathcal{C}$ contient dans son adhérence un point parfait.

Preuve. - Notons $S$ le sous-ensemble de $\mathbb{Z}^{n}$ qui définit $\mathcal{C}$ et $\mathcal{C}^{\vee}$.

1) Soient $B$ et $C$ deux points de $P_{n}^{\rho}$ et soit $\gamma:[0,1] \rightarrow P_{n}^{\rho}$ la géodésique de $\nabla$ qui joint $B$ et $C$. On suppose que $S_{B} \cap S_{C} \neq \varnothing$. Alors pour $\left.t \in\right] 0,1[$ on a

$$
S_{\gamma(t)}=S_{B} \cap S_{C}
$$

En effet d'après le lemme $2.4,2)$ les fonctions $f_{s}\left(s \in S_{B} \cap S_{C}\right)$ coïncident et sont minimales sur $\gamma([0,1])$; de plus si $f_{s}$ est minimale en $\gamma(t)(0<t<1)$, alors $s \in S_{B} \cap S_{C}$ (considérer le 1 -jet en un point). Il résulte de $(2.8)$ que $\mathcal{C}$ et $\mathcal{C}^{\vee}$ sont géodésiques.

2) Si $B \in \mathcal{C}^{\vee}$ on choisit $C \in \mathcal{C}$ et d'après (2.8) on a $S_{\gamma(t)}=S_{C}=S$ pour tout $t \in] 0,1]$. Par suite $B$ appartient à l'adhérence de $\mathcal{C}$.

3) Soit $A$ un extrémum local de $\mu_{\mid \mathcal{C}}$ qui n'est pas faiblement eutactique. Par le lemme 1.1,4), il existe un vecteur tangent $X \in T_{A} P_{n}^{\rho}$ tel que $\mathrm{d} f_{s}(X)=c$, avec $c$ indépendant de $s \in S$ et non nul. Soit $\gamma:\left[0, \epsilon\left[\rightarrow P_{n}^{\rho}\right.\right.$ un germe de géodésique tel que $\gamma(0)=A$ et $\gamma^{\prime}(0)=X$. Les fonctions $f_{s} \circ \gamma(s \in S)$ coïncident (même 1-jet en $t=0$ ) et comme $S_{B}$ est inclus dans $S_{A}$ pour $B$ voisin de $A$, on a $\gamma([0, \epsilon]) \subset \mathcal{C}$. D'où une variation de $A$ dans $\mathcal{C}$ qui diminue (ou augmente) strictement $\mu$, ce qui est absurde.

Comme $\mathcal{C}^{\vee}$ est géodésique, tout point faiblement eutactique de $\mathcal{C}$ est un minimum strict de $\mu_{\mid \mathcal{C}^{\vee}}$ (proposition 1.6, 1b). Évidemment, un tel minimum est un extrémum local de $\mu_{\mid \mathcal{C}}$.

4) Soit $A$ un maximum local de $\mu_{\mid \mathcal{C}}$, donc faiblement eutactique par 3). Si $A$ n'est pas parfait, il existe $X \in T_{A} P_{n}^{\rho}(X \neq 0)$ tel que $\mathrm{d} f_{s}(X)=0(s \in S)$. D'où (voir la preuve de 3 ) une variation géodésique $\gamma$ de $A$ dans $\mathcal{C}$. Mais comme les fonctions longueur sont strictement convexoïdales, cette variation augmente strictement $\mu$, ce qui est absurde.

Si $A$ est parfait, alors $\mathcal{C}=\{A\}$ (voir 1 ) et prop. 1.8), et d'après 2 ) on a aussi $\mathcal{C}^{\vee}=\mathcal{C}=\{A\}$. Cette dernière relation entraîne que $A$ est un maximum local de $\mu_{\mid \mathcal{C}}$.

5) Notons $\pi: P_{n}^{\rho} \rightarrow \mathrm{SL}_{n}^{\rho}(\mathbb{Z}) \backslash P_{n}^{\rho}$ la projection naturelle ( $\pi$ est ouverte) et posons $\mu=\bar{\mu} \circ \pi$. Par compacité il existe un point $x$ dans l'adhérence de $\pi(\mathcal{C})$ 
tel que $\bar{\mu}(x)=M$. Soit $B \in \pi^{-1}(x)$ et soit $\mathcal{U}$ un voisinage ouvert de $B$ tel que $S_{C} \subset S_{B}$ pour tout $C \in \mathcal{U}$. Comme $\pi(\mathcal{U})$ rencontre $\pi(\mathcal{C})$, il existe $P \in \mathrm{SL}_{n}^{\rho}(\mathbb{Z})$ tel que $P \cdot \mathcal{U}$ rencontre $\mathcal{C}$. Soit $C=P \cdot B$ : alors clairement $S_{C}$ contient $S$ et $\mu(C)=M$. D'après 2) et 4), $C$ est parfait, donc $S_{C}$ engendre $\mathbb{R}^{n}$ et $M \geq 1$ par l'inégalité de Hadamard. Si $m>0$, on montre de même que $m$ est atteint dans $\mathcal{C}^{\vee}$ et que $m \geq 1$.

6) Cela résulte des assertions 2), 4) et 5).

Corollaire 2.6. - Modulo l'action de $\mathrm{SL}_{n}^{\rho}(\mathbb{Z})$ :

1) le nombre de classes minimales de $P_{n}^{\rho}$ est fini;

2) les points faiblement eutactiques de $P_{n}^{\rho}$ sont en nombre fini.

Preuve. - 1) La partition en classes est localement finie et d'après 5) toute classe d'équivalence minimale de $\mathrm{SL}_{n}^{\rho}(\mathbb{Z}) \backslash P_{n}^{\rho}$ rencontre le compact défini par $\mu \geq 1-\epsilon(0<\epsilon<1)$, d'où la finitude.

2) Comme les classes minimales sont géodésiques, le résultat se déduit de l'assertion 1) et de la proposition 1.6, 1b). On pourrait aussi appliquer directement le corollaire 1.7 et la compacité dans $\mathrm{SL}_{n}^{\rho}(\mathbb{Z}) \backslash P_{n}^{\rho}$.

Remarque 2.4. - D'après la proposition 2.5, 6), tout sous-ensemble $S_{A}$ (avec $A \in P_{n}^{\rho}$ ) est inclus dans une configuration maximale $S_{B}$ avec $B$ parfait dans $P_{n}^{\rho}$. Les classes qui apparaissent au voisinage de $B$ sont clairement en bijection avec les faces de dimension arbitraire du convexe engendré dans $T_{B} P_{n}^{\rho}$ par les gradients «minimaux » relatifs. D'où la possibilité, pour $\rho$ quelconque, de décrire toutes les classes minimales de $P_{n}^{\rho}$ à partir de ses points parfaits (donnés par l'algorithme de Voronoï).

Note 2.5. - La finitude des classes pour $\Pi$ trivial est classique (voir [11, prop. 3.2]) ainsi que la finitude des classes de П-réseaux dont les vecteurs minimaux engendrent $\mathbb{R}^{n}$ (voir [28, XI, th. 9.4], avec une preuve entièrement combinatoire basée sur l'inégalité d'Hermite [28, II théorème 2.1]. L'assertion 4) de la proposition 2.5 est à rapprocher de [28, XI, prop. 9.3]. Dans le cas usuel ( $\Pi$ trivial) la caractérisation variationnelle (proposition $2.5,3$ ) et la finitude des réseaux faiblement eutactiques sont établies dans [11] (théorème 3.4 et 3.5). L'assertion 2) du corollaire 2.6 se déduit aussi du cas usuel compte tenu de la finitude des fibres de $\mathrm{SL}_{n}^{\rho}(\mathbb{Z}) \backslash P_{n}^{\rho} \rightarrow \mathrm{SL}_{n}(\mathbb{Z}) \backslash P_{n}$; elle implique (via la remarque 2.6) les trois théorèmes de finitude de [26, p. 170]. Noter enfin que les formes faiblement eutactiques sont algébriques ( $c f$. [11, th. 4.1]), ce qui découle immédiatement du corollaire 1.12,2).

2.5. Familles de réseaux avec action d'un groupe fini. - Soit $V$ une sous-variété connexe, complète et totalement géodésique de l'espace symétrique riemannien $P_{n}$. Les réseaux paramétrés par $V$ constituent du point de vue 
géométrique une bonne famille car les fonctions longueur sont encore convexes en restriction à $V$. Posons

$$
G_{V}=\left\{P \in \mathrm{GL}_{n}(\mathbb{R}) ; P \cdot V=V\right\},
$$

sous-groupe de Lie de $\mathrm{GL}_{n}(\mathbb{R})$. Il est géométriquement clair que $V$ est stable par toute symétrie centrale $s_{A}$ centrée en $A \in V: V$ est donc aussi un espace symétrique, à courbure négative ou nulle. Soit $T_{V}$ l'adhérence du sous-groupe engendré par toutes les transvections $t_{A B}=s_{A} \circ s_{B}$ avec $A$ et $B$ dans $V$ $\left(T_{V} \subset G_{V} \cap \mathrm{SL}_{n}(\mathbb{R})\right)$. Dans la pratique il n'est pas toujours commode de déterminer $G_{V}$, mais on peut souvent exhiber un sous-groupe de Lie $G$ de $G_{V}$ qui contient $T_{V}$ (voir un exemple important au $\S 2.7$, proposition $2.11,1$ ). Pour la suite, on fixe un tel sous-groupe $G$ qui sera nécessairement transitif sur $V$.

$\grave{A}$ cette situation, on ajoute maintenant l'action d'un groupe fini $\Pi$, i.e. on fixe une représentation intégrale $\rho: \Pi \rightarrow G \cap \mathrm{GL}_{n}(\mathbb{Z})$. Soit $V^{\rho}$ l'ensemble des formes $\rho$-invariantes de $V$ :

$$
V^{\rho}=\left\{A \in V ; \rho(\pi) A \rho(\pi)^{t}=A, \forall \pi \in \Pi\right\} .
$$

On note $G^{\rho}(\mathbb{R})$ le commutant de $\rho$ dans $G \cap \mathrm{SL}_{n}(\mathbb{R})$ et on pose

$$
G^{\rho}(\mathbb{Z})=G^{\rho}(\mathbb{R}) \cap \mathrm{SL}_{n}(\mathbb{Z}) .
$$

Proposition 2.7. - 1) (propriétés géométriques) L'ensemble $V^{\rho}$ est une sous-variété connexe non vide, complète et totalement géodésique de $V$; c'est aussi un espace symétrique.

2) (propriétés algébriques) La composante neutre du commutant $G^{\rho}(\mathbb{R})$ agit transitivement sur $V^{\rho}$.

3) (propriétés topologiques) On suppose qu'il existe un sous-groupe de Lie $H$ de $G$ contenant $T_{V}$ et tel que $H \cap \mathrm{SL}_{n}(\mathbb{R})$ soit pseudo-algébrique (définition 2.1). Alors l'application $G^{\rho}(\mathbb{Z}) \backslash V^{\rho} \rightarrow \mathrm{SL}_{n}(\mathbb{Z}) \backslash P_{n}$ est propre et on a un critère de Mahler pour $V^{\rho}$ modulo $G^{\rho}(\mathbb{Z})$.

Preuve. - 1) Défini comme lieu des points fixes d'un groupe fini d'isométries, $V^{\rho}$ est une sous-variété complète totalement géodésique de $V$. Elle est non vide par le théorème de point fixe d'Élie Cartan, connexe par unicité des géodésiques dans $V$ et évidemment stable par les symétries centrées en ses points.

2) Pour $A \in V^{\rho}$ et $\pi \in \Pi$, on a $\rho(\pi) s_{A} \rho(\pi)^{-1}=s_{A}$ car $\rho(\pi)$ fixe $A$, donc $s_{A}$ commute avec $\rho$. Par suite $t_{A B}\left(A, B \in V^{\rho}\right)$ appartient au commutant $G^{\rho}(\mathbb{R})$ (on rappelle que par hypothèse $t_{A B} \in G$ ). Comme les transvections font partie de sous-groupes à un paramètre (ou parce que $V^{\rho}$ est connexe), on conclut que la composante neutre de $G^{\rho}(\mathbb{R})$ est transitive sur $V^{\rho}$.

3) Vérifions d'abord que $H^{\rho}(\mathbb{R})=H \cap G^{\rho}(\mathbb{R})$ est pseudo-algébrique. Soient $\varphi, v$ et $L \subset \mathbb{R}^{N}$ comme dans la définition 2.1 , et soit $R_{1}, \ldots, R_{r}$ une 
famille génératrice de $\rho(\Pi)$. Posons

$$
\psi(P) \cdot\left(x, M_{1}, \ldots, M_{r}\right)=\left(\varphi(P) \cdot x, P M_{1} P^{-1}, \ldots, P M_{r} P^{-1}\right)
$$

où $x \in \mathbb{C}^{N}$ et $M_{j} \in M_{n}(\mathbb{C})(j=1, \ldots, r)$. Le réseau $M=L \times M_{n}(\mathbb{Z})^{r}$ est stable par $\psi\left(\mathrm{SL}_{n}(\mathbb{Z})\right)$, et pour $w=\left(v, R_{1}, \ldots, R_{r}\right) \in M$ on a

$$
H^{\rho}(\mathbb{R})=\left\{P \in \mathrm{SL}_{n}(\mathbb{R}) ; \psi(P) \cdot w=w\right\} .
$$

Mais d'après la preuve de 2 ), $H^{\rho}(\mathbb{R})$ est transitif sur $V^{\rho}$, donc (proposition 2.1) $\left(H^{\rho}(\mathbb{R}) \cap \mathrm{SL}_{n}(\mathbb{Z}) \backslash V^{\rho} \rightarrow \mathrm{SL}_{n}(\mathbb{Z}) \backslash P_{n}\right.$ est propre, d'où l'assertion 3$)$.

Remarque 2.6. - Soit $N_{G}^{\rho}(\mathbb{Z})\left(\right.$ resp. $B_{G}^{\rho}(\mathbb{Z})$ ) le sous-groupe de $G \cap \mathrm{GL}_{n}(\mathbb{Z})$ des éléments qui laissent stable (resp. qui fixent point par point) $V^{\rho}$. En fait, $N_{G}^{\rho}(\mathbb{Z})$ est le normalisateur du « groupe de Bravais » $B_{G}^{\rho}(\mathbb{Z})$ dans $G \cap \mathrm{GL}_{n}(\mathbb{Z})$ et contient évidemment le commutant $G^{\rho}(\mathbb{Z})$. Tous les théorèmes de finitude ou de compacité dans $V^{\rho}$ modulo $G^{\rho}(\mathbb{Z})$ seront donc a fortiori valables modulo $N_{G}^{\rho}(\mathbb{Z})$.

Signalons enfin le comportement très simple des propriétés d'eutaxie :

Lemme 2.8. - Soit $D$ une partie non vide de $\mathbb{Z}^{n} \backslash\{0\}$ stable par $G^{t} \cap \mathrm{GL}_{n}(\mathbb{Z})$. Alors l'eutaxie pour $\mu^{D}$ relativement à $V^{\rho}$ est équivalente à l'eutaxie relativement à $V$. Il en est de même pour l'eutaxie faible et pour la semi-eutaxie.

Preuve. - Si $A \in V^{\rho}$, la représentation $\rho^{\vee}$ à valeurs dans $G^{t} \cap \mathrm{GL}_{n}(\mathbb{Z})$ laisse stable l'ensemble $S_{A} \subset D$ des vecteurs minimaux de $A$ pour $\mu^{D}$ ainsi que les gradients $X_{s}$ de $f_{s \mid V}$ pour $s \in S_{A}$. Il suffit donc d'appliquer le lemme 1.2. Noter que le gradient $X_{s}^{\rho}$ de $f_{s \mid V^{\rho}}$ est donné par

$$
X_{s}^{\rho}(A)=\frac{1}{|\Pi|} \sum_{\pi \in \Pi} X_{\rho^{\vee}(\pi)(s)}(A) \quad\left(A \in V^{\rho}\right) .
$$

Remarque 2.7. - Le théorème de Voronoï ne passe généralement pas à la situation équivariante (comme c'est le cas pour $V=P_{n}$ ), même dans les meilleurs cas. Voici un exemple avec les réseaux symplectiques paramétrés par l'espace de Siegel $\mathfrak{H}_{g}$ (voir $[6]$ pour les détails). Soit $\rho: \mathbb{Z} / 2 \mathbb{Z} \rightarrow \operatorname{Sp}(2 g, \mathbb{Z})$ définie par $\rho(1)=\left(\begin{array}{cc}I_{p, q} & 0 \\ 0 & I_{p, q}\end{array}\right)$ où $I_{p, q}=\left(\begin{array}{cc}I_{p} & 0 \\ 0 & -I_{q}\end{array}\right)$ avec $p+q=g$ et $p \leq q$. Dans $\mathfrak{H}_{g}$, le lieu des points fixes est $\mathfrak{H}_{g}^{\rho}=\mathfrak{H}_{p} \times \mathfrak{H}_{q}$. S'il existe $\left(\tau_{1}^{0}, \tau_{2}^{0}\right)$ avec $\tau_{1}^{0}$ extrême dans $\mathfrak{H}_{p}$ et $\mu\left(\tau_{1}^{0}\right)<\mu\left(\tau_{2}^{0}\right)$, alors tout point $\left(\tau_{1}^{0}, \tau_{2}\right)$ avec $\tau_{2}$ voisin de $\tau_{2}^{0}$ est extrême dans $\mathfrak{H}_{g}^{\rho}$. Il est facile de trouver de tels points : par exemple $A_{2} \times D_{4}$ dans $\mathfrak{H}_{1} \times \mathfrak{H}_{2}$. 
2.6. Réseaux hermitiens complexes et quaternioniens. - Les réseaux possédant des structures algébriques particulières (réseaux symplectiques, orthogonaux,...) sont souvent paramétrés par des espaces symétriques et se rangent d'abord suivant trois grandes familles : réseaux usuels, hermitiens complexes ou hermitiens quaternioniens. Après avoir fixé quelques notations, nous exprimons de façon unifiée les diverses quantités attachées à ces réseaux (longueurs, gradients, ...).

Soit $\boldsymbol{k}$ le corps $\mathbb{R}, \mathbb{C}$ ou $\mathbb{H}=\mathbb{R} \oplus i \mathbb{R} \oplus j \mathbb{R} \oplus k \mathbb{R}\left(i^{2}=j^{2}=k^{2}=-1\right.$, $i j=-j i=k)$. Pour $Z \in M_{p, q}(\boldsymbol{k})$, on pose $Z^{*}=\left(Z^{\sigma}\right)^{t} \in M_{q, p}(\boldsymbol{k})$ où $\sigma$ désigne la conjugaison usuelle pour $\mathbb{C}$ ou $\mathbb{H}$ et l'identité pour $\mathbb{R}$ (anti-automorphisme involutif de $\boldsymbol{k}$ ). Remarquer que

$$
(Z W)^{*}=W^{*} Z^{*} \quad\left(Z \in M_{p, q}(\boldsymbol{k}), W \in M_{q, r}(\boldsymbol{k})\right) .
$$

On considère $\boldsymbol{k}^{m}\left(m \in \mathbb{N}^{*}\right)$ comme $\boldsymbol{k}$-espace vectoriel $\grave{a}$ droite (i.e. à gauche si $\boldsymbol{k}=\mathbb{R}$ ou $\mathbb{C}$ ), de sorte que la multiplication à gauche par les matrices définit un isomorphisme de $M_{p, q}(\boldsymbol{k})$ sur $\operatorname{End}_{\boldsymbol{k}}\left(\boldsymbol{k}^{q}, \boldsymbol{k}^{p}\right)$. L'espace $\boldsymbol{k}^{m}$ sera muni de la forme $\sigma$-sesquilinéaire hermitienne définie positive $h^{\sigma}$ :

$$
h^{\sigma}(x, y)=\langle x, y\rangle=x^{*} y \quad\left(x, y \in \boldsymbol{k}^{m}\right) .
$$

Elle induit une structure euclidienne sur l'espace réel sous-jacent à $\boldsymbol{k}^{m}$ et, si $\boldsymbol{k}=\mathbb{H}$, une structure hermitienne complexe sur le $\mathbb{R}[j]$-espace vectoriel $\boldsymbol{k}^{m}$. Le groupe spécial unitaire de $h^{\sigma}$ sera noté simplement $\mathrm{SU}_{m}(\boldsymbol{k})$. C'est le groupe des matrices $A \in \mathrm{SL}_{m}(\boldsymbol{k})$ telles que $A A^{*}=I_{m}$ (matrice identité) (i.e. $\mathrm{SO}_{m}(\mathbb{R})$ si $\boldsymbol{k}=\mathbb{R}$ et $\mathrm{SU}_{m}(\mathbb{C})$ si $\left.\boldsymbol{k}=\mathbb{C}\right)$. Noter que $\mathrm{SU}_{m}(\mathbb{H})=\mathrm{U}_{m}(\mathbb{H})$.

Soit $\mathcal{O}$ l'anneau $\mathbb{Z}, \mathbb{Z}[i]$ ou $\mathbb{Z}[i, j, k]$ dans $\mathbb{R}, \mathbb{C}$ ou $\mathbb{H}$. Les $\mathcal{O}$-modules à droite engendrés par les $\boldsymbol{k}$-bases de $\boldsymbol{k}^{m}$ seront appelés $\mathcal{O}$-réseaux (ou réseaux) marqués de $\boldsymbol{k}^{m}$. Après transformation par une homothétie centrale, tout réseau marqué se met sous la forme $\Lambda=P^{*} \mathcal{O}^{m}$ avec $P \in \mathrm{SL}_{m}(\boldsymbol{k})$. En particulier $\Lambda$ est de covolume 1 pour la structure euclidienne sous-jacente. En associant à $P^{*} \mathcal{O}^{m}$ sa matrice de Gram $P P^{*}$, on définit une bijection entre l'espace des matrices de Gram

$$
P_{m}^{k}=\left\{A \in \mathrm{SL}_{m}(\boldsymbol{k}) ; A=A^{*}, A \text { définie positive }\right\}
$$

et l'ensemble des réseaux de $\boldsymbol{k}^{m}$ marqués par une base de déterminant 1 , à $h^{\sigma}$ isométrie unimodulaire près, usuels si $\boldsymbol{k}=\mathbb{R}$, hermitiens complexes si $\boldsymbol{k}=\mathbb{C}$ ou hermitiens quaternioniens si $\boldsymbol{k}=\mathbb{H}$. L'action de $\mathrm{SL}_{m}(\boldsymbol{k})$ sur $P_{m}^{\boldsymbol{k}}$ par $P \cdot A=P A P^{*}$ donne que $P_{m}^{\boldsymbol{k}} \simeq \mathrm{SL}_{m}(\boldsymbol{k}) / \mathrm{SU}_{m}(\boldsymbol{k})$. Observons enfin que l'on a un critère de Mahler dans l'espace des réseaux $\mathrm{SL}_{m}(\mathcal{O}) \backslash P_{m}^{k}$ (remarque 2.8 et $\S 2.4)$.

Bien que l'on puisse toujours se ramener à des sous-variétés de $P_{n}=P_{n}^{\mathbb{R}}$ (remarque 2.8), il est souvent utile, pour ne pas augmenter la taille des matrices, de travailler directement dans $P_{m}^{k}$. À cet effet on munit $P_{m}^{k}$ d'une structure d'espace symétrique riemannien grâce à la métrique réelle $\mathrm{SL}_{m}(\boldsymbol{k})$-invariante 
généralisant (2.4) :

$$
\langle X, Y\rangle_{A}=\operatorname{Tr}^{h}\left(A^{-1} X A^{-1} Y\right) \quad\left(A \in P_{m}^{k}, X, Y \in T_{A} P_{m}^{k}\right) .
$$

où $\operatorname{Tr}^{h} M=\frac{1}{2} \operatorname{Tr}\left(M+M^{*}\right)$ pour $M \in M_{m}(\boldsymbol{k})$. Pour $k=\mathbb{R}$ ou $\mathbb{C}$, on peut remplacer $\operatorname{Tr}^{h}$ par $\operatorname{Tr}$ dans (2.9). Si $H_{m}(\boldsymbol{k})$ désigne l'espace des matrices $h^{\sigma}$ hermitiennes, l'espace tangent à $P_{m}^{k}$ au point $A$ est donné par

$$
T_{A} P_{m}^{k}=\left\{X \in H_{m}(\boldsymbol{k}) ; \operatorname{Tr}^{h}\left(A^{-1} X\right)=0\right\} .
$$

Les fonctions longueur $f_{u}$ et leurs gradients $\nabla_{u}$ pour (2.9) s'expriment comme dans le cas réel :

$$
A\{u\}=f_{u}(A)=u^{*} A u, \quad \nabla_{u}(A)=A u u^{*} A-\frac{A\{u\}}{m} A
$$

avec $A \in P_{m}^{k}$ et $u \in \boldsymbol{k}^{m}$. À noter que si $\boldsymbol{k}=\mathbb{H}$, la trace n'a pas les propriétés usuelles (notamment invariance par conjugaison), mais on a $\operatorname{Tr}^{h} A B=\operatorname{Tr}^{h} B A$ (si $A B$ et $B A$ sont définies) que l'on déduit par exemple de (2.12).

Posons $\mathbb{L}=\mathbb{R}[j]$ si $\boldsymbol{k}=\mathbb{H}$ et $\mathbb{L}=\mathbb{R}$ si $\boldsymbol{k}=\mathbb{R}[i]$, et soit $\left(e_{\alpha}\right)_{1 \leq \alpha \leq m}$ la base canonique de $\boldsymbol{k}^{m}$. En considérant $\boldsymbol{k}^{m}(\boldsymbol{k}=\mathbb{H}$ ou $\mathbb{R}[i])$ comme espace vectoriel à droite sur $\mathbb{L}$ de base $\left(e_{1}, \ldots, e_{m}, e_{1} i, \ldots, e_{m} i\right)$, on obtient un plongement $\theta_{\boldsymbol{k}}^{\mathbb{L}}$ de $M_{p, q}(\boldsymbol{k})$ dans $M_{2 p, 2 q}(\mathbb{L})$ défini par

$$
\theta_{\boldsymbol{k}}^{\mathbb{L}}(A+i B)=\left(\begin{array}{cc}
A-B^{\sigma} \\
B & A^{\sigma}
\end{array}\right) \quad\left(A, B \in M_{p, q}(\mathbb{L})\right)
$$

Il est immédiat de voir que $\theta_{\boldsymbol{k}}^{\mathbb{L}}\left(Z^{*}\right)=\left(\theta_{\boldsymbol{k}}^{\mathbb{L}}(Z)\right)^{*}$ et que $\theta_{\boldsymbol{k}}^{\mathbb{L}}(Z W)=\theta_{\boldsymbol{k}}^{\mathbb{L}}(Z) \theta_{\boldsymbol{k}}^{\mathbb{L}}(W)$ si $Z W$ est défini. On vérifie également que $\operatorname{det} \theta_{\boldsymbol{k}}^{\mathbb{L}}(Z)=(\operatorname{det} Z)(\operatorname{det} Z)^{\sigma}$ pour $Z \in \mathrm{GL}_{m}(\boldsymbol{k})\left(\right.$ si $\left.\boldsymbol{k}=\mathbb{H}, \operatorname{det} Z \in \mathbb{H}^{\times} /\left[\mathbb{H}^{\times}, \mathbb{H}^{\times}\right] \simeq \mathbb{R}_{+}^{\times}\right)$ainsi qu'une propriété particulière pour les matrices hermitiennes $Z=Z^{*}$ :

$$
\operatorname{Tr} Z=\frac{1}{2} \operatorname{Tr}\left[\theta_{\boldsymbol{k}}^{\mathbb{L}}(Z)\right] \quad\left(Z \in H_{m}(\boldsymbol{k})\right) .
$$

Les images des plongements $\theta_{\mathbb{R}[i]}^{\mathbb{R}}, \theta_{\mathbb{H}}^{\mathbb{R}[j]}$ et $\theta_{\mathbb{H}}^{\mathbb{R}}=\theta_{\mathbb{R}[j]}^{\mathbb{R}} \circ \theta_{\mathbb{H}}^{\mathbb{R}[j]}\left(\right.$ de $M_{p, q}(\mathbb{H})$ dans $\left.M_{4 p, 4 q}(\mathbb{R})\right)$ sont évidemment caractérisées par des relations de commutation. Posons par exemple

$$
J_{m}=\left(\begin{array}{cc}
0 & I_{m} \\
-I_{m} & 0
\end{array}\right), \quad L_{m}=\left(\begin{array}{cc}
-J_{m} & 0 \\
0 & J_{m}
\end{array}\right) .
$$

Alors l'image de $\theta_{\mathbb{R}[i]}^{\mathbb{R}}$ est le commutant de $J_{m}$ et celle de $\theta_{\mathbb{H}}^{\mathbb{R}}$ le commutant de $J_{2 m}$ et $L_{m}$ (matrices des multiplication à droite par $-j$ et $i$ dans $\mathbb{H}^{m}$ ).

Remarque 2.8. - On obtient par $\theta_{k}^{\mathbb{L}}$ des plongements de $P_{m}^{\mathbb{C}}$ dans $P_{2 m}^{\mathbb{R}}$, et de $P_{m}^{\mathbb{H}}$ dans $P_{2 m}^{\mathbb{C}}$ et $P_{4 m}^{\mathbb{R}}$, ce qui revient à écrire les matrices de Gram des réseaux pour la structure euclidienne ou hermitienne complexe sous-jacente. Les images 
de $P_{m}^{\mathbb{C}}$ dans $P_{2 m}^{\mathbb{R}}$ et de $P_{m}^{\mathbb{H}}$ dans $P_{4 m}^{\mathbb{R}}$ sont les points fixes de l'action de sousgroupes finis de matrices entières, respectivement $\left\langle J_{m}\right\rangle$ et $\left\langle J_{2 m}, L_{m}\right\rangle$, et sont donc totalement géodésiques.

2.7. Autodualité : propriétés générales. - On reprend les notations du $\S$ 2.6. Dans la suite, $\tau$ désigne l'identité (id) de $\boldsymbol{k}$ ou la conjugaison $\sigma$ si $\boldsymbol{k}=\mathbb{C}$. L'espace $\boldsymbol{k}^{m}$ sera muni d'une forme $\sigma$-sesquilinéaire (si $\tau=\mathrm{id}$ ) ou bilinéaire complexe (si $\boldsymbol{k}=\mathbb{C}$ et $\tau=\sigma$ ) $b$ définie par

$$
b(x, y)=\left\langle x^{\tau}, K y\right\rangle=x^{\tau *} K y \quad\left(x, y \in \boldsymbol{k}^{m}\right) .
$$

On suppose de plus que $K$ est unitaire $\left(K \in \mathrm{U}_{m}(\boldsymbol{k})\right)$, ce qui entraîne que le groupe $\mathrm{U}_{b}(\boldsymbol{k})=\left\{P \in \mathrm{GL}_{m}(\boldsymbol{k}) ; P^{\tau *} K P=K\right\}$ est stable par l'opération $*$. Pour tout réseau $\Lambda$ de $\boldsymbol{k}^{m}$ on pose

$$
\Lambda^{* b}=\left\{y \in \boldsymbol{k}^{m} ; \forall z \in \Lambda,\langle z, y\rangle \in \mathcal{O}\right\}=K^{-1}\left(\Lambda^{*}\right)^{\tau}
$$

où $\Lambda^{*}$ est le dual usuel obtenu pour $b=\langle.,$.$\rangle . On dit que \Lambda$ est $b$-autodual si $\Lambda=\Lambda^{* b}$, i.e. $K \Lambda=\left(\Lambda^{*}\right)^{\tau}$, ce qui équivaut à dire que $b_{\mid \Lambda \times \Lambda}$ est à valeurs dans $\mathcal{O}$. Un tel réseau est évidemment isodual si $\tau=\mathrm{id}$, et isodual pour la structure euclidienne sous-jacente dans le cas bilinéaire complexe.

Dans le cas hermitien, tout réseau $b$-autodual s'écrit sous la forme

$$
\Lambda=P^{*} \mathcal{O}^{m} \quad \text { où } \quad P \in \mathrm{SL}_{m}(\boldsymbol{k}),
$$

après transformation par une homothétie de module 1 si $\boldsymbol{k}=\mathbb{C}$. Pour le cas bilinéaire sur $\mathbb{C}$, on pourra supposer, quitte à changer $K$ en $\alpha K$ (avec $|\alpha|=1$ ), qu'il existe un réseau $b$-autodual de la forme (2.14); à type fixé (voir ci-dessous), tous les réseaux $b$-autoduaux sont alors de cette forme. Remarquer que dans tous les cas $P^{\tau} K P^{*} \in \mathrm{GL}_{m}(\mathcal{O})$ (on peut utiliser (2.11) pour $\boldsymbol{k}=\mathbb{H}$ ).

Il est naturel de classer les réseaux $b$-autoduaux suivant leur type algébrique comme $\mathcal{O}$-modules sesquilinéaires ou bilinéaires (qui est évidemment localement constant). Par ailleurs, pour avoir une bonne description géométrique, il convient de considérer des réseaux marqués.

DÉfinition 2.2. - Soit $f: \mathcal{O}^{m} \times \mathcal{O}^{m} \rightarrow \mathcal{O}$ une forme $\sigma$-sesquilinéaire ou bilinéaire, et soit $f_{\boldsymbol{k}}$ son extension naturelle à $\boldsymbol{k}^{m} \times \boldsymbol{k}^{m}$. Un $\boldsymbol{k}$-isomorphisme unimodulaire de $f_{\boldsymbol{k}}$ sur $b$ (on suppose qu'il en existe) sera appelé réseau $b$ autodual marqué de type $f$.

Exemple 2.9. - Pour les réseaux symplectiques réels ou complexes il n'y a qu'un seul type. Pour les réseaux orthogonaux réels, il y a deux types : pair si $p-q$ est multiple de 8 , et impair (voir exemple 2.11). Concernant la finitude des types, voir plus généralement la fin de la preuve du lemme 2.19. 
Soit $F$ la matrice de $f$ dans la base canonique de $\mathcal{O}^{m}$ (on a nécessairement $F \in \mathrm{GL}_{m}(\mathcal{O})$ ). Tout réseau $b$-autodual marqué de type $f$ se ramène à la forme (2.14) avec $P \in \mathcal{P}_{f, b}=\left\{P \in \mathrm{SL}_{m}(\boldsymbol{k}) ; P^{\tau} K P^{*}=F\right\}$. Posons

$$
V_{f, b}=\left\{P P^{*} ; P \in \mathcal{P}_{f, b}\right\} \quad \text { et } \quad \Sigma_{b}=\mathrm{SU}_{b}(\boldsymbol{k}) \cdot I .
$$

Il est clair que $\mathcal{P}_{f, b}$ est une classe à droite modulo $\mathrm{SU}_{b}^{*}(\boldsymbol{k})=\mathrm{SU}_{b}(\boldsymbol{k})$ et une classe à gauche modulo $\mathrm{SU}_{f}^{*}(\boldsymbol{k})$ dans $\mathrm{SL}_{m}(\boldsymbol{k})$. Par suite, si $A_{0}=P_{0} P_{0}^{*} \in V_{f, b}$ $\left(P_{0} \in \mathcal{P}_{f, b}\right)$, on a

$$
V_{f, b}=P_{0} \Sigma_{b} P_{0}^{*}=\mathrm{SU}_{f}^{*}(\boldsymbol{k}) \cdot A_{0} .
$$

La description de $V_{f, b}$ avec $\mathrm{SU}_{b}(\boldsymbol{k})$ donne les propriétés géométriques (proposition 2.9) tandis que celle avec $\mathrm{SU}_{f}^{*}(\boldsymbol{k})$ donne les propriétés algébriques (proposition 2.11). Le quotient $\mathrm{SU}_{f}^{*}(\mathcal{O}) \backslash V_{f, b}$ s'identifie avec l'ensemble des réseaux $b$-autoduaux de type $f$ modulo l'action de $\mathrm{SU}_{b}(\boldsymbol{k}) \cap \mathrm{SU}_{m}(\boldsymbol{k})$.

Proposition 2.9. - 1) $V_{f, b}$ et $\Sigma_{b}$ sont des sous-variétés connexes, complètes et totalement géodésiques de $P_{m}^{k}$.

2) $\Sigma_{b}=\left\{B \in P_{m}^{k} ; B^{\tau} K B=K\right\}$ et $V_{f, b}=\left\{A \in P_{m}^{k} ; A^{\tau} F^{*-1} A=F\right\}$.

Lemme 2.10. - Soit $X$ une partie de $P_{m}^{\boldsymbol{k}}$ stable par toutes les symétries centrées en ses points (on dira que $X$ est symétrique). On suppose de plus que $X$ est fermée et qu'elle n'a qu'un nombre fini de composantes connexes. Alors $X$ est une sous-variété connexe, complète et totalement géodésique de $P_{m}^{k}$.

Preuve du lemme. - On peut supposer que $I \in X$. Soit $X^{0}$ la composante connexe de $I$ et soit $H$ l'adhérence dans $\mathrm{SL}_{m}(\boldsymbol{k})$ du sous-groupe engendré par les transvections $t_{A B}=s_{A} \circ s_{B}$ pour $A, B \in X^{0}\left(s_{A}(C)=A C^{-1} A, A\right.$ et $\left.C \in P_{m}^{k}\right)$. Il s'agit un sous-groupe de Lie réel de $\mathrm{SL}_{m}(\boldsymbol{k})$ stable par $*$ (conjugaison par $s_{I}$ ). De plus $H$ est connexe. En effet soit $H^{0}$ la composante neutre de $H$, ouverte dans $H$, et soient $A, B \in X^{0}$. Si $A$ et $B$ sont proches, $t_{A B} \in H^{0}$ («petite » transvection). Si $A$ et $B$ sont quelconques, puisque $X^{0}$ est connexe par chaîne, $t_{A B}$ s'écrit comme produit de « petites » transvections et donc appartient à $H^{0}$. Dans ces conditions, on sait que $H \cdot I$ est une sous-variété complète totalement géodésique (voir [20, p. 131]). Il en résulte que $X^{0}=H \cdot I$ : si $A \in X^{0}$, alors $t_{I A}(I) \in H \cdot I$, donc $A \in H \cdot I$.

Montrons pour conclure que $X$ est connexe. Considérons $B \in X \backslash X^{0}$ supposé non vide. La transvection $t=s_{B} \circ s_{I}$ laisse stable $X$ ainsi que la géodésique passant par $I$ et $B$. Il existe alors deux points distincts de l'orbite $\left\{t^{p}(I)\right\}_{p \in \mathbb{Z}}$ situés sur une même composante $t^{p}\left(X^{0}\right)$ totalement géodésique, ce qui contredit clairement l'unicité des géodésiques dans $P_{m}^{k}$.

Preuve de la proposition. - 1) Soit $G$ un sous-groupe de Lie réel de $\mathrm{SL}_{m}(\boldsymbol{k})$ stable par $*$ et qui n'a qu'un nombre fini de composantes connexes. Alors l'orbite $X=G \cdot I$ est symétrique (stabilité par $*$ ) et fermée sachant que $G^{0} \cdot I$ est 
une sous-variété complète totalement géodésique. Par conséquent (lemme 2.10) $X=G^{0} \cdot I=G \cdot I$. Le groupe $\mathrm{SU}_{b}(\boldsymbol{k})=\mathrm{SU}_{b}^{*}(\boldsymbol{k})$ est un ensemble algébrique réel, donc n'a qu'un nombre fini de composantes connexes (voir [13, th. 2.4.5]). Par suite $\Sigma_{b}=\mathrm{SU}_{b}^{0}(\boldsymbol{k}) \cdot I$, ce qui prouve 1$)$.

2) Posons $W_{b}=\left\{B \in P_{m}^{k} ; B^{\tau} K B=K\right\}$, partie fermée qui n'a qu'un nombre fini de composantes connexes (ensemble algébrique réel). Comme $W_{b}$ est symétrique, c'est une sous-variété connexe d'après le lemme 2.10 (la structure de sous-variété peut aussi s'obtenir directement avec le théorème du rang constant). Soit $\pi: \mathrm{SU}_{b}^{0}(\boldsymbol{k}) \rightarrow W_{b}$ définie par $\pi(P)=P P^{*}$. L'image de $\pi$ est fermée et vaut $\Sigma_{b}$ d'après l'assertion 1). Par ailleurs on a $T_{I} \mathrm{SU}_{b}^{0}(\boldsymbol{k})=$ $T_{I} W_{b} \oplus \operatorname{ker} \mathrm{d} \pi(I)$ (décomposer les matrices suivant leurs parties hermitienne et antihermitienne). Par conséquent $\pi$ est ouverte (submersion) et $\Sigma_{b}=W_{b}$. L'équation de $V_{f, b}$ se déduit alors de (2.15).

Remarque 2.10. - On peut voir que $\mathrm{U}_{f}(\boldsymbol{k})$ est stable par $*$ si et seulement si $F \in \mathrm{U}_{m}(\boldsymbol{k})$, ce qui revient à dire (proposition 2.9,2) que $V_{f, b}$ passe par $I$.

EXEMPLES 2.11 (réseaux et espaces symétriques irréductibles)

Tous les espaces symétriques irréductibles de type non compact et non exceptionnels, autres que $P_{m}^{\boldsymbol{k}}$, apparaissent naturellement comme espace $\Sigma_{b}$ de paramètres de réseaux $b$-autoduaux à type fixé :

- $b(x, y)=x^{*}\left(\begin{array}{cc}I_{p} & 0 \\ 0 & -I_{q}\end{array}\right) y$, forme hermitienne indéfinie de signature $(p, q)$, $\boldsymbol{k}=\mathbb{R}, \mathbb{C}$ ou $\mathbb{H}, \Sigma_{b}=\mathrm{SO}^{0}(p, q) / \mathrm{SO}(p) \times \mathrm{SO}(q), \mathrm{SU}(p, q) / \mathrm{S}\left(\mathrm{U}_{p} \times \mathrm{U}_{q}\right)$ ou $\operatorname{Sp}(p, q) / \operatorname{Sp}(p) \times \operatorname{Sp}(q)$ (réseaux orthogonaux);

- $b$ symplectique, $\boldsymbol{k}=\mathbb{R}$ ou $\mathbb{C}(\tau=\sigma), \Sigma_{b}=\mathrm{Sp}_{2 m}(\mathbb{R}) / \mathrm{U}(m)$ ou $\mathrm{Sp}_{2 m}(\mathbb{C}) / \mathrm{Sp}(m)$ (réseaux symplectiques);

- $b(x, y)=x^{t} y, \boldsymbol{k}=\mathbb{C}(\tau=\sigma), \Sigma_{b}=\mathrm{SO}_{m}(\mathbb{C}) / \mathrm{SO}_{m}(\mathbb{R})$

- $b(x, y)=x^{*} i y, \boldsymbol{k}=\mathbb{H}, \Sigma_{b}=\mathrm{SO}^{*}(2 m) / \mathrm{U}(m)$.

Pour les notations $\mathrm{U}(m)=\mathrm{U}_{m}(\mathbb{C}), \operatorname{Sp}(m)=\mathrm{U}_{m}(\mathbb{H})=\mathrm{SU}_{m}(\mathbb{H}), \operatorname{Sp}(p, q)$, $\mathrm{SO}^{*}(2 m)$, voir $[22$, p. 445]. La correspondance entre ces diverses notations est immédiate à partir de $M_{m}(\mathbb{H})=M_{m}(\mathbb{R}[j]) \oplus i M_{m}(\mathbb{R}[j])$. Toutes ces familles de réseaux vérifient le théorème de Voronoï, voir [1].

Proposition 2.11. - 1) $\mathrm{SU}_{f}^{*}(\boldsymbol{k})$ contient toutes les transvections $t_{A B}$ pour $A, B \in V_{f, b}$.

2) $\theta_{\boldsymbol{k}}^{\mathbb{R}}\left(\mathrm{SU}_{f}^{*}(\boldsymbol{k})\right)$ est pseudo-algébrique $\left(\theta_{\mathbb{R}}^{\mathbb{R}}\right.$ désigne l'identité de $\left.M_{m}(\mathbb{R})\right)$.

Preuve. - 1) On a $t_{A B}(C)=A B^{-1} \cdot C$ avec $A B^{-1} \in \mathrm{SU}_{f}^{*}(\boldsymbol{k})$ si $A, B \in V_{f, b}$.

2) Traitons par exemple le cas bilinéaire complexe $(\boldsymbol{k}=\mathbb{C}$ et $\tau=\sigma)$. Pour $Q$ appartenant à $\mathrm{SL}_{2 m}(\mathbb{R})$, on pose $\phi(Q)=\left(\begin{array}{cc}0 & I \\ I & 0\end{array}\right) Q\left(\begin{array}{ll}0 & I \\ I & 0\end{array}\right)$ en remarquant que l'on 
a $\theta_{\mathbb{C}}^{\mathbb{R}}\left(P^{\sigma}\right)=\phi\left(\theta_{\mathbb{C}}^{\mathbb{R}}(P)\right)$ si $P \in \mathrm{SL}_{m}(\mathbb{C})$. On prend donc

$$
\varphi(Q) \cdot(M, N)=\left(Q M Q^{-1}, \phi(Q) N Q^{t}\right),
$$

avec $\left.Q \in \mathrm{SL}_{2 m}(\mathbb{C}),(M, N) \in M_{2 m}(\mathbb{C})^{2}\right), L=M_{2 m}(\mathbb{Z})^{2}$ et $v=\left(J_{m}, \theta_{\mathbb{C}}^{\mathbb{R}}(F)\right.$, voir définition 2.1 et équation (2.13). Les autres cas sont aussi simples.

Par suite, si $\Pi$ est un groupe fini et si $G$ est un sous-groupe de Lie réel de $G_{V_{f, b}}$ contenant $\mathrm{SU}_{f}^{*}(\boldsymbol{k})$, on aura une bonne théorie équivariante pour les représentations $\rho: \Pi \rightarrow G \cap \mathrm{GL}_{m}(\mathcal{O})$ (proposition 2.7). Soit $D$ une partie non vide de $\mathcal{O}^{m} \backslash\{0\}$ stable par $G^{*} \cap \mathrm{GL}_{m}(\mathcal{O})$ et soit $\mu^{D}$ défini par $\mu^{D}(A)=$ $\inf _{u \in D} A\{u\}$, que l'on note simplement $\mu$ si $D=\mathcal{O}^{n} \backslash\{0\}$. Notons $G^{\rho}(\boldsymbol{k})$ le commutant de $\rho$ dans $G \cap \mathrm{SL}_{m}(\boldsymbol{k})$ et

$$
G^{\rho}(\mathcal{O})=G^{\rho}(\boldsymbol{k}) \cap \mathrm{SL}_{m}(\mathcal{O}) .
$$

Corollaire 2.12. - 1) On a un critère de Mahler pour $G^{\rho}(\mathcal{O}) \backslash V_{f, b}^{\rho}$ (i.e. l'inégalité $\mu \geq \epsilon>0$ définit un compact dans $\left.G^{\rho}(\mathcal{O}) \backslash V_{f, b}^{\rho}\right)$.

2) Les points parfaits ainsi que les points eutactiques non dégénérés pour $\mu^{D}$ relativement à $V_{f, b}^{\rho}$ sont algébriques sur $\mathbb{Q}$.

Preuve. - L'assertion 1) est la conséquence des propositions 2.7, 3) et 2.11. Sachant que $V_{f, b}$ (ainsi que $V_{f, b}^{\rho}$ ) est totalement géodésique et définie dans $P_{n}^{\mathbb{R}}$ $(n=m, 2 m$ ou $4 m)$ par des équations polynomiales à coefficients entiers (proposition 2.9 et remarque 2.8), l'assertion 2) résulte du corollaire 1.12.

Nous introduisons maintenant la notion de point et de classe isotropes. Soit $A \in V_{f, b}^{\rho}$ et soit $S=S_{A}$ pour $\mu^{D}$. On dira que $A$ est isotrope (pour $\mu^{D}$ ) et que $\mathcal{C}_{S}$ est une classe minimale isotrope de $V_{f, b}^{\rho}$ si le sous-espace de $\mathcal{O}^{m}$ (ou de $\boldsymbol{k}^{m}$ ) engendré par $S$ est totalement isotrope pour $f$.

Proposition 2.13 (finitude des classes non isotropes)

1) Soit $A \in V_{f, b}^{\rho}$ un point non isotrope pour $\mu^{D}$. Alors $\mu^{D}(A) \geq 1$.

2) Le nombre de classes minimales pour $\mu$ non isotropes de $V_{f, b}^{\rho}$ est fini modulo $G^{\rho}(\mathcal{O})$.

Preuve. - Comme $K$ est unitaire, d'après l'inégalité de Cauchy-Schwarz dans $\boldsymbol{k}^{m}$, on a

$$
|b(x, y)|^{2} \leq|x|^{2} \cdot|y|^{2} \quad\left(x, y \in \boldsymbol{k}^{m}\right) .
$$

Mais la forme $b$ est à valeurs dans $\mathcal{O}$ sur les réseaux $b$-autoduaux. Par suite, si $\mu^{D}(A)<1$, alors $S_{A}$ engendre un sous-espace totalement isotrope, d'où 1 ). L'assertion 2) résulte de 1) et du corollaire 2.12. 
2.8. Autodualité : le cas réflexif. - Nous supposerons désormais $m \geq 2$ et que la forme $b$ est réflexive (la relation $b(x, y)=0$ est symétrique en $x$ et $y$ ), ce qui comprend tous les cas intéressants, notamment les exemples 2.11. Il est facile de voir $(m \geq 2)$ que la réflexivité de $b$ équivaut à la condition suivante :

$$
K^{\tau} K=\kappa I \quad \text { avec } \kappa \text { central, }
$$

ou encore $K^{\tau}=\kappa K^{*}$. On a nécessairement $\kappa \kappa^{\sigma \tau}=1$ et $b(x, y)^{*}=\kappa^{-1} b(y, x)^{\tau}$ $\left(x, y \in \boldsymbol{k}^{m}\right)$. Si $\boldsymbol{k}=\mathbb{R}$ ou $\mathbb{H}$ (resp. $\boldsymbol{k}=\mathbb{C}$ et $\tau=\sigma$ ), alors $\kappa= \pm 1$ et $b$ est hermitienne ou antihermitienne (resp. symétrique ou antisymétrique). Si $\boldsymbol{k}=\mathbb{C}$ et $\tau=\mathrm{id}$, alors $|\kappa|^{2}=1$ et on peut multiplier $b$ pour la rendre hermitienne.

On confondra dans la suite toute matrice $M \in M_{m}(\boldsymbol{k})$ avec l'élément de $\mathcal{L}_{\boldsymbol{k}}\left(\boldsymbol{k}^{m}\right)$ qu'elle définit. L'application $\tau \circ M$ (semi-linéaire) sera notée $\tau M$, de sorte que $M^{\tau}=\tau M \tau$. Remarquer que $\tau K$ conserve l'orthogonalité et que (2.16) s'écrit $(\tau K)^{2}=\kappa I$.

Soit $H_{m}(\boldsymbol{k})$ le $\mathbb{R}$-espace vectoriel des matrices hermitiennes muni du produit euclidien défini positif $\langle X, Y\rangle_{I}=\operatorname{Tr}^{h}(X Y)$ (voir (2.9)). La conjugaison par $(\tau K)^{-1}$ définit une isométrie de $H_{m}(\boldsymbol{k})$ :

$$
\psi_{K}(X)=(\tau K)^{-1} X(\tau K)=K^{*} X^{\tau} K \quad\left(X \in H_{m}(\boldsymbol{k})\right) .
$$

D'après (2.16), $b$ est réflexive si et seulement si $\psi_{K}$ est involutive. On voit que $\Sigma_{b}=\{I\}$ équivaut à $\psi_{K}=\mathrm{Id}$, i.e. $\tau=$ id et $b$ proportionnelle, par un scalaire central de module 1 , au produit hermitien $\langle.,$.$\rangle (voir plus généralement le$ lemme $2.15,2$ ).

N.B. - Pour alléger les écritures, on ramènera très souvent l'étude d'un point $A=P P^{*}=P \cdot I \in V_{f, b}\left(P \in \mathcal{P}_{f, b}\right)$ à celle du point $I$ (point de vue du réseau $\left.\Lambda=P^{*} \mathcal{O}^{m}\right)$. Notons $\boldsymbol{k}_{A}^{m}$ l'espace $\boldsymbol{k}^{m}$ muni du produit scalaire hermitien $x^{*} A y\left(x, y \in \boldsymbol{k}^{m}\right)$. Considérons en outre une représentation $\rho$ à valeurs dans $G_{V_{f, b}} \cap \mathrm{GL}_{m}(\mathcal{O})$ et supposons $A \in V_{f, b}^{\rho}$ (i.e. $\rho^{\vee}=\rho^{*-1}$ est $A$-unitaire). On a un isomorphisme

$$
\left(\mathcal{O}^{m}, \boldsymbol{k}_{A}^{m}, f, \rho^{\vee}\right) \stackrel{P^{*}}{\longrightarrow}\left(\Lambda, \boldsymbol{k}_{I}^{m}, b, \varsigma\right)
$$

où $\varsigma=P^{*} \rho^{\vee} P^{*-1}=P^{-1} \rho P$ est unitaire et fixe $\Sigma_{b}$ dans $P_{m}^{k}$. Les gradients de $f_{P * s \mid \Sigma_{b}^{\varsigma}}$ en $I$ et de $f_{s \mid V_{f, b}^{\rho}}$ en $A$ se correspondent via l'action isométrique de $P$ $\operatorname{sur} P_{m}^{k}$.

Comme $\psi_{K}$ est involutive, la projection orthogonale de $H_{m}(\boldsymbol{k})$ sur l'espace tangent $T_{I} \Sigma_{b}=\operatorname{ker}\left(\psi_{K}+\mathrm{Id}\right)$ vaut simplement $\pi_{b}=\frac{1}{2}\left(\psi_{K}-\mathrm{Id}\right)$. On définit une application de $\boldsymbol{k}^{m}$ dans $T_{I} \Sigma_{b}$ par

$$
X_{u}=u u^{*}-K^{*} u^{\tau} u^{* \tau} K=2 \pi_{b}\left(u u^{*}\right) \quad\left(u \in \boldsymbol{k}^{m}, X_{u} \in T_{I} \Sigma_{b}\right) .
$$

BULlETIN DE LA SOCIÉTÉ MATHÉMATIQUE DE FRANCE 
Plus généralement, si $N$ est un sous-espace de $\boldsymbol{k}_{I}^{m}$, on note $P_{N}$ la matrice (dans la base canonique) de la projection orthogonale sur $N$ et on pose

$$
X_{N}=2 \pi_{b}\left(P_{N}\right)=P_{N}-P_{\tau K N} .
$$

On a $X_{N}=0$ si et seulement si $N$ est stable par $\tau K$; en particulier $X_{u}=0$ équivaut à $K u=u^{\tau} \alpha$ avec $\alpha \alpha^{\tau}=\kappa$. Noter aussi que $X_{u}=2 \nabla\left(f_{u \mid \Sigma_{b}}\right)(I)$ et que

$$
\frac{1}{2}\left\langle X, X_{u}\right\rangle_{I}=\left\langle X, u u^{*}\right\rangle_{I}=X\{u\} \quad\left(X \in T_{I} \Sigma_{b}, u \in \boldsymbol{k}^{m}\right) .
$$

Dans la suite on note :

- $\mathrm{ML}_{m}(\boldsymbol{k})$ le sous-groupe des $P$ appartenant à $\mathrm{GL}_{m}(\boldsymbol{k})$ tels que $|\operatorname{det}(P)|=1$ $\left(\mathrm{ML}_{m}(\mathbb{H})=\mathrm{SL}_{m}(\mathbb{H})\right)$,

- $\mathrm{MU}_{f}(\boldsymbol{k})$ le groupe des similitudes unitaires relatives à $f$ dont le multiplicateur (élément central) est de module 1.

Proposition 2.14 (stabilisateur de $V_{f, b}$ ). - $\mathrm{MU}_{f}^{*}(\boldsymbol{k})$ (resp. $\mathrm{MU}_{b}(\boldsymbol{k})$ ) est le stabilisateur de $V_{f, b}$ (resp. de $\left.\Sigma_{b}\right)$ pour l'action de $\mathrm{ML}_{m}(\boldsymbol{k})$ sur $P_{m}^{k}$.

Preuve. - Soit $P \in \mathrm{ML}_{m}(\boldsymbol{k})$ fixant $\Sigma_{b}$. Comme $\mathrm{SU}_{b}(\boldsymbol{k})$ est transitif sur $\Sigma_{b}$ on peut supposer que $P \cdot I=I$, i.e. $P \in \mathrm{U}_{m}(\boldsymbol{k})$. L'action isométrique de $P$ sur $T_{I} P_{\boldsymbol{k}}^{m}$ fixe $T_{I} \Sigma_{b}$, donc commute avec $\pi_{b}$. Par suite on a $P X_{u} P^{*}=X_{P u}$ pour tout $u \in \boldsymbol{k}^{m}$, c'est-à-dire $P K^{*} u^{\tau} u^{* \tau} K P^{*}=K^{*} P^{\tau} u^{\tau} u^{* \tau} P^{* \tau} K$, d'où l'on déduit que $P^{* \tau} K P K^{*} u^{\tau}=u^{\tau} \alpha_{u}\left(\alpha_{u} \in \boldsymbol{k}\right)$, puis $(m \geq 2)$ que $P^{* \tau} K P K^{*}=\alpha I$ avec $\alpha$ central de module 1 . Inversement il est clair que $\operatorname{MU}_{b}(\boldsymbol{k})$ stabilise $\Sigma_{b}$. Enfin, les groupes $\mathrm{MU}_{f}^{*}(\boldsymbol{k})$ et $\mathrm{MU}_{b}^{*}(\boldsymbol{k})=\mathrm{MU}_{b}(\boldsymbol{k})$ étant conjugués par tout élément de $\mathcal{P}_{f, b}$, on a aussi l'assertion sur $V_{f, b}$.

Considérons maintenant un sous-groupe de Lie $G$ de $\mathrm{MU}_{f}^{*}(\boldsymbol{k})$ contenant $\mathrm{SU}_{f}^{*}(\boldsymbol{k})$ ainsi qu'une partie $D$ non vide de $\mathcal{O}^{m} \backslash\{0\}$ stable par $G^{*} \cap \mathrm{GL}_{m}(\mathcal{O})$. Bien sûr l'exemple principal est $D=\mathcal{O}^{m} \backslash\{0\}$ (invariant d'Hermite), mais il y a d'autres choix intéressants, en particulier si $G=U_{f}^{*}(\boldsymbol{k})$ et si $f$ représente l'entier $\xi, D=\left\{u \in \mathcal{O}^{m} \backslash\{0\} ; f(u, u)=\xi\right\}$ ([4]).

THÉORÈme 1 (non isotropie des points faiblement eutactiques)

1) Tout point $A \in V_{f, b}^{\rho}$ faiblement eutactique pour $\mu^{D}$ est non isotrope (donc $\left.\mu^{D}(A) \geq 1\right)$.

2) Ici $D=\mathcal{O}^{m} \backslash\{0\}$. Modulo l'action de $G^{\rho}(\mathcal{O})$, il n'y a dans $V_{f, b}^{\rho} q u$ 'un nombre fini:

2a) de classes minimales contenant un point faiblement eutactique (par exemple extrême ou eutactique),

2b) de points parfaits,

2c) de points eutactiques non dégénérés. 
En particulier le nombre de points strictement extrêmes (i.e. parfaits et eutactiques) est fini modulo $G^{\rho}(\mathcal{O})$.

Preuve. - 1) On peut supposer que $\rho$ est triviale (lemme 2.8) et on se ramène par (2.17) au point $I \in \Sigma_{b}$. Soit $M$ le sous-espace de $\boldsymbol{k}^{m}$ engendré par l'ensemble des vecteurs minimaux. Si $M$ est totalement isotrope pour $b$, on a la décomposition orthogonale

$$
\boldsymbol{k}^{m}=M \oplus\left(M^{\perp} \cap M^{\perp b}\right) \oplus\left(M^{\perp b}\right)^{\perp} .
$$

On vérifie que $\tau K$ permute $M$ et $L=\left(M^{\perp b}\right)^{\perp}$ (et conserve $M^{\perp} \cap M^{\perp b}$ ). Par suite $X_{M}=P_{M}-P_{L} \in T_{I} \Sigma_{b}$ (voir (2.18)). D'après (2.19), on a $\left\langle X_{M}, X_{t}\right\rangle_{I}=$ $2 \mu(A) \neq 0$ pour tout vecteur minimal $t$ du réseau, ce qui contredit l'eutaxie faible (lemme 1.1, 4).

2) On déduit de l'assertion 1) la finitude des classes « faiblement eutactiques » (proposition 2.13) ainsi que celle des points eutactiques non dégénérés (proposition 1.6, 2), unicité). Comme $V_{f, b}^{\rho}$ est définie dans $P_{n}^{\mathbb{R}}(n=m, 2 m$ ou $4 m)$ par des équations algébriques à coefficients entiers (proposition 2.9, 2), la finitude des points parfaits résulte de $2 \mathrm{a}$ ) et du corollaire $1.12,1)$.

Note 2.12. - L'assertion 2c) est à comparer avec la finitude, pour les réseaux isoduaux réels et $\Pi$ trivial, des points eutactiques avec $\operatorname{rang}\left(S_{A}\right)$ maximal, $[10$, cor. 6.9]. La non isotropie est une condition bien plus faible; il existe par exemple un réseau orthogonal eutactique et non dégénéré avec $\operatorname{rang}\left(S_{A}\right)=1$ (exemple 2.15).

Soit $A=P P^{*} \in V_{f, b}^{\rho}$ où $P \in \mathcal{P}_{f, b}$. Dans les énoncés qui suivent, on notera toujours $M$ (resp. $M_{A}$ ) le sous-espace de $\boldsymbol{k}^{m}$ engendré (ou ce qui revient au même $\mathbb{R}$-engendré) par les vecteurs minimaux de $\Lambda=P^{*} \mathcal{O}^{m}$ (resp. de $A$ ) et $\varsigma$ la représentation à valeurs dans $\mathrm{U}_{m}(\boldsymbol{k}) \cap \mathrm{MU}_{b}(\boldsymbol{k})$ donnée par (2.17). Évidemment $M$ et $M^{\perp}$ sont stables par $\varsigma$ ( $\varsigma$-modules) car $S_{A} \subset D$ est stable par $\rho^{\vee}$.

DÉfinition 2.3. - Un sous-module $N$ du $\varsigma$-module $\boldsymbol{k}^{m}$ est totalement stable par une application $\varphi$ si tout sous-module de $N$ est stable par $\varphi$.

Lemme 2.15. - 1) Soit $N$ un sous-module de $\boldsymbol{k}^{m}$. Alors $N$ est totalement stable par $\tau K$ si et seulement si tout $\varsigma$-endomorphisme hermitien qui laisse stable $N$ commute avec $\tau K$.

2) La variété $V_{f, b}^{\rho}$ est réduite à un point si et seulement si le $\varsigma$-module $\boldsymbol{k}^{m}$ est totalement stable par $\tau K$.

Preuve. - 1) Supposons $N$ totalement stable par $\tau K$ et soit $X$ appartenant à $\mathcal{L}_{\varsigma}\left(\boldsymbol{k}^{m}\right) \cap H_{m}(\boldsymbol{k})$, où $\mathcal{L}_{\varsigma}\left(\boldsymbol{k}^{m}\right)$ désigne l'anneau des endomorphismes du $\varsigma$ module $\boldsymbol{k}^{m}$. Les composants isotypiques de $N$ étant stables par $X$ et $\tau K$, on peut supposer que $N$ est isotypique. Soit $S$ un sous-module simple de $N$ (type de $N$ ). L'élément de $\mathcal{L}_{\varsigma}(N)$ induit par $X$ sera encore noté $X$. On sait que 
$\Omega=\mathcal{L}_{\varsigma}(S)$ est un corps (lemme de Schur) et que la composition des applications donne à droite une structure de $\Omega$-espace vectoriel (à droite) sur $W=\mathcal{L}_{\varsigma}(S, N)$, et à gauche un isomorphisme d'anneaux du commutant $\mathcal{L}_{\varsigma}(N)$ sur $\mathcal{L}_{\Omega}(W)$. De plus $\mathcal{L}_{\varsigma}(S, \cdot)$ est une correspondance bijective entre les sous-modules de $N$ et les sous-espaces vectoriels de $W(c f .[14, \mathrm{Alg} ., \mathrm{ch} .8, \S 4.4])$. Sachant que $\varsigma$ est à valeurs dans $\mathrm{U}_{m}(\boldsymbol{k}) \cap \mathrm{MU}_{b}(\boldsymbol{k})$ et que $\tau K$ stabilise les sous-modules de $N$, on vérifie que la conjugaison par $\tau K$ induit une application semi-linéaire $\Phi$ de $W$ dans $W$ stabilisant chaque sous-espace vectoriel. De même $\tau K X(\tau K)^{-1}$ est dans $\mathcal{L}_{\varsigma}(N)$.

Si $\operatorname{dim}_{\Omega} W \geq 2$, il existe $\lambda \in \Omega$ tel que $\Phi(w)=w \circ \lambda$ pour tout $w \in W$ ( $\Phi$ stabilise chaque droite). Mais $\Phi$ fixe l'injection de $S$ dans $N$, donc $\lambda=1$. Pour tout $w \in W$, on a $X \circ w \in W$ et $\tau K X(\tau K)^{-1} \circ w=\Phi(X \circ w)=X \circ w$, d'où $\tau K X(\tau K)^{-1}=X$.

Reste le cas où $\operatorname{dim}_{\Omega} W=1$, i.e. $N=S$. Le corps $\Omega=W$ est muni de l'automorphisme continu $\Phi$, de l'anti-automorphisme involutif continu $\xi$ induit par $*$, et on a $X \in \Omega^{\xi}$. On peut écarter le cas évident où $\Omega$ est isomorphe à $\mathbb{R}$. Alors $\Omega$ contient un élément de carré -1 qui ne peut être hermitien car non positif, donc $\xi$ est non trivial. Si $\Omega \simeq \mathbb{C}$, $\xi$ est la conjugaison et $\Phi$ (identité ou conjugaison) fixe chaque élément de $\Omega^{\xi}$. Enfin si $\Omega \simeq \mathbb{H}$, $\xi$ est conjugué à la conjugaison (anti-automorphisme involutif) et $\operatorname{dim}_{\mathbb{R}} \Omega^{\xi}=1$; mais $\Omega^{\xi}$ contient les homothéties centrales, c'est donc le centre de $\Omega$ et il est fixe point par point par $\Phi$.

Réciproquement, soit $L$ un sous-module de $N$. Alors la projection $P_{L}$ commute avec $\tau K$, i.e. $L$ est stable par $\tau K$.

2) L'espace tangent $T_{I} \Sigma_{b}^{\varsigma}$ est réduit à $\{0\}$ si et seulement si tout élément de $H_{m}(\boldsymbol{k}) \cap \mathcal{L}_{\varsigma}\left(\boldsymbol{k}^{m}\right)$ commute avec $\tau K$. L'assertion 2) est donc conséquence de l'assertion 1).

ThÉORÈme 2 (critère de non dégénérescence). - Soit $\rho: \Pi \rightarrow G \cap \mathrm{GL}_{m}(\mathcal{O})$ et soit $A \in V_{f, b}^{\rho}$.

1) On suppose que $M^{\perp}$ est totalement stable par $\tau K$ (ou de façon équivalente que le $\rho^{\vee}$-module $M_{A}^{\perp_{A}}$ est totalement stable par $\left.\tau F^{\vee} A\right)$. Alors A est non dégénéré pour $\mu^{D}$.

2) Si A est eutactique pour $\mu^{D}$, alors $M^{\perp}=M^{\perp_{b}}$ (i.e. $M^{\perp}$ est stable par $\tau K)$ et $A$ est non dégénéré si et seulement si $M^{\perp}$ est totalement stable par $\tau K$.

Preuve. - 1) Soit $X \in T_{I} \Sigma_{b}^{\varsigma}$ nulle sur $M$. Comme $X$ est hermitienne, $X$ laisse stable le $\varsigma$-module $M^{\perp}$ donc (lemme 2.15,1) commute avec $\tau K$. De plus $X \in \operatorname{ker}\left(\psi_{K}+\mathrm{Id}\right)$, ce qui entraîne $X=0$ et on conclut que $A$ est non dégénéré (proposition 2.3). 
2) Pour établir $M^{\perp}=M^{\perp_{b}}$, on peut supposer $\rho$ triviale (lemme 2.8) et il suffit de voir que $M^{\perp_{b}}$ est inclus dans $M^{\perp}$. Dans le cas contraire, la relation

$$
\frac{1}{2}\left\langle X_{t}, X_{u}\right\rangle_{I}=X_{t}\{u\}=|\langle t, u\rangle|^{2}-|b(t, u)|^{2} \quad\left(t, u \in \boldsymbol{k}^{m}\right)
$$

montre qu'il existe un vecteur de la forme $X_{u}$ semi-positif, ce qui est absurde (lemme 1.1, 2).

Supposons de plus que $A$ est non dégénéré dans $V_{f, b}^{\rho}$. Soit $N$ un sousmodule de $M^{\perp}$. Alors $P_{N}$ commute avec $\varsigma$ ainsi que $\psi_{K}\left(P_{N}\right)$, autrement dit $\pi_{b}\left(P_{N}\right) \in T_{I} \Sigma_{b}^{\varsigma}$. Comme $M$ est stable par $\tau K, \pi_{b}\left(P_{N}\right)$ s'annule sur $M$, donc $\pi_{b}\left(P_{N}\right)=0$ et $N$ est stable par $\tau K$.

Remarque 2.13. - La condition suffisante de non dégénérescence 1) est valable même si $b$ n'est pas réflexive (on n'utilise pas le fait que $\Psi_{K}$ est involutive).

Pour avoir des informations plus fines on est amené à faire des hypothèses sur la représentation $\rho$. Nous dirons que $\rho: \Pi \rightarrow \mathrm{GL}_{m}(k)$ est diagonalisable si elle est somme directe de représentations de dimension 1 agissant par homothéties centrales. Dans ce cas $\rho$ se factorise par un groupe d'exposant 2 si $\boldsymbol{k}=\mathbb{R}$ ou $\mathbb{H}$ (conjuguer à une représentation unitaire) et par un groupe abélien si $\boldsymbol{k}=\mathbb{C}$. Inversement, il est clair que toute représentation dans $\mathrm{GL}_{m}(k)$ d'un groupe fini abélien $\Pi$, d'exposant 2 si $\boldsymbol{k}=\mathbb{R}$ ou $\mathbb{H}$, est diagonalisable. Les composants isotypiques des $\Pi$-modules $N$ sont du type «sous-espaces propres » :

$$
E_{\chi}=\{u \in N ; \pi \cdot u=u \chi(\pi), \forall \pi \in \Pi\},
$$

où $\chi$ est un caractère de $\Pi$ à valeurs dans le centre de $\boldsymbol{k}$. Bien qu'étant assez particulières, les représentations diagonalisables permettent de traiter des cas intéressants et significatifs, comme l'invariant de Bergé-Martinet (§ 2.10).

Remarque 2.14. - Si $\rho$ est diagonalisable, un sous-module $N$ est totalement stable par $\tau K$ si et seulement si $\tau K$ stabilise tout composant isotypique $E$ de $N$ en y induisant une homothétie centrale si $\operatorname{dim}_{k} E \geq 2$. En effet les composants isotypiques de $N$ sont ici de la forme (2.20) et toutes leurs droites sont des sous-modules.

Corollaire 2.16 (cas diagonalisable). - Soit $\rho$ comme dans le théorème 2 avec $\Pi$ abélien et supposé d'exposant 2 si $\boldsymbol{k}=\mathbb{R}$ ou $\mathbb{H}$, et soit $n_{\rho}$ le nombre de composants isotypiques de $\rho$. On note $a_{b}$ la dimension maximale d'un sousespace anisotrope, i.e. sans vecteurs isotropes, pour $b$.

Soit $A \in V_{f, b}^{\rho}$ eutactique et non dégénéré pour $\mu^{D}$ (par exemple parfait et eutactique). Alors les composants isotypiques de $M^{\perp}$ (resp. de $M_{A}^{\perp^{A}}$ ) sont anisotropes pour b (resp. pour $f$ ) et on a l'inégalité

$$
\operatorname{dim}_{\boldsymbol{k}} M^{\perp} \leq n_{\rho} a_{b} \leq|\Pi| a_{b} .
$$

BULLETIN DE LA SOCIÉTÉ MATHÉMATIQUE DE FRANCE 
En particulier, si b est symplectique $(\boldsymbol{k}=\mathbb{R}$ ou $\mathbb{C})$ les vecteurs minimaux de $A$ engendrent $\boldsymbol{k}^{m}$, et si b est symétrique sur $\mathbb{C}$ ou antihermitienne sur $\mathbb{H}$, on a $\operatorname{codim}_{\boldsymbol{k}} M \leq n_{\rho} \leq|\Pi|$.

Preuve. - Soit $u \in M^{\perp}$ dans un composant isotypique. D'après le théorème 2, 2) et la remarque 2.14, il existe $\alpha_{u} \in \boldsymbol{k}$ de module 1 tel que $\tau K u=u \alpha_{u}$, d'où $b(u, u)=|u|^{2} \alpha_{u}$. Les composants isotypiques de $M^{\perp}$ sont donc de dimension majorée par $a_{b}$ et leur nombre $n_{\rho}$ est au plus égal au nombre de caractères « centraux » de $\Pi$, c'est-à-dire $|\Pi|$. Dans le cas symplectique on a $a_{b}=0$. Si $b$ est symétrique sur $\mathbb{C}$ ou antihermitienne sur $\mathbb{H}$ il est facile de voir que tout plan possède des vecteurs isotropes, donc $a_{b}=1$.

EXEMPLE 2.15. - Il se peut effectivement que $M$ soit de grande codimension. Dans $\mathbb{R}^{p} \oplus \mathbb{R}^{q}(p=8 k, k \geq 1$ et $q \geq 1)$ prenons $K=I_{p, q}$ (forme de signature $(p, q)$ usuelle) et considérons le réseau orthogonal impair $\Lambda=E_{8}^{k} \oplus \mathbb{Z}^{q}$. Alors $\Lambda$ est eutactique car les gradients des vecteurs minimaux sont tous nuls, $M=\mathbb{R}^{q}$ et $\Lambda$ est non dégénéré (théorème 2,1 ). Noter que si $q=1$, il n'y a qu'un seul couple de vecteurs minimaux et $\Lambda$ est un minimum local strict de $\mu$ parmi les réseaux orthogonaux de même type (proposition 1.6, 2a).

Pour tout module semi-simple $M$, on note $\mathcal{T}_{M}$ l'ensemble des types qui apparaissent dans $M$ et $\bigoplus_{S \in \mathcal{T}_{M}} M_{S}$ la décomposition de $M$ en composants isotypiques.

THÉorÈme 3 (rang des vecteurs minimaux pour les points parfaits)

Soit $\rho: \Pi \rightarrow G \cap \mathrm{GL}_{m}(\mathcal{O})$ et soit $A \in V_{f, b}^{\rho}$ un point parfait pour $\mu^{D}$.

1) Posons $N=M^{\perp} \cap M^{\perp_{b}}$. Alors le sous-module $\bigoplus_{S \in \mathcal{T}_{N}} \boldsymbol{k}_{S}^{m}$ est totalement stable par $\tau K$.

2) Dans le cas hermitien complexe $(\boldsymbol{k}=\mathbb{C}, \tau=i d)$, symplectique réel ou antihermitien sur $\mathbb{H}(\boldsymbol{k}=\mathbb{R}$ ou $\mathbb{H}, \kappa=-1)$, et si $\rho$ est à valeurs dans $U_{f}^{*}(\mathcal{O})$, on a $N=M^{\perp}=M^{\perp_{b}}$.

3) Si $\rho$ est isotypique (par exemple triviale) et $V_{f, b}^{\rho}$ non réduite à un point, on a $N=\{0\}$, donc $\operatorname{dim}_{\boldsymbol{k}} M \geq \frac{1}{2} m$ et même $M=\boldsymbol{k}^{m}$ sous les hypothèses $d u 2)$.

Preuve. - 1) Posons $E=M^{\perp}, F=M^{\perp_{b}}$ et notons $T$ l'ensemble des vecteurs minimaux du réseau associé à $A$ par (2.17). La perfection de $A$ entraîne que tout vecteur tangent $X \in T_{I} \Sigma_{b}^{\varsigma}$ est combinaison $\mathbb{R}$-linéaire des $X_{t}^{\varsigma}$, donc des $X_{t}$ $(t \in T)$. Mais $X_{t}$ s'annule sur $N$ et on a $P_{E} X_{t} P_{F}=P_{F} X_{t} P_{E}=0$. Par suite

$$
X P_{N}=0 \quad \text { et } \quad P_{E} X P_{F}=P_{F} X P_{E}=0 \quad\left(X \in T_{I} \Sigma_{b}^{\varsigma}\right) .
$$

Soit $S$ un sous-module simple de $N$, et soit $L$ un sous-module de $\boldsymbol{k}^{m}$ isomorphe à $S$. Si $L$ n'est pas inclus dans $S^{\perp}$, l'application $P_{L} P_{S}$ induit un isomorphisme 
de $S$ sur $L$; on a $P_{L} P_{S}=P_{\tau K L} P_{S}$ d'après $(2.21)$, d'où $\tau K L=L$. Par continuité de $\tau K$, tout sous-module de $\boldsymbol{k}^{m}$ isomorphe à $S$ est stable par $\tau K$.

2) Dans les cas considérés, il existe $\delta \in \boldsymbol{k}$ central tel que $K^{2}=-\delta^{2} I$. On observe alors que la matrice $X_{Y}=\delta K^{*} Y+\delta^{*} Y K$ appartient à $T_{I} \Sigma_{b}^{\varsigma}$, pour tout $Y \in H_{m}(\boldsymbol{k}) \cap \mathcal{L}_{\varsigma}\left(\boldsymbol{k}^{m}\right)$ (on rappelle que $\varsigma$ est ici à valeurs dans $U_{b}(\boldsymbol{k})$ ). D'après (2.21), on a $P_{E} X_{Y} P_{W}=0$ où $W=M \cap M^{\perp_{b}}$. En prenant $Y=P_{W}$, et sachant que $K W$ est un sous-espace de $E=M^{\perp}$ disjoint de $W$, on voit que $W=\{0\}$. En utilisant encore $(2.21)$ avec $X=P_{E}-P_{F}$ on obtient que $P_{E}$ et $P_{F}$ commutent, de sorte que $F=W \oplus N$, ce qui achève la preuve de 2).

3) Si $N$ n'est pas nul, alors $\boldsymbol{k}^{m}$ est totalement stable par $\tau K$ d'après 1 ) et $V_{f, b}^{\rho}$ est réduite à un point (lemme $\left.2.15,2\right)$.

Corollaire 2.17 (cas diagonalisable). - Sous les hypothèses du corollaire 2.16 ( $\rho$ diagonalisable), on a

$$
\operatorname{dim}_{k} M \geq c\left(m-n_{\rho} a_{b}\right) \geq c\left(m-|\Pi| a_{b}\right) \quad \text { ò̀ } c=\frac{1}{2}
$$

pour tout point parfait pour $\mu^{D}$ dans $V_{f, b}^{\rho}$. Si de plus $f$ et $\rho$ vérifient les hypothèses de l'assertion 2) du théorème 3 , les inégalités (2.22) sont vraies avec $c=1$.

Preuve. - On majore la dimension du sous-module $M^{\perp} \cap M^{\perp_{b}}$ comme dans la preuve du corollaire 2.16 .

Corollaire 2.18. - Dans le cas symplectique réel ou complexe, supposons que tous les types de $\rho$ sur $\boldsymbol{k}$ sont de dimension impaire. Soit $A \in V_{f, b}^{\rho}$ un point eutactique non dégénéré ou parfait pour $\mu^{D}$ (on suppose de plus $\rho$ symplectique si A est parfait). Alors les vecteurs minimaux de A engendrent $\boldsymbol{k}^{m}$.

Preuve. - Tout sous-espace de $\boldsymbol{k}^{m}$ invariant par l'application semi-linéaire $\tau K$ $\left(\tau=\sigma\right.$ si $\boldsymbol{k}=\mathbb{C}$ ) est de dimension paire. On a donc $M^{\perp}=\{0\}$ (théorème 2, 2) et théorème 3,1$), 2)$ ).

REMARQue 2.16. - Tous les énoncés précédents sont indépendants du théorème de Voronoï, qui d'ailleurs n'est pas toujours vérifié, même dans les meilleurs cas (voir remarque 2.7).

2.9. Finitude des classes minimales pour les réseaux autoduaux. Soit $b$ comme au $\S 2.7$ et soit $V_{f, b}$ l'espace des réseaux $b$-autoduaux marqués de type $f$ (définition 2.2). On sait par un argument topologique que les classes minimales non isotropes de $V_{f, b}$ sont en nombre fini modulo $\mathrm{SU}_{f}^{*}(\mathcal{O})$ (proposition $2.13,2$ ). Dans le cas réflexif, et pour certains réseaux autoduaux, une étude algébrique des classes isotropes permet de compléter ce résultat. 
ThÉORÈme 4. - On suppose que le corps de base est $\boldsymbol{k}=\mathbb{R}$ ou $\mathbb{C}$, et que b est soit hermitienne, soit bilinéaire symétrique ou antisymétrique. Alors les classes minimales de $V_{f, b}$ pour $\mu$ sont en nombre fini modulo l'action de $\mathrm{SU}_{f}^{*}(\mathcal{O})$.

Lemme 2.19. - Soit $\mathcal{O}=\mathbb{Z}$ ou $\mathbb{Z}[i]$, et soit $\left(\mathcal{O}^{m}, f\right)$ un $\mathcal{O}$-module bilinéaire symétrique, bilinéaire antisymétrique ou hermitien que l'on suppose régulier (i.e. le déterminant de $f$ est inversible). Pour tout sous-module $V$ de $\mathcal{O}^{m}$, on note $N_{V}$ le cardinal du sous-groupe de torsion de $\mathcal{O}^{m} / V$.

1) Soit $V$ un sous-module totalement isotrope. Alors le sous-groupe des éléments de $\mathrm{GL}(V)$ qui se prolongent en un élément de $\mathrm{SU}_{f}(\mathcal{O})$ est d'indice fini (que l'on peut borner en fonction de $m$ et $N_{V}$ ).

2) Soit $N \geq 1$. Modulo l'action de $\mathrm{SU}_{f}(\mathcal{O})$, il n'existe qu'un nombre fini de sous-modules totalement isotropes $V$ avec $N_{V} \leq N$.

Preuve du théorème 4. - D'après la proposition 2.13, 2), il reste à montrer que les configurations $S$ de vecteurs minimaux (pour les formes de $V_{f, b}$ ) qui engendrent un sous-module $V(S)$ totalement isotrope de $\left(\mathcal{O}^{m}, f\right)$ sont en nombre fini modulo $\mathrm{SU}_{f}(\mathcal{O})$. Comme les configurations de vecteurs minimaux des réseaux euclidiens ou hermitiens sont en nombre fini modulo $\mathrm{GL}\left(\mathcal{O}^{m}\right)$ (voir [11, prop. 3.2] pour le cas réel et corollaire 2.6, 2), le cardinal de la torsion $N_{V}$ est majoré par un entier ne dépendant que de $m$. Par le lemme 2.19, 2), les $V(S)$ sont donc $\mathrm{SU}_{f}(\mathcal{O})$-équivalents à un nombre fini de sous-modules totalement isotropes. Soit $V=V(S)$ fixé. Les configurations minimales des réseaux euclidiens ou hermitiens de $V \otimes_{\mathcal{O}} \boldsymbol{k}(\boldsymbol{k}=\mathbb{R}$ ou $\mathbb{C})$ sont en nombre fini modulo $\mathrm{GL}(V)$ (loc. cit.). Mais d'après le 1 ) du lemme 2.19 , les configurations $\mathrm{GL}(V)$-équivalentes se répartissent en un nombre fini de classes sous l'action de $\operatorname{SU}_{f}(\mathcal{O})$, d'où la finitude.

Preuve du lemme 2.19. - 1) Pour tout entier $N \geq 1$, on note $\Gamma_{V}(N)$ le noyau du morphisme naturel de GL $(V)$ dans GL $(V / N V)$. Par le théorème de la base adaptée, on voit que $V$ est inclus dans un sous-module $W$ isotrope, facteur direct et de même rang que $V$. On vérifie alors (en prenant une base adaptée) que tout élément de $\Gamma_{V}\left(N_{V}\right)$ se prolonge en un élément de $\mathrm{GL}(W)$. On peut donc supposer que $V$ est facteur direct dans $\mathcal{O}^{m}$.

Affirmation. - Soit $k$ le rang de $V$. Il existe un sous-ensemble fini $\mathcal{M}$ de matrices diagonales $k \times k$ à coefficients dans $\mathcal{O}, \mathcal{M}$ indépendant de $V$, et il existe un sous-module $F$ de rang $2 k$ contenant $V$ et muni d'une base $\mathcal{B}$ dans laquelle la matrice de $f$ est de la forme $\left(\begin{array}{cc}0 & I \\ \epsilon I & D\end{array}\right)$, avec $D \in \mathcal{M}$ et $\epsilon=-1$ si $f$ est symplectique, $\epsilon=1$ sinon.

On prouve cet énoncé par récurrence sur $k$. En effet il existe $v \in V$ indivisible ( $V$ est facteur direct) et $w \in \mathcal{O}^{m}$ tel que $f(v, w)=1$. Posons $f(w, w)=2 \lambda+r$ (division euclidienne). Alors $f(w-v \lambda, w-v \lambda)=r$ (par exemple on peut choisir $f(w, w)=0$ si $f$ est hermitienne paire). Soit $P$ le plan engendré par $v$ 
et $w$. On a $V=V \cap P \oplus V \cap P^{\perp}$ : si $x \in V$, alors $x-v f(x, w)$ (cas bilinéaire) ou $x-v f(x, w)^{\sigma}$ (cas hermitien) appartient à $V \cap P^{\perp}$.

D'après l'affirmation, on a $\mathcal{O}^{m}=F \oplus F^{\perp}$ et il suffit de prolonger matriciellement (en utilisant la base $\mathcal{B}$ ) les éléments de $\mathrm{GL}(V)$ en isométries de $F$. La matrice $\left(\begin{array}{cc}A & 0 \\ C & \left(A^{-1}\right)^{* \tau}\end{array}\right) \in M_{2 k}(\mathcal{O})$ définit une isométrie de $F$ si et seulement si

$$
\epsilon C A^{* \tau}+A C^{* \tau}+D=A D A^{* \tau}
$$

(on rappelle que $\tau$ est la conjugaison dans le cas bilinéaire complexe, l'identité sinon). Si $\epsilon=-1$ ( $f$ symplectique) on a $D=0$ et on prend $C=0$. Si $\epsilon=1$ et si $A$ représente un élément de $\Gamma_{V}(2) \cap \mathrm{SL}(V)$, alors $C=\frac{1}{2}\left[A D-D\left(A^{-1}\right)^{* \tau}\right]$ convient (noter que $D^{* \tau}=D$ ).

2) Il revient au même de prouver la finitude pour l'action de $\mathrm{U}_{f}(\mathcal{O})$. Comme chaque sous-module contient un nombre fini de sous-modules d'indice au plus $N$, on peut se restreindre aux facteurs directs (de rang fixé). Deux facteurs directs totalement isotropes de même rang $V_{j}(j=1,2)$ auxquels on associe des sous-modules $F_{j}$ et des matrices $D_{j} \in \mathcal{M}$ (affirmation) seront $U_{f}(\mathcal{O})$-équivalents dès que $D_{1}=D_{2}$ et $\left(F_{1}^{\perp}, f\right)$ isométrique à $\left(F_{2}^{\perp}, f\right)$. C'est donc le cas si $f$ est symplectique $\left(D_{1}=D_{2}=0\right)$. Plus généralement, si $p$ et $\delta$ sont fixés, il n'existe qu'un nombre fini (à isomorphisme près) de $\mathcal{O}$-modules bilinéaires symétriques ou hermitiens $\left(\mathcal{O}^{p}, g\right)$ avec $0<|\operatorname{det} g| \leq \delta$, en particulier réguliers. C'est bien connu pour le cas réel défini positif, et la même preuve (par récurrence sur $p$ ) convient dans les autres cas, pourvu que l'on puisse trouver $w \in \mathcal{O}^{p}$ avec $0<|g(w, w)| \leq C_{p, \delta}$, où $C_{p, \delta}$ ne dépend que de $p$ et de $\delta$ (voir par exemple [29, p. 18]). En réduisant la forme $g$ sur $\boldsymbol{k}^{p}$, on trouve $P \in \mathrm{GL}_{p}(\boldsymbol{k})$ avec $0<|\operatorname{det} P| \leq \delta^{1 / 2}$ et $K \in \mathrm{U}_{p}(\boldsymbol{k})$ tels que $g(x, y)=\left\langle(P x)^{\tau *}, K P y\right\rangle$ (par exemple $K=I$ pour le cas symétrique complexe). Mais il existe $v \in \mathcal{O}^{p}$ tel que $|P v|^{2} \leq C_{p} \delta^{1 / p}$ avec $C_{p}=\gamma_{p}$ ou $\gamma_{2 p}$ (constantes d'Hermite) suivant que $\boldsymbol{k}=\mathbb{R}$ ou $\boldsymbol{k}=\mathbb{C}$. Par suite $|g(v, v)| \leq|P v|^{2}$ est petit. Si $v$ est isotrope et indivisible, on peut choisir $w \in \mathcal{O}^{p}$ tel que $g(v, w)=1$ et $0<|g(w, w)| \leq 2$ en utilisant la division euclidienne par 2 (voir la preuve de l'affirmation). Ce qui achève la preuve du lemme.

\subsection{Interprétation symplectique de l'invariant de Bergé-Martinet}

Rappelons (voir [8], [28, ch. II, § 8]) que l'invariant de Bergé-Martinet d'un réseau réel est défini par

$$
\gamma_{m, k}^{\prime \mathbb{R}}(\Lambda)=\left[\delta_{m, k}^{\mathbb{R}}(\Lambda) \delta_{m, k}^{\mathbb{R}}\left(\Lambda^{*}\right)\right]^{1 / 2} \quad(1 \leq k \leq m),
$$

où $\delta_{m, k}(\Lambda)$ est la borne inférieure des déterminants de Gram des sous-réseaux de dimension $k$ de $\Lambda$. La quantité normalisée $\gamma_{m, k}^{\mathbb{R}}=\delta_{m, k}^{\mathbb{R}} /\left(\delta_{m, m}^{\mathbb{R}}\right)^{k / m}$ est l'invariant de Rankin [32] ou « $k$-invariant d'Hermite ». Ces notions ont aussi un sens pour les réseaux hermitiens complexes ou quaternioniens et on a de même des 
invariants $\gamma_{m, k}^{\boldsymbol{k}}$ et $\gamma_{m, k}^{\prime \boldsymbol{k}}(\boldsymbol{k}=\mathbb{C}$ ou $\mathbb{H})$. Comme on ne considère que des sousréseaux de même nature que $\Lambda$, il y a des relations évidentes quand on passe a un sous-corps, par exemple pour $\Lambda$ complexe on a $\left[\delta_{m, k}^{\mathbb{C}}(\Lambda)\right]^{2} \geq \delta_{2 m, 2 k}^{\mathbb{R}}\left(\Lambda_{\mathbb{R}}\right)$.

Commençons par donner une description symplectique de l'invariant de Bergé-Martinet classique $\gamma^{\prime}=\gamma_{m, 1}^{\prime \mathbb{R}}$. Bien qu'évidente, elle permettra d'appliquer les résultats du $\S 2.8$. On note $H_{m}^{+}(\boldsymbol{k})$ le cône des matrices hermitiennes définies positives $(\boldsymbol{k}=\mathbb{R}, \mathbb{C}$ ou $\mathbb{H}$ ). Les réseaux symplectiques (réels) sont les réseaux autoduaux pour une forme bilinéaire symplectique, qui peut toujours être ramenée à $\omega(x, y)=x^{t} J_{m} y,\left(x, y \in \mathbb{R}^{m}\right)$ avec $J_{m}$ définie par (2.13). Il n'y a qu'un seul type $F=J_{m}$. L'espace des réseaux symplectiques marqués est donc (proposition 2.9, 2)

$$
\mathfrak{S}_{m}=\left\{A \in P_{2 m}^{\mathbb{R}} ; A J_{m} A=J_{m}\right\} .
$$

Soit $\rho$ la représentation de $\mathbb{Z} / 2 \mathbb{Z}$ dans $\operatorname{MSp}_{2 m}(\mathbb{Z})$ définie par $\rho(1)=\left(\begin{array}{ll}I_{m} & 0 \\ 0 & -I_{m}\end{array}\right)$ qui change le signe de $\omega$. Le lieu invariant $\mathfrak{S}_{m}^{\rho}$ est paramétré par le cône $H_{m}^{+}(\mathbb{R})$ :

$$
\mathfrak{S}_{m}^{\rho}=\left\{\tau_{A} ; A \in H_{m}^{+}(\mathbb{R})\right\}, \quad \text { où } \quad \tau_{A}=\left(\begin{array}{cc}
A & 0 \\
0 & A^{-1}
\end{array}\right) .
$$

Naturellement, le groupe des matrices $\left(\begin{array}{l}P \\ 0 \\ 0\left(P^{t}\right)^{-1}\end{array}\right)$ où $P \in \mathrm{GL}_{2 m}(\mathbb{R})$ est d'indice fini dans le commutant $\operatorname{MSp}_{2 m}^{\rho}(\mathbb{R})$. L'étude de $\gamma^{\prime}$ est ramenée à celle de $\mu$ sur $\mathfrak{S}_{m}^{\rho}$. En effet on a

$$
\mu\left(\tau_{A}\right)=\min \left(\delta_{m, 1}^{\mathbb{R}}(A), \delta_{m, 1}^{\mathbb{R}}\left(A^{-1}\right)\right) \leq \gamma^{\prime}(A)
$$

pour tout $A \in H_{m}^{+}(\mathbb{R})$, avec égalité si et seulement si $A$ appartient à l'ensemble $\Delta_{m, 1}^{\mathbb{R}}$ des points tels que $\delta_{m, 1}^{\mathbb{R}}(A)=\delta_{m, 1}^{\mathbb{R}}\left(A^{-1}\right)$. Comme les deux facteurs de $\mathbb{R}^{2 m}=\mathbb{R}^{m} \oplus \mathbb{R}^{m}$ sont totalement isotropes pour $\omega$, tout point de $\mathfrak{S}_{m}^{\rho}$ faiblement eutactique (par exemple extrême ou parfait) pour $\mu$ est de la forme $\tau_{A}$ avec $A \in \Delta_{m, 1}^{\mathbb{R}}$ (théorème 1,1 ). Par ailleurs, il y a clairement bijection entre les points $\tau_{A}$ extrêmes pour $\mu$ dans $\mathfrak{S}_{m}^{\rho}$ et les points $A \in \Delta_{m, 1}^{\mathbb{R}}$ extrêmes pour $\gamma^{\prime}$.

Proposition 2.20. - Un point $\tau_{A}\left(A \in \Delta_{m, 1}^{\mathbb{R}}\right)$ est parfait pour $\mu$ dans $\mathfrak{S}_{m}^{\rho}$ si et seulement si le couple $\left(A, A^{-1}\right)$ est parfait au sens de [28, ch. IX, déf. 6.2] (voir aussi $[7]$ ).

Preuve. - On se ramène au point $I=I_{m}$ en posant $A=P P^{t}\left(P \in \mathrm{GL}_{m}(\mathbb{R})\right)$. Soit $T$ (resp. $T^{*}$ ) l'ensemble des vecteurs minimaux de $\Lambda=P^{t} \mathbb{Z}^{m}\left(\operatorname{resp} . \operatorname{de} \Lambda^{*}\right)$. La perfection de $\tau_{A}\left(A \in \Delta_{m, 1}^{\mathbb{R}}\right)$ s'exprime avec les gradients des fonctions longueur dans l'espace des paramètres $H_{m}^{+}(\mathbb{R})$ (au point $I$, pour la métrique $\langle., .\rangle_{I}$ ) par le fait que $\left(u u^{t}\right)_{u \in T} \cup\left(-v v^{t}\right)_{v \in T^{*}}$ engendre affinement $H_{m}(\mathbb{R})$. Le couple $\left(A, A^{-1}\right)$ est parfait si pour tout $\left(u_{0}, v_{0}\right) \in T \times T^{*}$, le rang (vectoriel) de la famille $\left(u u^{t}-u_{0} u_{0}^{t}\right)_{u \in T} \cup\left(v v^{t}-v_{0} v_{0}^{t}\right)_{v \in T^{*}}$ vaut $\frac{1}{2} m(m+1)-1$. En observant 
que les familles de points $\left(u u^{t}\right)_{u \in T}$ et $\left(-v v^{t}\right)_{v \in T^{*}}$ sont situées dans deux hyperplans affines parallèles et distincts (ici orthogonaux à $I$ ), l'équivalence des deux conditions est un exercice élémentaire.

REMARQUe 2.17. - La construction analogue avec les réseaux symplectiques complexes n'est pas reliée à $\gamma_{m, 1}^{\prime \mathbb{C}}$, mais à $\delta_{m, 1}^{\mathbb{C}}(\Lambda) \delta_{m, 1}^{\mathbb{C}}\left(\Lambda^{* \sigma}\right)$.

Note 2.18. - Soit $\mathcal{F}=\left(u u^{t}\right)_{u \in T} \cup\left(-v v^{t}\right)_{v \in T^{*}}$ comme dans la preuve de la proposition 2.20. Le réseau $\Lambda$ est « dual-parfait » (voir [8]) si $\mathcal{F}$ engendre vectoriellement $H_{m}(\mathbb{R})$, tandis que (proposition 2.20) le couple $\left(\Lambda, \Lambda^{*}\right)$ est parfait si $\mathcal{F}$ engendre affinement $H_{m}(\mathbb{R})$. La « duale-eutaxie » (voir [8]) correspond bien au fait que $\mathcal{F}$ est eutactique (définition 1.1). Évidemment dans ce cas, et même pour $\mathcal{F}$ faiblement eutactique, rang affine et rang vectoriel coïncident, donc $\Lambda$ dual-parfait équivaut à $\left(\Lambda, \Lambda^{*}\right)$ parfait. Le théorème de Voronoï pour $\gamma^{\prime}$, établi dans [8], peut donc être énoncé avec l'une ou l'autre notion (voir [7]). D'après la proposition 1.8, tout couple parfait est « quasi-parfait » (voir [28, ch. IX, déf. 6.4]).

On obtient (théorème $1,2 \mathrm{~b}$ ) et corollaire $2.12,2$ ) la finitude et l'algébricité des couples parfaits [7, théorème 3.3], mais aussi celles des réseaux dualeutactiques non dégénérés (théorème $1,2 \mathrm{c}$ ). Pour ces réseaux, on voit que $T$ et $T^{*}$ engendrent $\mathbb{R}^{2 m}$ (corollaire 2.16 ), propriété connue pour les réseaux dualextrêmes (voir [28, ch. III, prop. 8.2]).

Les considérations précédentes indiquent une approche naturelle pour l'étude de $\gamma_{m, k}^{\prime k}$ pour $\boldsymbol{k}=\mathbb{R}$ ou $\mathbb{C}$ et $1 \leq k \leq m$. Posons

$$
\nu_{m, k}^{k}(A)=\min \left(\delta_{m, k}^{\boldsymbol{k}}(A), \delta_{m, k}^{\boldsymbol{k}}\left(A^{-1}\right)\right) \quad\left(A \in H_{m}^{+}(\boldsymbol{k})\right),
$$

avec $\delta_{m, k}^{\boldsymbol{k}}(A)=\delta_{m, k}^{\boldsymbol{k}}\left(P^{*} \mathcal{O}^{m}\right)$ si $A=P P^{*}\left(P \in \mathrm{GL}_{m}(\boldsymbol{k})\right)$. Soit $M_{m, k}^{\times}(\mathcal{O})$ l'ensemble des matrices de rang $k$ de $M_{m, k}(\mathcal{O})$. Comme pour $\boldsymbol{k}=\mathbb{R}$ (voir [5, $\S 2.6]), \delta_{m, k}^{k}$ est associé au système de fonctions longueur définies $\operatorname{sur} H_{m}^{+}(\boldsymbol{k})$ par $f_{U}(A)=\operatorname{det}\left(U^{*} A U\right.$ ) et paramétrées par $U \in M_{m, k}^{\times}(\mathcal{O})$ (ou plutôt si $U=\left(u_{1}, \ldots, u_{k}\right)$ par $u_{1} \wedge \cdots \wedge u_{k} \neq 0 \in \wedge^{k}\left(\mathcal{O}^{m}\right)$ pour avoir la finitude locale des « petites longueurs»). Posons $\ell_{U, V}(A)=f_{U}(A)+f_{V}\left(A^{-1}\right)$ pour $A \in H_{m}^{+}(\boldsymbol{k})$ et $U, V \in M_{m, k}^{\times}(\mathcal{O})$, et

$$
\mathcal{C}_{m, k}=\left\{(U, V) \in\left(M_{m, k}^{\times}(\mathcal{O})\right)^{2} ; U=0 \text { ou } V=0\right\},
$$

ensemble stable par l'action à droite de $\operatorname{GL}_{m}(\mathcal{O}) \operatorname{sur}\left(M_{m, k}^{\times}(\mathcal{O})\right)^{2}$, i.e. $(U, V) \cdot P=\left(P^{*} U, P^{-1} V\right)$. Alors $\nu_{m, k}^{k}$ est défini par le système de fonctions longueur $\left(\ell_{U, V}\right)_{(U, V) \in \mathcal{C}_{m, k}}$.

Proposition 2.21. - Soit $\Delta_{m, k}^{\boldsymbol{k}}=\left\{A \in H_{m}^{+}(\boldsymbol{k}) ; \delta_{m, k}^{\boldsymbol{k}}(A)=\delta_{m, k}^{\boldsymbol{k}}\left(A^{-1}\right)\right\}$.

1) Tout point $A$ faiblement eutactique pour $\nu_{m, k}^{k}$ appartient $\grave{a} \Delta_{m, k}^{k}$. 
2) Il y a bijection entre les points extrêmes (resp. strictement extrêmes) pour $\nu_{m, k}^{k}$ et les points $A \in \Delta_{m, k}^{k}$ extrêmes (resp. strictement extrêmes dans $\left.\Delta_{m, k}^{k}\right)$ pour $\gamma_{m, k}^{\prime k}$.

Preuve. - 1) Soit $U \in M_{m, k}^{\times}(\boldsymbol{k})$ et soit $P_{U}=U\left(U^{*} U\right)^{-1} U^{*}$ la matrice de la projection orthogonale sur le sous-espace de $\boldsymbol{k}^{m}$ engendré par les colonnes $u_{1}, \ldots, u_{k}$ de $U\left(P_{U}\right.$ ne dépend que de $\left.u_{1} \wedge \cdots \wedge u_{k}\right)$. Le gradient de $f_{U}$ au point $I$ pour $\langle., .\rangle_{I}$ est donné par

$$
\nabla f_{U}(I)=\operatorname{det}\left(U^{*} U\right) P_{U} .
$$

On prouve alors l'assertion par l'absurde à partir du lemme 1.1,4) en effectuant une variation au point $I$ dans la direction de $I$ (ce qui correspond à une variation radiale au point $A$ ).

2) On a $\nu_{m, k}^{k}(A) \leq \gamma_{m, k}^{\prime k}(A)$ avec égalité si et seulement si $A \in \Delta_{m, k}^{k}$. Sachant que $\gamma_{m, k}^{\prime k}$ est déterminé par ses valeurs sur $\Delta_{m, k}^{k}$ et que les points extrêmes de $\nu_{m, k}^{k}$ sont situés sur $\Delta_{m, k}^{k}$ d'après 1 ), on en déduit facilement le résultat.

Par conséquent, l'étude des points $\gamma_{m, k}^{\prime k}$-extrêmes équivaut à celle des points $\nu_{m, k}^{k}$-extrêmes. On conviendra de dire qu'un réseau $\Lambda$ avec $\delta_{m, k}^{k}(\Lambda)=\delta_{m, k}^{k}\left(\Lambda^{*}\right)$ est parfait (resp. eutactique, semi-eutactique, faiblement eutactique) pour $\gamma_{m, k}^{\prime k}$ s'il l'est pour $\nu_{m, k}^{k}$. Ces notions s'explicitent avec la définition 1.1 appliquée à la famille $\left(P_{U}\right)_{U \in T} \cup\left(-P_{V}\right)_{V \in T^{*}}$ du $\mathbb{R}$-espace vectoriel $H_{m}(\boldsymbol{k})$, où $T$ (resp. $T^{*}$ ) représente l'ensemble des sous-réseaux de déterminant minimal de $\Lambda$ (resp. de $\Lambda^{*}$ ). Le résultat suivant répond au problème 6.10 de [28], p. 310, posé pour $\boldsymbol{k}=\mathbb{R}$.

ThÉORÈme 5 (condition $(C)$ pour $\left.\nu_{m, k}^{\boldsymbol{k}}\right)$. - L'invariant $\nu_{m, k}^{\boldsymbol{k}}(\boldsymbol{k}=\mathbb{R}$ ou $\mathbb{C}$, $0 \leq k \leq m)$ satisfait la condition $(C)$ de $[5, \S 2.2]$, donc le théorème de Voronoï. Par conséquent, l'invariant $\gamma_{m, k}^{\prime k}$ défini sur le projectifié du cône $H_{m}^{+}(\boldsymbol{k})$ vérifie le théorème de Voronoï (avec les définitions ci-dessus).

Preuve. - On se ramène au point $I$ par l'action de $\mathrm{GL}_{m}(\boldsymbol{k})$. Soit a un réel positif et soit $T$ un sous-ensemble fini de $M_{m, k}^{\times}(\boldsymbol{k})$ tel que $\operatorname{det}\left(U^{*} U\right)=a$ pour tout $U \in T$. Soient $T^{+}$et $T^{-}$deux parties disjointes de $T$ telles que $T=T^{+} \cup T^{-}$; on pose $U^{+}=(U, 0)$ pour $U \in T^{+}$et $U^{-}=(0, U)$ pour $U \in T^{-}$. Soit $X \in H_{m}(\boldsymbol{k})$ orthogonal à $P_{U}\left(\right.$ i.e. $\left.\operatorname{Tr}\left(X P_{U}\right)=0\right)$ pour tout $U \in T$. Considérons enfin la courbe $c(t)=\exp \left(t X+\frac{1}{2} t^{2} Y\right)$ avec $Y \in H_{m}(\boldsymbol{k})$ à déterminer et posons $a h_{U^{\epsilon}}(t)=\ell_{U^{\epsilon}} \circ c(t)=f_{U} \circ \exp \left[\epsilon\left(t X+\frac{1}{2} t^{2} Y\right)\right](\epsilon= \pm)$. En développant $\operatorname{det}(I+M)$ suivant les fonctions symétriques des valeurs propres de $M$ (hermitienne) et en exprimant celles-ci avec les traces des puissances de $M$, on calcule les dérivées successives de $h_{U^{\epsilon}}$ en $t=0$ :

$$
h_{U^{\epsilon}}^{\prime}(0)=0, \quad h_{U^{\epsilon}}^{\prime \prime}(0)=\epsilon \operatorname{Tr}\left(P_{U} Y\right)+\operatorname{Tr}\left(P_{U} X^{2}\right)-\operatorname{Tr}\left(P_{U} X\right)^{2} .
$$


Pour toute matrice hermitienne $Z$, on a l'inégalité

$$
\operatorname{Tr}\left(P_{U} Z\right)^{2} \leq \operatorname{Tr}\left(P_{U} Z^{2}\right)
$$

avec égalité si et seulement si $Z$ commute avec $P_{U}$. Notons $T_{0}$ l'ensemble des $U \in T$ tels que $X P_{U}=P_{U} X$. Alors pour $U \in T_{0}$ on a

$$
h_{U^{\epsilon}}^{(3)}(0)=0, \quad h_{U^{\epsilon}}^{(4)}(0)=3\left[\operatorname{Tr}\left(P_{U} Y^{2}\right)-\operatorname{Tr}\left(P_{U} Y\right)^{2}+\left(\operatorname{Tr} P_{U} Y\right)^{2}\right] .
$$

Soit $E$ le sous-espace des $Y \in H_{m}(\boldsymbol{k})$ tel que $\operatorname{Tr}\left(P_{U} Y\right)=0$ pour tout $U \in T_{0}$. Les matrices $Y \in E$ qui commutent à $P_{U}$ ( $U$ fixé dans $T_{0}$ ) forment un sousespace strict de $E$ (voir la preuve de la proposition 2.8 de [5]). Par suite il existe $Y \in E$ tel que $Y P_{U} \neq P_{U} Y$ pour tout $U \in T_{0}$, de sorte que si $Y$ est assez petit, on a $h_{U^{\epsilon}}^{\prime \prime}(0)>0$ pour $U \in T \backslash T_{0}, h_{U^{\epsilon}}^{\prime \prime}(0)=h_{U^{\epsilon}}^{(3)}(0)=0$ et $h_{U^{\epsilon}}^{(4)}(0)>0$ pour $U \in T_{0}$, ce qui prouve la condition $(\mathrm{C})$.

2.11. Invariant d'Hermite-Humbert. — L'étude de l'invariant d'HermiteHumbert $\mu_{L}$ associé à un corps de nombre a été abordée dans [25, 3, 19] avec les méthodes traditionnelles de la théorie des réseaux. Nous analysons ici $\mu_{L}$ dans le cadre des systoles généralisées et nous donnons quelques propriétés additionnelles de cet invariant (condition $(C)$, finitude des points eutactiques non dégénérés).

Soit $L$ un corps de nombre de degré $m$ et soit $\mathcal{O}_{L}$ l'anneau de ses entiers. Le plongement canonique $\sigma$ de $L$ dans $\mathbb{R}^{r} \times \mathbb{C}^{s}(r+2 s=m)$ sera noté $\sigma(x)=$ $\left(x^{(k)}\right)_{1 \leq k \leq r+s}(x \in L)$. Pour $n \geq 1$, considérons l'espace des formes de Humbert

$$
P_{n, L}=\left(P_{n}^{\mathbb{R}}\right)^{r} \times\left(P_{n}^{\mathbb{C}}\right)^{s}
$$

(cette notation est légèrement différente de celle de $[3,19])$ et posons

$$
\mu_{L}(A)=\inf _{u \in \mathcal{O}_{L}^{n} \backslash\{0\}} \prod_{k=1}^{r+s} A_{k}\left\{u^{(k)}\right\}^{d_{k}}, \quad A=\left(A_{1}, \ldots, A_{r+s}\right) \in P_{n, L},
$$

avec $d_{k}=1$ (resp. $\left.d_{k}=2\right)$ si $k \leq r$ (resp. $\left.k>r\right)$. Il est clair que $\mu_{L}$ est invariant par $\mathrm{GL}_{n}\left(\mathcal{O}_{L}\right)$ agissant diagonalement sur $P_{n, L}$ via les plongements de $L$ dans $\mathbb{C}$. Soient $A \in P_{n, L}$ et $\lambda>0$ fixés. Modulo multiplication par les unités de $\mathcal{O}_{L}$, il n'existe qu'un nombre fini de vecteurs $u \in \mathcal{O}_{L}^{n}$ tels que $f_{u}(A)=\prod_{k=1}^{r+s} A_{k}\left\{u^{(k)}\right\}^{d_{k}} \leq \lambda$ (voir [25, p. 17], preuve du lemme 1). Comme de plus les gradients $(2.24)$ de $\log f_{u}$ sur $P_{n, L}$ ont une norme constante, on en déduit que $\mu_{L}$ est une systole généralisée (voir remarque 1.1).

Proposition 2.22 (condition $(C)$ pour l'invariant d'Hermite-Humbert)

1) Les notions de perfection et d'eutaxie pour les formes de Humbert introduites dans [19] coïncident avec perfection et eutaxie pour $\mu_{L}$ au sens de la définition 1.2 .

2) L'invariant $\mu_{L}$ vérifie la condition $(C)$. En particulier, on a un théorème de Voronö̈ pour $\mu_{L}$ (voir [19, théorème 3.5]).

BULlETIN DE LA SOCIÉTÉ MATHÉMATIQUE DE FRANCE 
Preuve. - 1) On peut remplacer $\mu_{L}$ par $\log \mu_{L}$ (remarque 1.2,2) qui est défini par des fonctions de la forme

$$
\ell_{U}(A)=\sum_{1 \leq k \leq r+s} d_{k} \log A_{k}\left\{u_{k}\right\}
$$

où $A=\left(A_{k}\right)_{k} \in P_{n, L}$ et $U=\left(u_{k}\right)_{k} \in \mathbb{R}^{r} \times \mathbb{C}^{s} \backslash \Delta$ avec $\Delta$ défini par $\prod_{k}\left|u_{k}\right|=0$. D'après (2.10), le gradient de $\ell_{U}$ pour la métrique riemannienne produit sur $P_{n, L}$ est donné par

$$
\nabla \ell_{U}(A)=\left(\frac{d_{k}}{A_{k}\left\{u_{k}\right\}} A_{k} u_{k} u_{k}^{*} A_{k}-\frac{d_{k}}{n} A_{k}\right)_{1 \leq k \leq r+s} .
$$

Noter que $\left\|\nabla \ell_{U}(A)\right\|^{2}=(1-1 / n)(r+4 s)$. Soit $S_{A}$ un système fini de représentants des vecteurs minimaux de $A$ pour $\mu_{L}$. Alors $A$ est eutactique s'il existe des réels $\rho_{u}>0\left(u \in S_{A}\right)$ tels que $\sum_{u \in S_{A}} \rho_{u} \nabla \ell_{\sigma(u)}(A)=0$, i.e.

$$
A_{k}^{-1}=n \sum_{u \in S_{A}} \frac{\rho_{u} u^{(k)} u^{(k) *}}{A_{k}\left\{u^{(k)}\right\}}
$$

pour tout $k=1, \ldots, r+s$ (définition 2.3 de [19]).

L'action diagonale du groupe $G^{r, s}=\mathrm{SL}_{n}(\mathbb{R})^{r} \times \mathrm{SL}_{n}(\mathbb{C})^{s}$ sur $P_{n, L}$ permet d'écrire $A=P \cdot I$ avec $P=\left(P_{k}\right)_{k} \in G^{r, s}$ et $I=\left(I_{n}\right)_{k}$. Alors $A$ est parfaite si et seulement si le rang affine de $\left(P^{-1} \cdot \nabla \ell_{\sigma(u)}(A)\right)_{u \in S_{A}}$ est maximal dans $T_{I} P_{n, L}$. Cela revient à dire que

$$
\left(\left(d_{k} P_{k}^{*} u^{(k)} u^{(k) *} P_{k} / A_{k}\left\{u^{(k)}\right\}\right)_{k}\right)_{u \in S_{A}}
$$

engendre vectoriellement $T_{I} P_{n, L} \oplus \mathbb{R}\left(d_{k} I_{n}\right)_{k}$ (voir [5, p. 106], preuve de la proposition 2.5), ou encore que le rang vectoriel de $\left(\left(u^{(k)} u^{(k) *}\right)_{k}\right)_{u \in S_{A}}$ vaut $\operatorname{dim}_{\mathbb{R}} P_{n, L}+1$ (définition 2.2,3) de [19]).

2) Comme $G^{r, s}$ est transitif sur $P_{n, L}$, il suffit d'établir la condition $(C)$ au point $A=I$ pour les longueurs $\ell_{U}$ avec $U \in \mathbb{R}^{r} \times \mathbb{C}^{s} \backslash \Delta$. Soient $\alpha \in \mathbb{R}$, $X \in T_{I} P_{n, L}$ non nul et $T$ un sous-ensemble fini de $\mathbb{R}^{r} \times \mathbb{C}^{s} \backslash \Delta$ tel que $\ell_{U}(I)=\alpha$ et $\mathrm{d} \ell_{U}(I) \cdot X=0$ pour tout $U \in T$. Posons $f_{U}(t)=\ell_{U} \circ \exp \left(t X+\frac{1}{2} t^{2} Y\right)$ où $Y \in T_{I} P_{n, L}$ est à déterminer. Pour $U \in T$ on a

$$
f_{U}^{\prime}(0)=0, \quad f_{U}^{\prime \prime}(0)=\sum_{k=1}^{r+s} d_{k}\left[Y_{k}\left\{\frac{u_{k}}{\left|u_{k}\right|}\right\}+X_{k}^{2}\left\{\frac{u_{k}}{\left|u_{k}\right|}\right\}-\left(X_{k}\left\{\frac{u_{k}}{\left|u_{k}\right|}\right\}\right)^{2}\right] .
$$

Si $Z$ est hermitienne et si $v \in \mathbb{C}^{n}$, on a $(Z\{v\})^{2} \leq Z^{2}\{v\}$ avec égalité si et seulement si $v$ est vecteur propre de $Z$ (voir (2.23)). Soit $T_{0}$ l'ensemble des $U \in T$ tels que pour tout $k=1, \ldots, r+s, u_{k}$ est vecteur propre de $X_{k}$. Alors

$$
f_{U}^{(3)}(0)=0, \quad f_{U}^{(4)}(0)=3 \sum_{k=1}^{r+s} d_{k}\left[Y_{k}^{2}\left\{\frac{u_{k}}{\left|u_{k}\right|}\right\}-\left(Y_{k}\left\{\frac{u_{k}}{\left|u_{k}\right|}\right\}\right)^{2}\right] \quad\left(U \in T_{0}\right) .
$$


Il suffit pour conclure de trouver $Y=\left(Y_{k}\right)_{k} \in T_{I} P_{n, L}$ tel que $Y_{k}\left\{u_{k}\right\}=0$ et $Y_{k} u_{k} \neq 0$ pour tout $k=1, \ldots, r+s$ et tout $U=\left(u_{k}\right)_{k} \in T_{0}$. En effet si $Y$ est assez petit, on aura $f_{U}^{\prime \prime}(0)>0$ pour $U \in T \backslash T_{0}$ et $f_{U}^{\prime \prime}(0)=f_{U}^{(3)}(0)=0$, $f_{U}^{(4)}(0)>0$ pour $U \in T_{0}$, d'où la condition $(C)$.

Pour $k$ fixé, notons $E_{k}^{1}, \ldots, E_{k}^{m_{k}}$ les sous-espaces propres de $X_{k}$ (qui contiennent les $u_{k}$ pour tout $\left.U=\left(u_{k}\right)_{k} \in T_{0}\right)$ et $e_{1}, \ldots, e_{n}$ une base orthogonale de vecteurs propres de $X_{k}$. Remarquer que $m_{k} \geq 2$ car $\operatorname{Tr} X_{k}=0$. Étant donnée une forme linéaire $\alpha_{j} \in\left(E_{k}^{j}\right)^{*}$ non nulle sur les $u_{k} \in E_{k}^{j}$ $\left(j=1, \ldots, m_{k}-1\right)$, on pose $Y_{k} x=\alpha_{j}(x) e_{n}$ pour $x \in E_{k}^{j}$ et $1 \leq j<m_{k}$ (il faut bien sûr compléter simultanément $Y_{k}$ pour la rendre hermitienne). Si $d=\operatorname{dim} E_{k}^{m_{k}}=1$, alors $Y_{k}$ convient. Si $d \geq 2$, on considère $F=e_{n}^{\perp} \cap E_{k}^{m_{k}}$ et on prend $\beta \in F^{*}$ non nulle sur les $u_{k} \in F$ (s'il en existe). On termine en posant $Y_{k} x=\beta(x) e_{n-d}(x \in F)$, puis en complétant par symétrie hermitienne.

Soit $\epsilon>0$. On sait d'après [25, prop. 2] que l'ensemble $\left[\epsilon, \infty\left[\cap \mu_{L}\left(P_{n, L}\right)\right.\right.$ est recouvert par l'image d'un compact de $\mathrm{GL}_{n}\left(\mathcal{O}_{L}\right) \backslash G^{r, s}$, donc (ce qui est équivalent) par l'image d'un compact de $\mathrm{GL}_{n}\left(\mathcal{O}_{L}\right) \backslash P_{n, L}$. On peut formuler un résultat plus précis (comparer avec [25]).

Proposition 2.23 («critère de Mahler » pour les formes de Humbert)

L'inégalité $\mu_{L} \geq \epsilon>0$ définit un ensemble compact de $\mathrm{GL}_{n}\left(\mathcal{O}_{L}\right) \backslash P_{n, L}$.

Preuve. - D'après la réduction de Humbert (voir [25, p. 15]), toute $A \in P_{n, L}$ est $\mathrm{GL}_{n}\left(\mathcal{O}_{L}\right)$-équivalente à une forme $N \cdot D$ avec $N=\left(N_{k}\right)_{k}$ où $N_{k}$ est unipotente triangulaire supérieure, $D=\left(D_{k}\right)_{k}$ où $D_{k}=\left(\delta_{i}^{j} t_{k}^{j}\right)_{i, j}$ est diagonale $\left(t_{k}^{j}\right.$ réels $\left.>0\right)$, les modules des coefficients des $N_{k}$ et les rapports $t_{k}^{j} / t_{k}^{j+1}$ $(1 \leq k \leq r+s$ et $1 \leq j \leq n-1)$ étant majorés par une constante ne dépendant que de $L$ et de $n$. Comme $\operatorname{det} D_{k}=1$, on voit que $\left(t_{k}^{1}\right)^{n}=\prod_{j=2}^{n} t_{k}^{1} / t_{k}^{j}$ est majoré par une constante $c$. Par ailleurs soit $u=(1,0, \ldots, 0)^{t}$. Alors $\prod_{k} N_{k} \cdot D_{k}\{u\}=\prod_{k} t_{k}^{1} \geq \epsilon$, d'où $t_{k}^{1} \geq \epsilon c^{-(1-1 / n)}$. Par suite chaque $t_{k}^{j}$ est minoré, donc majoré puisque $\operatorname{det} D_{k}=1$, ce qui achève la preuve.

Nous étudions maintenant la dégénérescence des formes de Humbert pour la métrique riemannienne de $P_{n, L}$. Reprenons les notations de la preuve de la proposition 2.22, 1). Soient $A \in P_{n, L}, X \in T_{A} P_{n, L}$ non nul et $U \in \mathbb{R}^{r} \times \mathbb{C}^{S} \backslash \Delta$. Posons $g_{U}(t)=\ell_{U}\left(A \exp \left(t A^{-1}\right)\right)$. Le hessien de $\ell_{U}$ est donné par $g_{U}^{\prime \prime}(0)$ :

$$
H \ell_{U}(A) \cdot(X, X)=\sum_{k=1}^{r+s} d_{k}\left[A_{k}^{-1}\left\{X_{k} u_{k}\right\} / A_{k}\left\{u_{k}\right\}-\left(X_{k}\left\{u_{k}\right\} / A_{k}\left\{u_{k}\right\}\right)^{2}\right] .
$$

En écrivant $A_{k}=P_{k} P_{k}^{*}$ on voit à partir de l'inégalité $(Z\{v\})^{2} \leq Z^{2}\{v\}$ ( $Z$ hermitienne) que $g_{U}^{\prime \prime}(0) \geq 0$, avec égalité si et seulement si $u_{k}$ est vecteur propre de $A_{k}^{-1} X_{k}$ pour tout $k$ (noter que $A_{k}^{-1} X_{k}$ est diagonalisable avec des sousespaces propres $A_{k}$-orthogonaux). La fonction $\ell_{U}$ est donc convexe. On vérifie 
également que la condition $g_{U}$ constante équivaut à $g_{U}^{\prime}(0)=g_{U}^{\prime \prime}(0)=0$. En particulier, $X$ est une "direction dégénérée » pour $A$ si et seulement s'il existe des scalaires $\lambda_{u}^{k}$ tels que

$$
A_{k}^{-1} X_{k} u^{(k)}=\lambda_{u}^{k} u^{(k)} \quad \text { et } \quad \sum_{k=1}^{r+s} d_{k} \lambda_{u}^{k}=0 \quad\left(1 \leq k \leq r+s, u \in S_{A}\right) .
$$

Par exemple la forme $\mathcal{A}_{1,1}=I$ de $[19](L=\mathbb{Q}(\sqrt{d}), d>0$ et $n=2)$ est dégénérée (et eutactique).

Proposition 2.24. - 1) Soit A une forme de Humbert faiblement eutactique ou non dégénérée. Alors $S_{A}$ engendre $L^{n}$ et on a $\mu_{L}(A) \geq 1$.

2) Modulo $\mathrm{GL}_{n}\left(\mathcal{O}_{L}\right)$, il n'existe qu'un nombre fini de classes minimales de $P_{n, L}$ contenant une forme faiblement eutactique ou non dégénérée.

3) Les formes de Humbert parfaites ou eutactiques non dégénérées sont en nombre fini modulo $\mathrm{GL}_{n}\left(\mathcal{O}_{L}\right)$ et leurs coefficients sont algébriques sur $\mathbb{Q}$.

Preuve. - 1) Observons d'abord que la dimension du sous-espace $M_{k}$ de $\mathbb{R}^{n}$ ou $\mathbb{C}^{n}$ engendré par les conjugués $\left(u^{(k)}\right)_{u \in S_{A}}$ est indépendante de $k$ et vaut le rang de $S_{A}$ dans $L^{n}$. Dans les deux cas considérés, cette dimension est maximale. En effet si $A$ est faiblement eutactique, la forme $A_{k}^{-1}$ (hermitienne $>0$ ) est combinaison linéaire des $\left(u^{(k)} u^{(k) *}\right)_{u \in S_{A}}$ (voir (2.24)), ce qui implique $M_{k}^{\perp}=\{0\}$. D'autre part, si $\operatorname{dim} M_{k}=n-p<n$, on choisit des scalaires $\left(\lambda_{k}\right)_{k}$ non nuls tels que $\sum_{k} d_{k} \lambda_{k}=0$, puis on définit $X \in T_{A} P_{n, L}$ par $X_{k} x=\lambda_{k} A_{k} x$ $\left(x \in M_{k}\right)$ et $X_{k} x=(1-n / p) \lambda_{k} A_{k} x\left(x \in M_{k}^{\perp}\right)$. Il est clair que $X$ est une direction dégénérée pour $A$ (voir (2.25)).

La relation $\mu_{L}(A) \geq 1$ résulte de l'inégalité de Hadamard. Soit $u_{1}, \ldots, u_{n}$ un système de rang $n$ extrait de $S_{A}$ et soit $M=\left(u_{1}, \ldots, u_{n}\right)$ la matrice des coordonnées des $u_{j}\left(M \in M_{n}\left(\mathcal{O}_{L}\right)\right.$ et $\left.\operatorname{det} M \neq 0\right)$. Le déterminant de Gram pour $A_{k}$ des conjugués $u_{1}^{(k)}, \ldots, u_{n}^{(k)}$ dans $\mathbb{R}^{n}$ ou $\mathbb{C}^{n}$ vaut

$$
\operatorname{det}\left(M^{(k) *} A_{k} M^{(k)}\right)=\left|(\operatorname{det} M)^{(k)}\right|^{2}
$$

et il est majoré par $\prod_{j=1}^{n} A_{k}\left\{u_{j}^{(k)}\right\}$. Par suite, on peut conclure grâce à l'inégalité

$$
\prod_{k=1}^{r+s}\left|(\operatorname{det} M)^{(k)}\right|^{2 d_{k}} \leq\left[\mu_{L}(A)\right]^{n}
$$

dont le premier membre est un entier naturel non nul.

2) D'après 1 ), toute classe d'équivalence minimale contenant un point faiblement eutactique ou non dégénéré rencontre l'ensemble compact défini par $\mu_{L} \geq 1$ dans $\mathrm{GL}_{n}\left(\mathcal{O}_{L}\right) \backslash P_{n, L}$ (proposition 2.23), d'où la finitude de ces classes.

3) Soit $S$ une partie finie de $\mathcal{O}_{L}^{n} \backslash\{0\}$. Il est clair que le sous-ensemble $\widetilde{\mathcal{C}}_{S}$ de $P_{n, L}$ défini par l'égalité des longueurs $\left(\ell_{\sigma(u)}\right)_{u \in S}$ est algébrique défini sur $\overline{\mathbb{Q}}$. 
Il en est de même pour l'ensemble $\mathcal{R}_{<r}^{S}$ des $A \in P_{n, L}$ pour lesquelles le rang de $\left(\nabla \ell_{\sigma(u)}(A)\right)_{u \in S}$ est inférieur ou égal à $r$ (voir (2.24)). On a donc l'algébricité des formes parfaites ou eutactiques non dégénérées (proposition 1.8, 1.9 et lemme 1.11). Les assertions de finitude résultent de 2) car dans chaque classe minimale, il y a finitude des formes parfaites (proposition 1.8 et lemme 1.11) et unicité d'une éventuelle forme eutactique non dégénérée (proposition 1.6, assertion 2).

Note 2.19. - Finitude et algébricité des formes de Humbert parfaites sont établies dans $[19, \S 4]$.

2.12. Systole des surfaces de Riemann. - Soit $\Sigma_{g}$ la surface topologique orientable fermée de genre $g \geq 2$ et soit $\mathcal{C}_{g}$ l'ensemble des classes de conjugaison non triviales de son groupe fondamental $\Pi_{1}\left(\Sigma_{g}\right)$. L'espace de Teichmüller $\mathcal{T}_{g}$ associé est muni des fonctions longueur géodésiques $\left(\ell_{\gamma}\right)_{\gamma \in \mathcal{C}_{q}}$ qui sont analytiques. Localement sur $\mathcal{T}_{g}$, il n'y a qu'un nombre fini de $\ell_{\gamma}$ pouvant prendre une valeur petite (voir [2]). La systole $\sigma(X)$ de $X \in \mathcal{T}_{g}$ est par définition la longueur minimale d'une géodésique fermée. C'est une fonction invariante sous l'action du groupe modulaire de Teichmüller $\operatorname{Mod}_{g}$ et on a les notions de surface de Riemann parfaite, eutactique,... pour $\sigma$ (définition 1.2). Comme les $\ell_{\gamma}$ sont strictement convexes pour la métrique de Weil-Petersson (voir [38]), tous les points sont non dégénérés et on a automatiquement un théorème de Voronoï. En outre toute classe minimale contient au plus une surface semi-eutactique, par exemple extrême (proposition 1.6, 1). Une première caractérisation des surfaces de Riemann extrêmes ainsi que la finitude de ces surfaces modulo $\operatorname{Mod}_{g}$ ont été établies dans [33] (voir aussi [5, § 2.3]). En fait les surfaces parfaites ou semi-eutactiques sont déjà en nombre fini (théorème 6,2 ).

La forme d'intersection sur $H_{1}\left(\Sigma_{g}, \mathbb{Z}\right)$ nous permet de définir une notion d'isotropie : $X \in \mathcal{T}_{g}$ sera dite isotrope si ses systoles (géodésiques fermées de longueur minimale) engendrent un sous-espace totalement isotrope pour l'intersection, i.e. sont disjointes deux à deux (rappelons que deux systoles ont au plus un point commun). Par le lemme du collier (voir [15]), la systole d'une surface non isotrope admet la minoration $\sinh \left(\frac{1}{2} \sigma(X)\right) \geq 1$. On en déduit par compacité (voir la preuve du théorème 6,2$)$ que l'ensemble des classes minimales non isotropes est fini modulo $\operatorname{Mod}_{g}$.

ThÉORÈme 6 (finitude et «algébricité » des surfaces parfaites ou eutactiques)

1) Toute surface de Riemann faiblement eutactique est non isotrope.

2) Modulo l'action de $\operatorname{Mod}_{g}$, il n'existe dans $\mathcal{T}_{g}$ qu'un nombre fini de surfaces de Riemann parfaites ou semi-eutactiques pour la systole.

3) Si $X \in \mathcal{T}_{g}$ est parfaite ou eutactique, alors les longueurs des géodésiques fermées de $X$ (et en particulier sa systole) sont des logarithmes de nombres algébriques sur $\mathbb{Q}$.

BULlETIN DE LA SOCIÉTÉ MATHÉMATIQUE DE FRANCE 
Preuve. - 1) Pour $X$ parfaite, on a mieux : toute systole doit couper une autre systole. Sinon on pourrait déformer $X$ (par un «twist » de Fenchel-Nielsen) dans sa classe minimale en contradiction avec la proposition 1.8. Plus généralement soit $X$ faiblement eutactique et isotrope de systole $\sigma_{0}$. En incluant l'ensemble $S(X)$ des systoles de $X$ dans un système maximal de courbes simples fermées, on peut découper $X$ en pantalons et obtenir une variation $X_{t}$ de $X=X_{0}$ telle que $\ell_{\gamma}\left(X_{t}\right)=\exp (t) \sigma_{0}$ pour tout $\gamma \in S(X)$. Cela contredit le lemme 1.1,4).

2) On a ici un analogue du critère de compacité de Mahler : pour tout $\alpha>0$ l'ensemble des surfaces $X$ telles que $\sigma(X) \geq \alpha$ est compact dans l'espace des modules $\operatorname{Mod}_{g} \backslash \mathcal{T}_{g}$ (voir [30]). Par conséquent, modulo l'action de $\operatorname{Mod}_{g}$, il n'existe qu'un nombre fini de classes minimales qui rencontrent l'ensemble $\sigma \geq \alpha$ dans $\mathcal{T}_{g}$. D'où la finitude des classes non isotropes, et celle des surfaces semi-eutactiques (proposition 1.6, 1), unicité). La finitude des surfaces parfaites découlera des propriétés algébriques établies au 3 ).

3) Rappelons que $\mathcal{T}_{g}$ est aussi l'espace des représentations $\rho$ de $\Pi_{1}\left(\Sigma_{g}\right)$ dans $\mathrm{SL}_{2}(\mathbb{R})$ considérées à conjugaison près. Longueurs géodésiques et traces sont reliées par la formule

$$
4 \cosh ^{2}\left(\frac{1}{2} \ell_{\gamma}(\rho)\right)=\operatorname{Tr}^{2} \rho(\gamma) \quad\left(\gamma \in \mathcal{C}_{g}\right) .
$$

On sait qu'il existe $\gamma_{1}, \ldots, \gamma_{N}(N=N(g))$ tels que les traces associées donnent un homéomorphisme de $\mathcal{T}_{g}$ sur une composante connexe d'une sous-variété algébrique réelle de $\mathbb{R}^{N}$ définie par des équations à coefficients entiers (voir [23] et [36]), et tels que chaque trace $\operatorname{Tr} \rho(\gamma)$ ( $\gamma$ fixé) soit un polynôme à coefficients entiers en les coordonnées de $\mathbb{R}^{N}$, i.e. les traces « de base» (voir aussi [24]). Il est clair que la nature des points particuliers (parfaits, eutactiques,...) est inchangée quand on compose au but les fonctions longueurs par un même $\mathbb{C}^{1}$ difféomorphisme croissant. On peut donc prendre les $t_{\gamma}(\rho)=\operatorname{Tr}^{2} \rho(\gamma)\left(\gamma \in \mathcal{C}_{g}\right)$ comme fonctions longueur. Les relations d'égalité entre les $t_{\gamma}$ correspondent à des sous-ensembles algébriques de $\mathbb{R}^{N}$ définis sur $\mathbb{Z}$; par suite, si $S$ est une partie finie de $\mathcal{C}_{g}$, la classe minimale $\mathcal{C}_{S}$ ne contient qu'un nombre fini de surfaces parfaites et leurs coordonnées sont algébriques (proposition 1.8 et lemme 1.11). La finitude des surfaces parfaites $\left(\operatorname{modulo} \operatorname{Mod}_{g}\right)$ résulte alors de celle des classes minimales non isotropes. Enfin, en procédant comme dans la preuve du corollaire 1.12, 2), on voit que la condition $\operatorname{rang}\left(\mathrm{d} t_{\gamma} \mid \mathcal{T}_{g}\right)_{\gamma \in S} \leq r$ est donnée par des équations polynomiales à coefficients entiers. Par conséquent (proposition 1.9 et lemme 1.11), les surfaces eutactiques sont à coordonnées algébriques.

Remarques 2.20. - 1) Les assertions 1) et 3) du théorème 6 sont valables avec la même preuve pour $\sigma^{\mathcal{H}}=\inf _{\gamma \in \mathcal{H}} \ell_{\gamma}$ où $\mathcal{H}$ est une partie non vide de $\mathcal{C}_{g}$ invariante par $\operatorname{Mod}_{g}$, par exemple pour la systole homologique ( $\mathcal{H}$ est l'ensemble des $\gamma \in \mathcal{C}_{g}$ non nulles en homologie). 
2) Il est montré dans [2] que la systole est une fonction de Morse topologique sur $\mathcal{T}_{g}$ dont les points critiques sont les surfaces eutactiques, ce qui permet aussi d'établir la finitude de ces surfaces (voir $[2, \S 6.3]$ ).

On ne connaît qu'un petit nombre de surfaces de Riemann fermées parfaites (voir [16, 21, 33, 34, 35]). En revanche, il est plus facile de trouver des surfaces eutactiques. Par exemple toute surface «triangulaire» (i.e. dont le groupe d'automorphismes est le quotient d'un groupe triangulaire de réflexions hyperboliques) est eutactique comme point fixe isolé d'un sous-groupe de $\operatorname{Mod}_{g}$ (corollaire 1.3). D'autres surfaces eutactiques sont données dans [17].

\section{BIBLIOGRAPHIE}

[1] Akrout (H.) - Théorème de Voronoï dans les espaces symétriques, Canad. J. Math., t. 54 (2002), no. 3, pp. 449-467.

[2] _ Singularités topologiques des systoles généralisées, Topology, t. 42 (2003), pp. 291-308.

[3] Baeza (R.) \& ICAZA (M.I.) - On Humbert-Minkowski's constant for a number field, Proc. Amer. Math. Soc., t. 125 (1997), no. 11, pp. 3195-3202.

[4] BAVARD (C.) - Invariant d'Hermite isotrope et densité des réseaux orthogonaux lorentziens, juin 2003.

[5] _ Systole et invariant d'Hermite, J. reine angew. Math., t. 482 (1997), pp. 93-120.

[6] _ Familles hyperboliques de réseaux symplectiques, Math. Ann., t. 320 (2001), pp. 799-833.

[7] Bergé (A.-M.) - Minimal vectors of pairs of dual lattices, J. Number Theory, t. 52 (1995), no. 2, pp. 284-298.

[8] Bergé (A.-M.) \& Martinet (J.) - Sur un problème de dualité lié aux sphères en géométrie des nombres, J. Number Theory, t. 32 (1989), no. 1, pp. $14-42$.

[9] _ Réseaux extrêmes pour un groupe d'automorphismes, in Journées Arithmétiques (Luminy, 1989), Astérisque, vol.198-200, Société Mathématique de France, 1991, pp. 41-66.

[10] _ Densité dans des familles de réseaux. Application aux réseaux isoduaux, Enseign. Math., t. 41 (1995), no. 3-4, pp. 335-365.

[11] _ Sur la classification des réseaux eutactiques, J. London Math. Soc., t. 53 (1996), no. 3, pp. 417-432.

[12] Bergé (A.-M.), Martinet (J.) \& Sigrist (F.) - Une généralisation de l'algorithme de Voronoï pour les formes quadratiques, in Journées Arithmétiques (Geneva, 1991), Astérisque, vol. 209, Société Mathématique de France, 1992, pp. 12, 137-158. 
[13] Bochnak (J.), Coste (M.) \& Roy (M.-F.) - Géométrie algébrique réelle, Ergebnisse der Mathematik und ihrer Grenzgebiete, vol.12, Springer-Verlag, Berlin, 1987.

[14] Bourbaki (N.) - Éléments de mathématique. Livre II : Algèbre. Chapitre 8 : Modules et anneaux semi-simples, Actualités Scientifiques et Industrielles, vol. 1261, Hermann, 1958.

[15] Buser (P.) - The collar theorem and examples, Manuscripta Math., t. 25 (1978), no. 4, pp. 349-357.

[16] Casamayou (A.) - Surfaces de Riemann parfaites en genre 4 et 6, Comment. Math. Helv., à paraître.

[17] _ Surfaces de Riemann parfaites en petit genre, Thèse, Université Bordeaux I, juillet 2000.

[18] Coulangeon (R.) - Réseaux k-extrêmes, Proc. London Math. Soc., t. 73 (1996), no. 3, pp. 555-574.

[19] _ Voronoï theory over algebraic number fields, Monogr. Enseign. Math., t. 37 (2001), pp. 147-162.

[20] EBerLein (P.) - Structure of manifolds of nonpositive curvature, in Global differential geometry and global analysis 1984 (Berlin, 1984), Lect. Notes in Math., vol. 1156, Springer, 1985, pp. 86-153.

[21] Hamenstädt (U.) - New examples of maximal surfaces, Enseign. Math., t. 47 (2001), no. 1-2, pp. 65-101.

[22] Helgason (S.) - Differential geometry, Lie groups, and symmetric spaces, Academic Press, New York, 1978.

[23] Helling (H.) - Diskrete Untergruppen von $\mathrm{SL}_{2}(\mathbb{R})$, Invent. Math., t. 17 (1972), pp. 217-229.

[24] Honowitz (R.D.) - Characters of free groups represented in the twodimensional special linear group, Comm. Pure Appl. Math., t. 25 (1972), pp. 635-649.

[25] ICAZA (M.I.) - Hermite constant and extreme forms for algebraic number fields, J. London Math. Soc., t. 55 (1997), no. 1, pp. 11-22.

[26] Jaquet-Chiffelle (D.-O.) - Trois théorèmes de finitude pour les Gformes, J. Théor. Nombres Bordeaux, t. 7 (1995), no. 1, pp. 165-176.

[27] Korkine (A.) \& ZolotarefF (G.) - Sur les formes quadratiques positives, Math. Ann., t. 11 (1877), pp. 242-292.

[28] Martinet (J.) - Les réseaux parfaits des espaces euclidiens, Masson, Paris, 1996.

[29] Milnor (J.) \& Husemoller (D.) - Symmetric bilinear forms, Ergebnisse der Mathematik und ihrer Grenzgebiete, vol.73, Springer-Verlag, New York, 1973.

[30] Mumford (D.) - A remark on Mahler's compactness theorem, Proc. Amer. Math. Soc., t. 28 (1971), no. 1, pp. 289-294. 
[31] Raghunathan (M.S.) - Discrete subgroups of Lie groups, Ergebnisse der Mathematik und ihrer Grenzgebiete, vol. 68, Springer-Verlag, New York, 1972.

[32] Rankin (R.A.) - On positive definite quadratic forms, J. London Math. Soc., t. 28 (1953), pp. 309-314.

[33] Schmutz (P.) - Riemann surfaces with shortest geodesic of maximal length, Geom. Funct. Anal., t. 3 (1993), no. 6, pp. 564-631.

[34] _ Systoles on Riemann surfaces, Manuscripta Math., t. 85 (1994), no. 3-4, pp. 429-447.

[35] Schmutz Schaller (P.) - Perfect non-extremal Riemann surfaces, Canad. Math. Bull., t. 43 (2000), no. 1, pp. 115-125.

[36] Seppälä (M.) \& Sorvali (T.) - Affine coordinates for Teichmüller spaces, Math. Ann., t. 284 (1989), no. 1, pp. 165-176.

[37] Voronoï (G.) - Nouvelles applications des paramètres continus à la théorie des formes quadratiques, premier mémoire. Sur quelques propriétés des formes quadratiques positives parfaites, J. reine angew. Math., t. 133 (1908), pp. 97-178.

[38] Wolpert (S.A.) - Geodesic length functions and the Nielsen problem, J. Differential Geom., t. 25 (1987), no. 2, pp. 275-296. 